\title{
A 3D cell-centered ADER MOOD Finite Volume method for solving updated Lagrangian hyperelasticity on unstructured grids
}

\author{
Walter Boscheri ${ }^{\mathrm{a}}$, Raphaël Loubère ${ }^{\mathrm{b}}$, Pierre-Henri Maire ${ }^{\mathrm{c}}$. \\ ${ }^{a}$ Dipartimento di Matematica e Informatica, Ferrara Italy \\ ${ }^{\mathrm{b}}$ Institut de Mathématiques de Bordeaux (IMB), Talence, France \\ ${ }^{\mathrm{c}}$ CEA-CESTA, Le Barp, France
}

\begin{abstract}
In this paper, we present a conservative cell-centered Lagrangian Finite Volume scheme for solving the hyperelasticity equations on unstructured multidimensional grids. The starting point of the present approach is the cell-centered FV discretization named EUCCLHYD and introduced in the context of Lagrangian hydrodynamics. Here, it is combined with the a posteriori Multidimensional Optimal Order Detection (MOOD) limiting strategy to ensure robustness and stability at shock waves with piecewise linear spatial reconstruction. The ADER (Arbitrary high order schemes using DERivatives) approach is adopted to obtain second-order of accuracy in time. This strategy has been successfully tested in an hydrodynamics context and the present work aims at extending it to the case of hyperelasticity. Here, the hyperelasticty equations are written in the updated Lagrangian framework and the dedicated Lagrangian numerical scheme is derived in terms of nodal solver, Geometrical Conservation Law (GCL) compliance, subcell forces and compatible discretization. The Lagrangian numerical method is implemented in 3D under MPI parallelization framework allowing to handle genuinely large meshes. A relatively large set of numerical test cases is presented to assess the ability of the method to achieve effective second order of accuracy on smooth flows, maintaining an essentially non-oscillatory behavior and general robustness across discontinuities and ensuring at least physical admissibility of the solution where appropriate. Pure elastic neo-Hookean and non-linear materials are considered for our benchmark test problems in $2 \mathrm{D}$ and 3D. These test cases feature material bending, impact, compression, non-linear deformation and further bouncing/detaching motions.
\end{abstract}

Key words: Cell-centered Lagrangian finite volume schemes, moving unstructured 
meshes, a posteriori MOOD limiting, ADER, hyper-elasticity

\section{Contents}

1 Introduction 2

2 Updated Lagrangian hyperelastic modeling for isotropic materials 4

2.1 Kinematics 5

2.2 Governing equations 8

2.3 Updated Lagrangian hyperelasticity for isotropic materials 13

3 Finite volume discretization $\quad 14$

3.1 Mesh and notation 14

3.2 Discrete velocity gradient operators 16

$\begin{array}{lll}3.3 & \text { Semi-discretization in space } & 17\end{array}$

3.4 Space-Time discretization - ADER methodology 20

3.5 Limiting: a posteriori MOOD loop $\quad 27$

3.6 Internal consistency consideration 28

3.7 Time-step monitoring 29

3.8 Boundary condition treatments 29

4 Implementation considerations 30

4.1 Algorithm 30

4.2 Meshing and parallelization 31

$5 \quad 2 \mathrm{D}$ and $3 \mathrm{D}$ test problems 31

5.1 Swinging plate $\quad 32$

5.2 Elastic vibration of a Beryllium plate 33

5.3 Finite deformation of a cantilever thick beam 35

5.4 Blake's problem 37

5.5 Twisting column 38

5.6 Rebound of a hollow circular bar $\quad 39$

5.7 Impact of a jelly-like droplet $\quad 42$

5.8 L-shaped block $\quad 43$

5.9 Monitoring of the internal consistency 45

6 Conclusions and perspectives $\quad 47$

A Principal invariants of a tensor $\quad 48$

B Boundary conditions (BCs) 49

References $\quad 50$

\section{Introduction}

This work is concerned with the accurate multi-dimensional simulation of hyper-elasticity models by updated Lagrangian Finite Volume (FV) numerical scheme.

Traditionally displacement-based Finite Element (FE) frameworks are employed when simulating engineering large strain transient situations, see for instance $[34,27,6]$. Tetrahedral

Email addresses: walter.boscheri@unife.it (Walter Boscheri), raphael.loubere@u-bordeaux.fr (Raphaël Loubère), pierre-henri.maire@cea.fr (Pierre-Henri Maire). 
mesh elements are often preferred for the discretization because the mesh generators are easily available. Nonetheless, such FE approach presents some well reported issues, for instance a reduced order of convergence for strains and stresses [48], poor performance in nearly incompressible bending dominated scenarios [40] and spurious numerical instabilities (shear or volumetric locking, parasitical hydrostatic pressure fluctuations, etc.) [5], reduction of the accuracy when artificial stabilization terms are added. Notice that the introduction of high order schemes usually helps but does not cure all of these issues.

The earliest attempt at solving solid dynamics equations in a FV framework is to be found in the papers $[56,55]$. In this seminal approach, the solid dynamics equations are written under Eulerian formalism as a first-order system of conservation laws. Their FV discretization relies on Godunov-type methods.

In a series of papers $[1,8,33,9]$ an original and promising FV computational framework has been constructed for solving solid dynamics equations written under total Lagrangian formulation. Impressive three-dimensional simulations of hyperelastic materials undergoing large deformations are achieved within this framework. The underlying numerical fluxes are obtained employing an acoustic approximate Riemann solver. Further, the total Lagrangian FV discretization of the physical conservation laws relies on the discretization of the time rate of change of the deformation gradient, its determinant and its co-factor. Here, the deformation gradient is nothing but the Lagrange-Euler mapping which relates the initial and the current configuration of the material under consideration. Let us point out that these three supplementary geometrical conservation laws are redundant and nothing in this numerical method guarantees their consistency at the discrete level.

The development of cell-centered FV Lagrangian schemes based on nodal solvers $[23,42,22]$ for gas dynamics has been extended to 2D and 3D updated Lagrangian hyperelasticity $[39,14]$. Others have explored the used of a mixed velocity-pressure approach using a Variational Multi-Scale method [53], possibly with stabilized nodal stresses [19].

This work also considers the updated Lagrangian framework like for $[39,14]$ contrarily to $[8,33,9]$. Previously in [13] we have presented a second-order accurate cell-centered Lagrangian scheme on unstructured mesh restricted to the hydrodynamics system of conservation laws. This scheme is constructed upon a subcell discretization, popularized for staggered Lagrangian schemes [15,16] and later extended to cell-centered ones [45], further associated with a nodal solver relying on total energy conservation and Geometrical Conservation Law (GCL) compliance. Second-order of accuracy is usually gained by a MUSCL-like approach - piecewise linear reconstructions supplemented with limiters - and a predictor-corrector, Runge-Kutta or a Generalized Riemann Problem (GRP) time discretization.

Contrarily, for the scheme in [13], we have relied on ADER (Arbitrary high order schemes using DERivatives) methodology developed originally from an Eulerian perspective [54,10]. This approach is further supplemented with an a posteriori MOOD limiting strategy [21] to stabilize and produce a fail-safe Lagrangian scheme. We have shown in [13] that such a cellcentered numerical method is able to perform on classical and demanding hydrodynamics test cases using unstructured mesh made of simplexes in 2D and 3D.

In this work we propose the extension of this numerical method to solve problems involving 2D and 3D elastic materials. We ought to solve an hyperelasticity model of PDEs (Partial 
Differential Equations) $[39,12,40,14]$. Historically hypoelasticity models $[57,7,58]$ have been sometimes preferred, see for instance $[59,30,47,52,20]$. A parallel discussion about hypo- and hyper-elastic models and their resolution can be found for instance in [49]. Here, we are interested in simulating the large deformations undergone by isotropic hyperelastic materials. The set of conservation laws under consideration is written under updated Lagrangian representation and equipped with a constitutive law which satisfies not only the principle of material frame indifference but is also thermodynamically consistent [35]. In this approach, the Cauchy stress tensor is the derivative of the free energy with respect to the left CauchyGreen tensor which is a relevant measure of deformation. Moreover, one can show that the Cauchy stress tensor might be expressed uniquely in terms of this deformation tensor. Taking advantage of the simplicial grid, we introduce a specific discretization of the time rate of change of the left Cauchy Green tensor to ensure its compatibility with the Geometric Conservation Law, refer to [28]. In this article are tackled several issues related to the 3D extension of our ADER Lagrangian scheme, as well as the increase of complexity in the modeling of hyper-elastic materials. First, the hyperelastic model demands the resolution of a constitutive law which, in the framework of ADER methodology, requires some adaptation. Second, the a posteriori MOOD limiting strategy must consider new admissibility criteria brought by the model in order to still ensure the robust and fail-safe characteristics while maintaining an acceptable, if not optimal, accuracy. Third, the boundary conditions (BCs) must be dealt with care as materials may balistically fly but also impact, bounce, slide, spread, tear apart onto a wall or different materials. Fourth, in relation to the points

above, 3D Lagrangian numerical code requires extra-care as efficient simulations demand a well designed parallelization methodology as well as appropriate BCs and robust limiting strategy.

Numerical results involving materials enduring large deformation (bending, twisting, etc.) adopted from $[40,36,14]$ will be presented to assess the ability of this updated Lagrangian numerical scheme to simulate such hyperelastic situations.

For a broad and modern introductions on hypo- or hyper-elasticity we refer the readers in particular to $[39,12,14,49]$. For 3D Lagrange updated FV numerical schemes among many works we refer to $[17,43,31,14]$. In the forthcoming section the paper presents in details the hyperelastic model and the governing equations to be solved. Then in the third section, the Lagrangian numerical scheme is introduced with emphasis on the ADER approach, the nodal solver, the a posteriori limiting strategy and some discussion on the internal consistency, boundary conditions and implementation considerations. All numerical tests are gathered in the fourth section. We propose the numerical results of our simulations for a large set of $2 \mathrm{D}$ and 3D problems involving elastic materials impacting, detaching, compressing, swinging, twisting, etc. Conclusions and perspectives are finally drawn in the last section.

\section{Updated Lagrangian hyperelastic modeling for isotropic materials}

In this section, we aim at recalling the conservation laws describing the time evolution of isotropic solid materials undergoing large deformations. The resulting conservation laws of mass, momentum and total energy shall be written under the updated Lagrangian form. The isotropic materials under consideration are characterized by an hyperelastic constitutive law. 
Namely, the Cauchy stress tensor is defined as being the derivative of the free energy with respect to the deformation tensor. In this framework, the material frame indifference principle and the thermodynamic consistency are automatically satisfied. The interested reader might refer for instance to [35] for further developments on these subtle topics. For the sake of completeness, we recall hereafter some notions of kinematics that shall be useful for writing the conservation and constitutive laws.

\subsection{Kinematics}

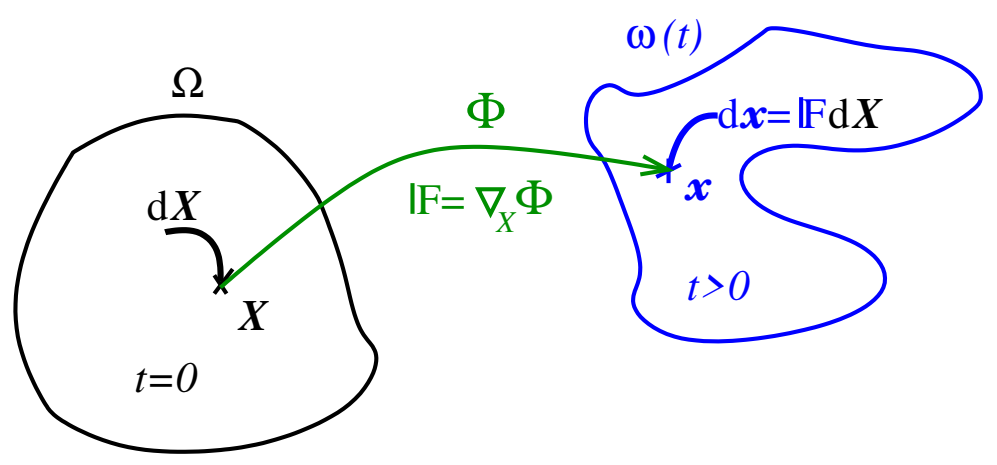

Fig. 1. Sketch of the Lagrangian-Eulerian mapping relating a material Lagrangian point $\boldsymbol{X}$ at $t=0$ and a spatial Eulerian one $\boldsymbol{x}$ at $t>0$ through $\boldsymbol{\Phi}$, and its Jacobian $\mathbb{F}(\boldsymbol{X}, t)=\frac{\partial \boldsymbol{\Phi}}{\partial \boldsymbol{X}}(\boldsymbol{X}, t)$.

\subsubsection{Lagrange-Euler mapping.}

We consider a solid body in the three-dimensional Euclidean space occupying the region $\Omega$ in its initial configuration at time $t=0$, and the region $\omega(t)$ in its current configuration at time $t>0$. The motion of this body is characterized by the smooth function, $\boldsymbol{\Phi}$, that assigns to each material point $\boldsymbol{X}$ and time $t$ the spatial point $\boldsymbol{x}$ such that

$$
\begin{aligned}
& \Omega \longrightarrow \omega(t) \\
& \boldsymbol{X} \longmapsto \boldsymbol{x}=\boldsymbol{\Phi}(\boldsymbol{X}, t) .
\end{aligned}
$$

This smooth function is the Lagrange-Euler mapping which relates the Lagrangian (material) point $\boldsymbol{X}$ to its Eulerian (spatial) counterpart $\boldsymbol{x}$. By definition, this mapping satisfies $\boldsymbol{\Phi}(\boldsymbol{X}, 0)=\boldsymbol{X}$ and its Jacobian, also named the deformation gradient, reads

$$
\mathbb{F}(\boldsymbol{X}, t)=\nabla_{X} \boldsymbol{\Phi}(\boldsymbol{X}, t)
$$

where the symbol $\nabla_{X}$ denotes the gradient operator with respect to the Lagrangian coordinate. The determinant of the deformation gradient is denoted $J(\boldsymbol{X}, t)=\operatorname{det}(\mathbb{F}(\boldsymbol{X}, t))$ and satisfies $J(\boldsymbol{X}, t=0)=1$ since $\mathbb{F}(\boldsymbol{X}, t=0)=\mathbb{I}_{\mathrm{d}}$ where $\mathbb{I}_{\mathrm{d}}$ is the identity tensor. A continuity argument leads us to assume that $J(\boldsymbol{X}, t)>0$ for all $t>0$, ensuring as such that $\boldsymbol{\Phi}$ is a one-to-one mapping.

A physical quantity can be expressed both in terms of the Lagrangian coordinate and in terms of the Eulerian one. More precisely, let $G(\boldsymbol{X}, t)$ denotes the Lagrangian representation of a physical quantity. Its Eulerian representation reads $g(\boldsymbol{x}, t)$. Obviously, these are two 
representations of the same physical quantity and, as a consequence, they fulfill the identities

$$
g(\boldsymbol{x}, t)=G\left[\boldsymbol{\Phi}^{-1}(\boldsymbol{x}, t), t\right], \quad \text { and }, \quad G(\boldsymbol{X}, t)=g[\boldsymbol{\Phi}(\boldsymbol{X}, t), t] .
$$

In what follows, the same notation shall be employed for both descriptions.

Time differentiating the mapping, holding $\boldsymbol{X}$ fixed, allows us to define the kinematic velocity

$$
\boldsymbol{v}(\boldsymbol{X}, t)=\left.\frac{\partial \boldsymbol{\Phi}}{\partial t}\right|_{\boldsymbol{X}}(\boldsymbol{X}, t)
$$

Now, time differentiating the identity $g(\boldsymbol{X}, t)=g(\boldsymbol{\Phi}(\boldsymbol{X}, t))$, holding $\boldsymbol{X}$ fixed and applying the chain rule, yields

$$
\left.\frac{\partial g}{\partial t}\right|_{\boldsymbol{X}}(\boldsymbol{X}, t)=\left.\frac{\partial g}{\partial t}\right|_{\boldsymbol{x}}(\boldsymbol{x}, t)+\boldsymbol{v}(\boldsymbol{X}, t) \cdot \nabla_{x} g
$$

where $\nabla_{x} g$ is the gradient of $g$ with respect to the Eulerian coordinate, i.e., $\nabla_{x} g=\frac{\partial g}{\partial x}$. Thus, the Lagrangian time derivative is nothing but the material time derivative which writes

$$
\frac{\mathrm{d} g}{\mathrm{~d} t}(\boldsymbol{x}, t)=\frac{\partial g}{\partial t}(\boldsymbol{x}, t)+\boldsymbol{v} \cdot \nabla_{x} g
$$

\subsubsection{Measures of deformation}

Let us consider the infinitesimal material fiber $\mathrm{d} \boldsymbol{X}$ in the initial configuration which maps into $\mathrm{d} \boldsymbol{x}=\mathbb{F} \mathrm{d} \boldsymbol{X}$ through the motion. We express the stretching of this infinitesimal fiber as follows

$$
\mathrm{d} \boldsymbol{x} \cdot \mathrm{d} \boldsymbol{x}-\mathrm{d} \boldsymbol{X} \cdot \mathrm{d} \boldsymbol{X}=\left(\mathbb{C}-\mathbb{I}_{\mathrm{d}}\right) \mathrm{d} \boldsymbol{x} \cdot \mathrm{d} \boldsymbol{x},
$$

where $\mathbb{C}=\mathbb{F}^{t} \mathbb{F}$ is the right Cauchy-Green tensor. On the other hand, noticing that $\mathrm{d} \boldsymbol{X}=$ $\mathbb{F}^{-1} \mathrm{~d} \boldsymbol{x}$, we also express the stretching of the infinitesimal fiber as follows

$$
\mathrm{d} \boldsymbol{x} \cdot \mathrm{d} \boldsymbol{x}-\mathrm{d} \boldsymbol{X} \cdot \mathrm{d} \boldsymbol{X}=\left(\mathbb{I}_{\mathrm{d}}-\mathbb{B}^{-1}\right) \mathrm{d} \boldsymbol{X} \cdot \mathrm{d} \boldsymbol{X}
$$

where $\mathbb{B}=\mathbb{F F}^{t}$ is the left Cauchy-Green tensor. The right and the left Cauchy-Green tensors are symmetric positive definite and share the same eigenvalues, refer to [35]. These tensors are relevant measures of deformation since for a rigid rotation they boil down to the identity tensor.

\subsubsection{Geometric conservation law (GCL)}

Time differentiating the deformation gradient, $\mathbb{F}=\nabla_{X} \boldsymbol{\Phi}$, recalling that the partial time derivative of the mapping is the kinematic velocity $\boldsymbol{v}=\frac{\partial \Phi}{\partial t}$, leads to the Geometric Conservation Law (GCL) written under total Lagrangian form as

$$
\frac{\partial \mathbb{F}}{\partial t}-\nabla_{X} \boldsymbol{v}=0
$$

where $\mathbb{F}(\boldsymbol{X}, 0)=\mathbb{I}_{\mathrm{d}}$. The GCL is supplemented with the compatibility constraint $\nabla_{X} \times \mathbb{F}=\mathbf{0}$, which ensures that the solution of the foregoing partial differential equation corresponds to the gradient of a mapping. Here, for any second order tensor $\mathbb{T}, \nabla_{X} \times \mathbb{T}$ denotes the curl of $\mathbb{T}$. It is the tensor defined by $\left(\nabla_{X} \times \mathbb{T}\right) \boldsymbol{a}=\nabla_{X} \times\left(\mathbb{T}^{t} \boldsymbol{a}\right)$ for all constant vector $\boldsymbol{a}$. We 
note that the compatibility constraint is an involution constraint for the GCL. Namely, if this constraint is fulfilled initially, it will be satisfied for all time $t>0$. The satisfaction of this compatibility constraint at the discrete level is the cornerstone which any proper discretization of the conservation laws written in total Lagrangian form should rely on, refer to $[28]$.

Introducing the material time derivative and applying the chain rule, we express the GCL under the updated Lagrangian form

$$
\frac{\mathrm{d} \mathbb{F}}{\mathrm{d} t}-\left(\nabla_{x} \boldsymbol{v}\right) \mathbb{F}=0
$$

Here, the deformation gradient and the velocity are viewed as functions of the spatial coordinate $\boldsymbol{x}$. The notation $\mathbb{L}=\nabla_{x} \boldsymbol{v}$ represents the velocity gradient tensor with respect to the current configuration. Employing this notation, the updated Lagrangian form of the GCL reads

$$
\frac{\mathrm{d} \mathbb{F}}{\mathrm{d} t}-\mathbb{L} \mathbb{F}=0 .
$$

Bearing this in mind, let us investigate two important consequences of the GCL that will be useful in the sequel.

The first one is related to the time rate of change of the Jacobian, $J=\operatorname{det} \mathbb{F}$, which is deduced from the GCL thanks to the chain rule

$$
\frac{\mathrm{d}(\operatorname{det} \mathbb{F})}{\mathrm{d} t}=\frac{\partial(\operatorname{det} \mathbb{F})}{\partial \mathbb{F}}: \frac{\mathrm{d} \mathbb{F}}{\mathrm{d} t}, \quad \text { where } \quad \frac{\partial(\operatorname{det} \mathbb{F})}{\partial \mathbb{F}}=(\operatorname{det} \mathbb{F}) \mathbb{F}^{-t}
$$

Here, the symbol : denotes the inner product between tensors, i.e., $\mathbb{A}: \mathbb{B}=\operatorname{tr}\left(\mathbb{A}^{t} \mathbb{B}\right)$, where $\operatorname{tr}$ is the trace operator. Finally, substitution of the GCL (7) into the foregoing equation yields the partial differential equation satisfied by the Jacobian of the deformation gradient

$$
\frac{\mathrm{d} J}{\mathrm{~d} t}-J \operatorname{tr}(\mathbb{L})=0
$$

Because $\operatorname{tr}(\mathbb{L})=\nabla_{x} \cdot \boldsymbol{v}$, the time rate of change of the Jacobian can be rewritten as follows

$$
\frac{\mathrm{d} J}{\mathrm{~d} t}-J \nabla_{x} \cdot \boldsymbol{v}=0
$$

The second consequence of (7) is related to the computation of the time rate of change of the left Cauchy-Green tensor, $\mathbb{B}=\mathbb{F F}^{t}$, which reads

$$
\frac{\mathrm{d} \mathbb{B}}{\mathrm{d} t}=\frac{\mathrm{d} \mathbb{F}}{\mathrm{d} t} \mathbb{F}^{t}+\mathbb{F} \frac{\mathrm{d} \mathbb{F}^{t}}{\mathrm{~d} t} .
$$

Substituting the expression of the time rate of change of $\mathbb{F}$ provided by the GCL into the foregoing equation leads to

$$
\frac{\mathrm{d} \mathbb{B}}{\mathrm{d} t}-\mathbb{L} \mathbb{B}-\mathbb{B L}^{t}=0 .
$$

The left-hand side of this equation is nothing but the Lie derivative of $\mathbb{B}$ otherwise named the Oldroyd rate of $\mathbb{B}[35]$. 


\subsection{Governing equations}

This section aims at briefly recalling the conservation laws and the constitutive law governing the time evolution of an isotropic material undergoing large deformations. The interested reader might consult [35] for further details regarding their derivation.

\subsubsection{Conservation laws}

Under the updated Lagrangian representation, the conservation laws of mass, momentum and total energy write

$$
\begin{aligned}
& \rho \frac{\mathrm{d} \tau}{\mathrm{d} t}-\nabla \cdot \boldsymbol{v}=0, \\
& \rho \frac{\mathrm{d} \boldsymbol{v}}{\mathrm{d} t}-\nabla \cdot \mathbb{T}=\mathbf{0}, \\
& \rho \frac{\mathrm{d} e}{\mathrm{~d} t}-\nabla \cdot(\mathbb{T} \boldsymbol{v})=0 .
\end{aligned}
$$

Here, the symbol $\frac{\mathrm{d}}{\mathrm{d} t}$ denotes the material derivative defined by (2), $\rho>0$ is the mass density and $\tau=\frac{1}{\rho}$ the specific volume. The specific total energy is $e=\varepsilon+\frac{1}{2} \boldsymbol{v}^{2}$ where $\varepsilon$ denotes the specific internal energy. The Cauchy stress tensor, $\mathbb{T}$, is symmetric, i.e., $\mathbb{T}=\mathbb{T}^{t}$, which ensures the conservation of angular momentum. Let us note that the nabla operator employed in the foregoing equations is expressed in terms of the Eulerian coordinate $\boldsymbol{x}$. This system of conservation laws written under updated Lagrangian representation is supplemented by the trajectory equation already introduced in (1), which is rewritten under the form

$$
\frac{\mathrm{d} \boldsymbol{x}}{\mathrm{d} t}=\boldsymbol{v}(\boldsymbol{x}(t), t), \quad \boldsymbol{x}(0)=\boldsymbol{X}
$$

It is worth pointing out that (10a) is obtained by combining the Lagrangian mass conservation equation, $\frac{\mathrm{d}(\rho J)}{\mathrm{d} t}=0$, and the GCL (8). To close the system of conservation laws, it remains to provide a constitutive law for expressing the Cauchy stress tensor in terms of the deformation and a thermodynamic variable. This will be achieved in the next paragraph introducing the free energy $\Psi$. This thermodynamic potential is related to the specific energy, the absolute temperature $\theta>0$, and the specific entropy $\eta$ by means of the classical thermodynamic relation

$$
\Psi=\varepsilon-\theta \eta
$$

\subsubsection{Constitutive law for isotropic materials}

The constitutive law is derived invoking the frame indifference principle and the compatibility with thermodynamics. This means that the constitutive equations should be invariant under changes of frame and satisfy the second law of thermodynamics [35]. Here, the material under consideration is characterized by the free energy expressed in terms of the left Cauchy-Green tensor and the absolute temperature

$$
\Psi \equiv \Psi(\mathbb{B}, \theta)
$$


Moreover, since this material is isotropic, its constitutive law is invariant under the group of all rotations acting in the spatial configuration. Thus, the theorem of representation of isotropic scalar function [35] leads to the following expression of the free energy

$$
\Psi \equiv \Psi\left(I_{1}(\mathbb{B}), I_{2}(\mathbb{B}), I_{3}(\mathbb{B}), \theta\right)
$$

Here, $I_{i}(\mathbb{B})$ for $i=1,2,3$ are the principal invariants of the left Cauchy-Green tensor which write

$$
\left.I_{1}(\mathbb{B})=\operatorname{tr}(\mathbb{B}), I_{2}(\mathbb{B})\right)=\frac{1}{2}\left[\operatorname{tr}^{2}(\mathbb{B})-\operatorname{tr}\left(\mathbb{B}^{2}\right)\right], I_{3}(\mathbb{B})=\operatorname{det}(\mathbb{B}) .
$$

Finally, the constitutive law provides the expressions of the Cauchy stress tensor and the specific entropy in terms of the free energy

$$
\mathbb{T}(\mathbb{B}, \theta)=2 \rho\left(\frac{\partial \Psi}{\partial \mathbb{B}}\right)_{\theta} \mathbb{B}, \quad \text { and } \quad \eta(\mathbb{B}, \theta)=-\left(\frac{\partial \Psi}{\partial \theta}\right)_{\mathbb{B}},
$$

where $\frac{\partial \Psi}{\partial \mathbb{B}}$ is the tensor whose ij component is $\frac{\partial \Psi}{\partial \mathbb{B}_{i j}}$. Thanks to (12), we observe that the specific internal energy $\varepsilon$ is also a function of the left Cauchy Green tensor and the temperature, i.e., $\varepsilon(\mathbb{B}, \theta)=\Psi(\mathbb{B}, \theta)+\theta \eta(\mathbb{B}, \theta)$.

The foregoing generic expression of the Cauchy stress tensor might be further investigated exploiting the isotropy of the material. Indeed, differentiating (13) with respect to $\mathbb{B}$ and applying the chain rule yields

$$
\left(\frac{\partial \Psi}{\partial \mathbb{B}}\right)_{\theta}=\left(\frac{\partial \Psi}{\partial I_{1}}\right)_{\theta} \mathbb{I}_{\mathrm{d}}+\left(\frac{\partial \Psi}{\partial I_{2}}\right)_{\theta}\left(I_{1} \mathbb{I}_{\mathrm{d}}-\mathbb{B}\right)+\left(\frac{\partial \Psi}{\partial I_{3}}\right)_{\theta} I_{3} \mathbb{B}^{-1}
$$

where the derivative of the principal invariants of $\mathbb{B}$ with respect to $\mathbb{B}$ are recalled in Appendix A. Substituting the foregoing equation into the constitutive law results in

$$
\mathbb{T}=2 \rho\left\{I_{3}\left(\frac{\partial \Psi}{\partial I_{3}}\right)_{\theta} \mathbb{I}_{\mathrm{d}}+\left[\left(\frac{\partial \Psi}{\partial I_{1}}\right)_{\theta}+I_{1}\left(\frac{\partial \Psi}{\partial I_{2}}\right)_{\theta}\right] \mathbb{B}-\left(\frac{\partial \Psi}{\partial I_{2}}\right)_{\theta} \mathbb{B}^{2}\right\} .
$$

This is the general expression of the Cauchy stress tensor for an isotropic hyperelastic material. It is quadratic with respect to the left Cauchy-Green tensor. Let us point out that the Cauchy stress tensor and the left Cauchy-Green tensor commute, i.e. $\mathbb{T B}=\mathbb{B} \mathbb{T}$. This important property is the consequence of the material isotropy.

It remains to check the consistency of this constitutive law with the second law of thermodynamics. First, differentiation of the definition of the free energy (12) yields

$$
\begin{aligned}
\theta \mathrm{d} \eta & =\mathrm{d} \varepsilon-\mathrm{d} \Psi-\eta \mathrm{d} \theta \\
& =\mathrm{d} \varepsilon-\frac{\partial \Psi}{\partial \mathbb{B}}: \mathrm{d} \mathbb{B}-\frac{\partial \Psi}{\partial \theta} \mathrm{d} \theta-\eta \mathrm{d} \theta, \quad \text { since } \quad \Psi=\Psi(\mathbb{B}, \theta) .
\end{aligned}
$$

Substituting the constitutive law (14) in the above equation and recalling that $\varepsilon=e-\frac{1}{2} \boldsymbol{v}^{2}$, we arrive at the fundamental Gibbs relation

$$
\theta \mathrm{d} \eta=-\frac{1}{2 \rho} \mathbb{T B}^{-1}: \mathrm{d} \mathbb{B}-\boldsymbol{v} \cdot \mathrm{d} \boldsymbol{v}+\mathrm{d} e .
$$


The Gibbs relation enables us to compute the time rate of change of entropy as follows

$$
\rho \theta \frac{\mathrm{d} \eta}{\mathrm{d} t}=-\frac{1}{2} \mathbb{T B}^{-1}: \frac{\mathrm{d} \mathbb{B}}{\mathrm{d} t}-\rho \boldsymbol{v} \cdot \frac{\mathrm{d} \boldsymbol{v}}{\mathrm{d} t}+\rho \frac{\mathrm{d} e}{\mathrm{~d} t} .
$$

On the one hand, substituting the GCL (9) into the first term of the right-hand side of (17) leads to

$$
\begin{aligned}
\frac{1}{2} \mathbb{T B}^{-1}: \frac{\mathrm{d} \mathbb{B}}{\mathrm{d} t} & =\frac{1}{2} \mathbb{T B}^{-1}:\left(\mathbb{L} \mathbb{B}-\mathbb{B L}^{t}\right) \\
& =\mathbb{T}: \mathbb{L}, \quad \text { since } \quad \mathbb{T} \text { and } \mathbb{B} \text { commute. }
\end{aligned}
$$

On the other hand, substituting the conservation laws (10b) and (10c) into the remaining terms of the right-hand side of (17) yields

$$
\begin{aligned}
-\rho \boldsymbol{v} \cdot \frac{\mathrm{d} \boldsymbol{v}}{\mathrm{d} t}+\rho \frac{\mathrm{d} e}{\mathrm{~d} t} & =-\boldsymbol{v} \cdot \nabla \cdot(\mathbb{T})+\nabla \cdot(\mathbb{T} \boldsymbol{v}), \\
& =\mathbb{T}: \nabla \boldsymbol{v} .
\end{aligned}
$$

Here, we have employed the identity $\nabla \cdot\left(\mathbb{T}^{t} \boldsymbol{v}\right)=\boldsymbol{v} \cdot \nabla \cdot(\mathbb{T})+\mathbb{T}: \nabla \boldsymbol{v}$. Finally, gathering the foregoing results and observing that $\mathbb{L}=\nabla \boldsymbol{v}$ we arrive at

$$
\rho \theta \frac{\mathrm{d} \eta}{\mathrm{d} t}=0
$$

This shows that the system of conservation laws (10) is equipped with a supplementary conservation law which states that entropy is conserved along flow trajectories. Thus, constitutive law (14) for isotropic materials is consistent with the second law of thermodynamics. Let us point out that the algebraic manipulations which led to this result have been completed under the smoothness assumption of the flow variables. In the presence of discontinuities such as shock waves, the entropy conservation law turns into the entropy inequality

$$
\rho \theta \frac{\mathrm{d} \eta}{\mathrm{d} t} \geq 0
$$

\subsubsection{Volumetric shear strain decomposition}

We want to study materials that can sustain only limited shear strain but respond elastically to large change in volume. Following [50], we introduce the additive decomposition of the free energy into a volumetric part and a shear part. This also provides the additive decomposition of the Cauchy stress into a spherical part, which is nothing but the pressure, and a deviatoric part. To construct this additive decomposition, we start by introducing the multiplicative decomposition of the deformation gradient tensor, $\mathbb{F}$, into a volumetric and an isochoric parts. The volumetric part is equal to $J^{\frac{1}{3}} \mathbb{I}_{\mathrm{d}}$, whereas its isochoric part reads $\overline{\mathbb{F}}=J^{-\frac{1}{3}} \mathbb{F}$. This part of the deformation gradient is volume preserving since $\operatorname{det}(\overline{\mathbb{F}})=1$. This in turn implies that the isochoric part of the left Cauchy-Green tensor reads $\overline{\mathbb{B}}=J^{-\frac{2}{3}} \mathbb{B}$. Bearing this decomposition in mind, the expression of the free energy (13) turns into

$$
\Psi \equiv \Psi\left(J, I_{1}(\overline{\mathbb{B}}), I_{2}(\overline{\mathbb{B}}), \theta\right)
$$


The dependence of the free energy on $I_{3}$ is held by $J$ since $I_{3}(\overline{\mathbb{B}})=\operatorname{det}(\overline{\mathbb{B}})=1$. Now, we decompose this latter expression of the free energy into

$$
\Psi=\Psi_{v}(J, \theta)+\Psi_{s}\left(\bar{I}_{1}, \bar{I}_{2}, \theta\right)
$$

where $\Psi_{v}$ and $\Psi_{s}$ denote respectively the volumetric and the shear part of the free energy knowing that $\bar{I}_{1}=I_{1}(\overline{\mathbb{B}})$ and $\bar{I}_{2}=I_{2}(\overline{\mathbb{B}})$ are the principal invariants of the isochoric part of the left Cauchy-Green tensor $\overline{\mathbb{B}}$ (refer to Appendix A for the definition of the principal invariants of a tensor).

Finally, substituting the volumetric/shear decomposition of the free energy into the constitutive law (14) and after some algebra we arrive at

$$
\mathbb{T}=\rho J\left(\frac{\partial \Psi_{v}}{\partial J}\right)_{\theta} \mathbb{I}_{\mathrm{d}}+2 \rho\left[\left(\frac{\partial \Psi_{s}}{\partial \bar{I}_{1}}\right)_{\theta} \overline{\mathbb{B}}_{0}-\left(\frac{\partial \Psi_{s}}{\partial \bar{I}_{2}}\right)_{\theta}\left(\overline{\mathbb{B}}^{-1}\right)_{0}\right]
$$

Here, for a tensor, the superscript 0 denotes its deviatoric part. Thus, $\mathbb{T}_{0}$ is the deviatoric part of the Cauchy stress tensor defined by $\mathbb{T}_{0}=\mathbb{T}-\frac{1}{3} \operatorname{tr}(\mathbb{T}) \mathbb{I}_{d}$ and obviously $\operatorname{tr}\left(\mathbb{T}_{0}\right)=0$. Let us note that the foregoing expression of Cauchy stress tensor in terms of $\overline{\mathbb{B}}^{-1}$ has been obtained thanks to the Cayley-Hamilton theorem, refer to Appendix A, which allows to write $\overline{\mathbb{B}}^{-1}=\overline{\mathbb{B}}^{2}-\bar{I}_{1} \overline{\mathbb{B}}+\bar{I}_{2} \mathbb{I}_{\mathrm{d}}$. Observing (22), we arrive at the conclusion that the Cauchy stress decomposes into a spherical and a deviatoric part which are respectively defined by

$$
\begin{array}{rr}
p=-\rho J\left(\frac{\partial \Psi_{v}}{\partial J}\right)_{\theta} & \text { spherical part } \\
\mathbb{T}_{0}=2 \rho\left(\frac{\partial \Psi_{S}}{\partial \bar{I}_{1}}\right)_{\theta} \overline{\mathbb{B}}_{0}-2 \rho\left(\frac{\partial \Psi_{S}}{\partial \bar{I}_{2}}\right)_{\theta}\left(\overline{\mathbb{B}}^{-1}\right)_{0}, & \text { deviatoric part. }
\end{array}
$$

Here, $p=p(J, \theta)$ is nothing but the pressure and we point out that $\mathbb{T}_{0}=\mathbb{T}_{0}\left(\bar{I}_{1}, \bar{I}_{2}, \theta\right)$.

Remark 1 (Hyper-elasticity versus hypo-elasticity.) Hyperelasticity relies on the definition of a free energy which allows to express the deviatoric part of the Cauchy stress in terms of the deviatoric part of the left Cauchy-Green tensor. This framework provides a constitutive law fulfilling

- the material frame indifference principle;

- the thermodynamic consistency with the second law.

On the other hand, for hypo-elasticity, refer for instance to [47], the constitutive law is written under incremental form. Namely, the time rate of change of the deviatoric stress is expressed in terms of the deviatoric part of the strain rate tensor. The enforcement of the principle of material frame indifference relies on the use of a somewhat arbitrary objective stress rate such as the Jaumann rate, refer to [35]. Moreover, the use of objective stress rate makes appearing non conservative terms which render the mathematical analysis of discontinuous solutions quite delicate. This framework does not allow the fulfilment of thermodynamic consistency. Indeed, for smooth elastic flows the entropy is not conserved.

According to the constitutive law (14) the volumetric/shear decomposition of the free energy 
also induces a similar additive decomposition of the specific entropy $\eta=\eta_{v}+\eta_{s}$ where

$$
\begin{aligned}
\eta_{v}(J, \theta) & =-\left(\frac{\partial \Psi_{v}}{\partial \theta}\right)_{J}, \quad \text { volumetric part } \\
\eta_{s}\left(\bar{I}_{1}, \bar{I}_{2}, \theta\right) & =-\left(\frac{\partial \Psi_{s}}{\partial \theta}\right)_{\bar{I}_{1}, \bar{I}_{2}}, \quad \text { shearing part. }
\end{aligned}
$$

Gathering the foregoing results and recalling that, $\varepsilon=\Psi+\theta \eta$, leads to

$$
\varepsilon=\Psi_{v}+\Psi_{s}+\theta\left(\eta_{v}+\eta_{s}\right)=\left(\Psi_{v}+\theta \eta_{v}\right)+\left(\Psi_{s}+\theta \eta_{s}\right) .
$$

Thus, it is natural to introduce the volumetric and the shearing parts of the specific internal energy as follows

$$
\begin{aligned}
\varepsilon_{v}(J, \theta) & =\Psi_{v}(J, \theta)+\theta \eta_{v}(J, \theta), \\
\varepsilon_{s}\left(\bar{I}_{1}, \bar{I}_{2}, \theta\right) & =\Psi_{s}\left(\bar{I}_{1}, \bar{I}_{2}, \theta\right)+\theta \eta_{s}\left(\bar{I}_{1}, \bar{I}_{2}, \theta\right) .
\end{aligned}
$$

Remark 2 (About other thermodynamic potentials.) The thermoelastic response of the material could have been defined choosing internal energy, $\varepsilon \equiv \varepsilon(\mathbb{B}, \eta)$, as a thermodynamic potential to further derive the constitutive law, refer for instance to [30,39]. However, as noticed in [50], such a choice is inappropriate because it would imply that the absolute temperature $\theta$ (which is an intensive thermodynamic quantity) is a sum of volumetric/shear contributions. Moreover, the choice of the absolute temperature as an independent variable is more convenient since the notion of stress depending on temperature is more familiar, mostly because the temperature can easily be measured with classical devices.

\subsubsection{Examples of constitutive laws}

Let us point out that the volumetric/shear decomposition allows us to define separately the pressure by introducing an hydrodynamic equation of state characterized by the volumetric free energy $\Psi_{v}=\Psi_{v}(J, \theta)$. The pressure and the internal energy are expressed by means of classical thermodynamic relations

$$
p(\tau, \theta)=-\rho^{0}\left(\frac{\partial \Psi_{v}}{\partial J}\right)_{\theta}, \quad \varepsilon_{v}(J, \theta)=\Psi_{v}(J, \theta)-\theta\left(\frac{\partial \Psi_{v}}{\partial \theta}\right)_{J},
$$

where $\rho^{0}>0$ denotes the initial mass density of the solid. In what follows, for numerical applications, we shall make use of the volumetric free energy

$$
\Psi_{v}=\frac{\mu}{4 \rho^{0}}\left((J-1)^{2}+(\log J)^{2}\right),
$$

which leads to the pressure $p=-\frac{\mu}{2}\left(J-1+\frac{\log J}{J}\right)$ and the volumetric internal energy $\varepsilon_{v}=\Psi_{v}$. Apart from this equation of state, we shall also utilize the stiffened gas equation of state, which writes under the incomplete form

$$
\varepsilon_{v}=\frac{p+\gamma p_{\infty}}{(\gamma-1) \rho}
$$

where $\gamma$ and $p_{\infty}$ are material-dependent parameters. More generally, one can utilize one's favorite equation of state regardless of the shearing free energy choice. However, one shall 
always choose at least a convex equation of state to ensure the hyperbolicity of the hydrodynamic part of the system of conservation laws.

Regarding the shear part of the free energy we use the family of rank-one convex stored energies proposed in [29]

$$
\Psi_{s}\left(\bar{I}_{1}, \bar{I}_{2}\right)=\frac{\mu}{4 \rho^{0}}\left[-2 a\left(\bar{I}_{1}-3\right)+\frac{(1+a)}{3}\left(\bar{I}_{2}^{2}-9\right)\right]
$$

where $a$ is an adjustable parameter. For $a \in\left[-1, \frac{1}{2}\right]$, it is shown in [29] that the resulting system of conservation laws is hyperbolic. For the numerical applications, we shall consider the particular case $a=-1$ which corresponds to neo-Hookean materials. In this case, the shear part of free energy reads $\Psi_{s}=\frac{\mu}{2 \rho^{0}}\left(\bar{I}_{1}-3\right)$ and thus the deviatoric part of the Cauchy stress tensor is given by

$$
\mathbb{T}_{0}=\frac{\mu}{J} \overline{\mathbb{B}}_{0}
$$

where $\overline{\mathbb{B}}_{0}=\overline{\mathbb{B}}-\frac{1}{3} \operatorname{tr}(\overline{\mathbb{B}}) \mathbb{I}_{\mathrm{d}}$.

Finally, material mechanical properties are often described in terms of Young modulus E, Poisson ration $\nu$ and shear modulus $\mu$, which also corresponds to the second Lamé coefficient. These parameters are linked as follows:

$$
\mu=\frac{E}{2(1+\nu)} .
$$

In this paper, the numerical simulations will be carried out mainly with the neo-Hookean hyperelastic constitutive law, however we might also employ the non linear constitutive law (29) in the case $a=0$ for comparison purposes.

\subsection{Updated Lagrangian hyperelasticity for isotropic materials}

We summarize the set of partial differential equations governing the time evolution of the isotropic hyperelastic material under consideration. The conservation laws of mass, momentum and total energy read

$$
\begin{aligned}
& \rho \frac{\mathrm{d} \tau}{\mathrm{d} t}-\nabla \cdot \boldsymbol{v}=0, \\
& \rho \frac{\mathrm{d} \boldsymbol{v}}{\mathrm{d} t}-\nabla \cdot \mathbb{T}=\mathbf{0}, \\
& \rho \frac{\mathrm{d} e}{\mathrm{~d} t}-\nabla \cdot(\mathbb{T} \boldsymbol{v})=0 .
\end{aligned}
$$

This system is supplemented by the trajectory equation

$$
\frac{\mathrm{d} \boldsymbol{x}}{\mathrm{d} t}=\boldsymbol{v}(\boldsymbol{x}(t), t), \boldsymbol{x}(0)=\boldsymbol{X}
$$

The Cauchy stress tensor is symmetric, i.e., $\mathbb{T}=\mathbb{T}^{t}$. It is obtained deriving the free energy with respect to the left Cauchy-Green tensor $\mathbb{B}$. Assuming a volumetric/shear decomposition of the free energy, $\Psi=\Psi_{v}+\Psi_{s}$, the Cauchy stress tensor reads

$$
\mathbb{T}=\rho J\left(\frac{\partial \Psi_{v}}{\partial J}\right)_{\theta} \mathbb{I}_{\mathrm{d}}+2 \rho\left[\left(\frac{\partial \Psi_{s}}{\partial \bar{I}_{1}}\right)_{\theta} \overline{\mathbb{B}}_{0}-\left(\frac{\partial \Psi_{s}}{\partial \bar{I}_{2}}\right)_{\theta}\left(\overline{\mathbb{B}}^{-1}\right)_{0}\right] .
$$


Here, $\overline{\mathbb{B}}=J^{-\frac{2}{3}} \mathbb{B}$ denotes the isochoric part of the left Cauchy-Green tensor and $\bar{I}_{1}, \bar{I}_{2}$ are respectively its first and second invariants. We note also that $\Psi_{v}=\Psi_{v}(J, \theta)$ and $\Psi_{s}=$ $\Psi_{s}\left(\bar{I}_{1}, \bar{I}_{2}, \theta\right)$. By construction, the foregoing constitutive law satisfies the material frame indifference principle and is thermodynamically consistent which allows to write the Gibbs identity

$$
\theta \mathrm{d} \eta=-\frac{1}{2 \rho} \mathbb{T B}^{-1}: \mathrm{d} \mathbb{B}-\boldsymbol{v} \cdot \mathrm{d} \boldsymbol{v}+\mathrm{d} e .
$$

This system of physical conservation laws is completed by the geometrical conservation law expressing the time rate of change of the left Cauchy-Green tensor

$$
\frac{\mathrm{d} \mathbb{B}}{\mathrm{d} t}-\mathbb{L} \mathbb{B}-\mathbb{B L}^{t}=0
$$

where $\mathbb{L}=\nabla \boldsymbol{v}$ is the Eulerian velocity gradient tensor.

It is remarkable to note that updated Lagrangian isotropic hyperelasticity requires only the knowledge of the left Cauchy-Green tensor.

Remark 3 (Physical admissibility.) The physical admissibility property is defined by a set of so-called admissible states such that the material vector determines a valid state according to the conservation and constitutive laws. If the vector of variables is $\mathbf{Q}=(\tau, \boldsymbol{v}, e, \mathbb{B})$ supplemented with its relationships with derived variables, $\varepsilon, \mathbb{L}$, etc. in the hyperelastic model considered in this work the physically admissible set $\mathcal{A}$ is

$$
\mathcal{A}=\left\{\text { Q s.t. } \tau>0 \text { and } \varepsilon=e-\frac{1}{2} \boldsymbol{v}^{2}>0 \text { and } \theta>0 \text { and } \rho \theta \frac{\mathrm{d} \eta}{\mathrm{d} t} \geq 0\right\} .
$$

\section{Finite volume discretization}

Let $\omega(t)$ be the computational domain at time $t>0$. It is a subset of the three-dimensional space whose boundary surface $\partial \omega(t)$ is characterized by the outward pointing unit normal vector $\boldsymbol{n}$.

\subsection{Mesh and notation}

The computational domain $\omega(t)$ is discretized at time $t \geq 0$ by a set of non-overlapping simplicial cells. $N_{E}$ represents the total number of elements/cells in the domain and a cell is referred to with label $c$, that is $\omega_{c}(t)$. We also refer to a vertex/point with index $p$. Moreover the set of points of a cell is denoted by $\mathcal{P}(c)$ and the set of cells sharing a given point $p$ is $\mathcal{C}(p)$. Next the set of the faces of a cell is $\mathcal{F}(c)$ and the set of faces sharing a node $p$ is $\mathcal{F}(p)$. Likewise the set of edges of a cell is $\mathcal{E}(c)$, and the set of those impinging at a common point is denoted by $\mathcal{E}(p)$.

For any discrete time $t^{n}, n \in \mathbb{N}$, the union of all elements $\omega_{c}^{n}:=\omega_{c}\left(t^{n}\right)$ paving $\omega\left(t^{n}\right)$ is called the current mesh configuration $\mathcal{T}_{\omega}^{n}$ of the domain

$$
\mathcal{T}_{\omega}^{n}=\bigcup_{c=1}^{N_{E}} \omega_{c}^{n}
$$

Each control volume defined in the physical space $\boldsymbol{x}=(x, y, z)$ can be mapped onto a reference element $\omega_{e}$ in the reference coordinate system $\boldsymbol{\xi}=(\xi, \eta, \zeta)$ in $3 \mathrm{D}$, see figure 2 . 
In 2D the third components of $\boldsymbol{x}$ and $\boldsymbol{\xi}$ are maintained constant. We consider that any polyhedral cell can be split into tetrahedra, as such the method developed in this work is readily applicable on meshes made of polyhedra.

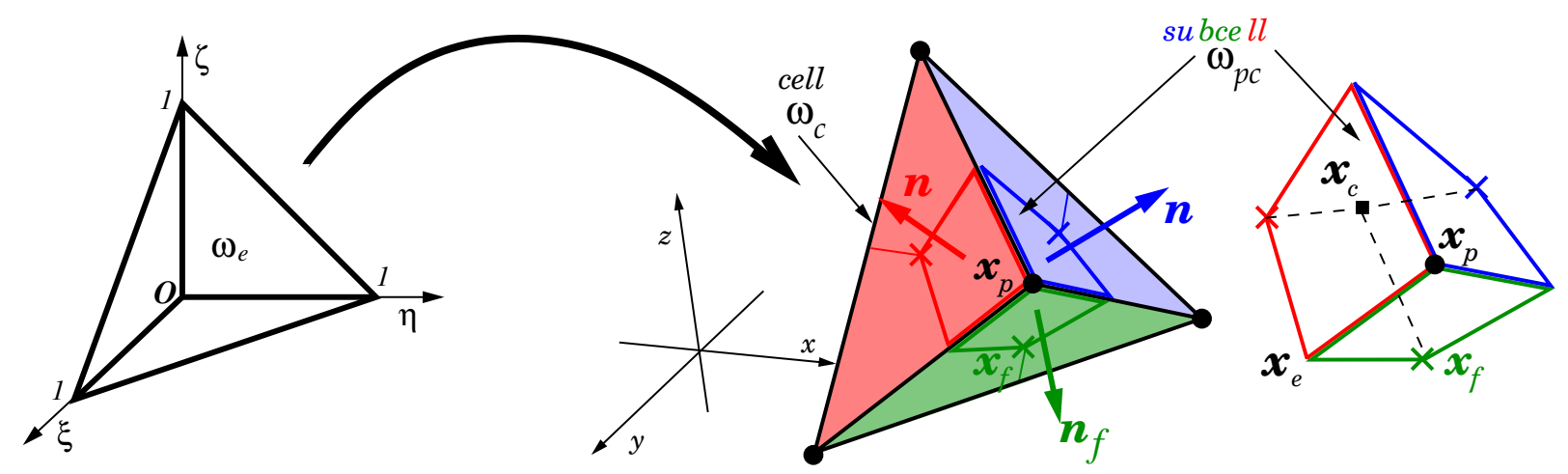

Fig. 2. Left: Reference simplicial element $\omega_{e}$ in coordinates $\boldsymbol{\xi}=(\xi, \eta, \zeta)-$ Right: cell $\omega_{c}$, subcell $\omega_{p c}$ and geometrical face/cell/point centers and outward pointing face normal $\boldsymbol{n}_{f}$ to each face $f$.

\subsubsection{Geometrical entities}

The volume of the cell $\omega_{c}(t)$ is denoted by $\left|\omega_{c}(t)\right|$. The cell center, $\boldsymbol{x}_{c}$, corresponds to its centroid, that is

$$
\boldsymbol{x}_{c}=\frac{1}{\left|\omega_{c}(t)\right|} \int_{\omega_{c}(t)} \boldsymbol{x} \mathrm{d} v
$$

The center of a face $f$ is the iso-barycenter of the points defining the face: $\boldsymbol{x}_{f}=\frac{1}{|\mathcal{P}(f)|} \sum_{p \in \mathcal{P}(f)} \boldsymbol{x}_{p}$, where $|\mathcal{S}|$ denotes the cardinal of any set $\mathcal{S}$.

Given a cell $c$ and a point $p$ we define a unique object called subcell, referred to with double index $p c$ which is the unique geometrical object linking the cell center $\boldsymbol{x}_{c}$, the point $\boldsymbol{x}_{p}$ and the face centers $\boldsymbol{x}_{f}$ for all face $f \in \mathcal{F}(p c)$ with $\mathcal{F}(p c) \equiv \mathcal{F}(c) \cap \mathcal{F}(p)$. In 3D the subcell is a hexaedron with possibly non-planar faces, in $2 \mathrm{D}$ it is a quadrangle, refer to figure 2 . A subcell is further denoted by $\omega_{p c}$, its volume is referred to as $\left|\omega_{p c}\right|$, see figure 2 . Each face $f \in \mathcal{F}(p c)$ of a subcell $\omega_{p} c$ is assigned a unique outward pointing unit normal $\boldsymbol{n}_{f}$ and surface $A_{f}>0$. As such any cell $\omega_{c}$ is a collection of subcells: $\omega_{c}=\bigcup_{p \in \mathcal{P}(c)} \omega_{p c}$, each being considered as a Lagrangian object.

In a Lagrangian framework the mass of a subcell and cell, $m_{p c}, m_{c}$ respectively, are constant in time and equal to

$$
m_{p c}=\int_{\Omega_{p c}} \rho^{0}(\boldsymbol{X}) \mathrm{d} V, \quad m_{c}=\int_{\Omega_{c}} \rho^{0}(\boldsymbol{X}) \mathrm{d} V=\sum_{p \in \mathcal{P}(c)} m_{p c}
$$

where $\Omega_{p c}$ and $\Omega_{c}$ denote the subcell and the cell in the initial configuration, $\rho^{0}(\boldsymbol{X})>0$ is the initial density distribution, and $\mathrm{d} V$ refers to the integral measure over volume.

The cell volume might be expressed in terms of its vertices location, that is

$$
\left|\omega_{c}(t)\right|=\left|\omega_{c}\left(\boldsymbol{x}_{1}(t), \cdots, \boldsymbol{x}_{|\mathcal{P}(c)|}(t)\right)\right| .
$$

Thus, applying the chain rule of composed derivative, the time rate of change of the cell 
volume writes

$$
\frac{\mathrm{d}\left|\omega_{c}(t)\right|}{\mathrm{d} t}=\sum_{p \in \mathcal{P}(c)} \frac{\partial\left|\omega_{c}\right|}{\partial \boldsymbol{x}_{p}} \cdot \frac{\mathrm{d} \boldsymbol{x}_{p}}{\mathrm{~d} t} .
$$

This equation allows us to distinguish the fundamental geometrical object

$$
a_{p c} \boldsymbol{n}_{p c}=\frac{\partial\left|\omega_{c}\right|}{\partial \boldsymbol{x}_{p}}
$$

known as the corner normal vector. It has been introduced by several authors in the context of Lagrangian hydrodynamics, refer for instance to [2]. Here, $a_{p c}$ represents a $(d-1)$-measure (length in 2D, area in 3D) and $\boldsymbol{n}_{p c}$ is a unit outward pointing vector. Algebraic manipulations of (37) may convince the reader that the corner vector is the sum of the face outward pointing normal vectors for all face $f \in \mathcal{F}(p c)$ of the current cell $c$ impinging on node $p$. The cell $\omega_{c}$ being a closed contour, the set of its corner normal vectors satisfies the fundamental geometrical identity

$$
\sum_{p \in \mathcal{P}(c)} a_{p c} \boldsymbol{n}_{p c}=\mathbf{0}
$$

Let us point out that the corner normal vector relative to the initial Lagrangian cell, $\Omega_{c}$, is similarly defined by

$$
A_{p c} \boldsymbol{N}_{p c}=\frac{\partial\left|\Omega_{c}\right|}{\partial \boldsymbol{X}_{p}}
$$

\subsubsection{Conservative and constitutive discrete variables}

The time dependent conserved or constitutive variables are the cell-centered approximate mass-averaged values gathered into vector $\mathbf{Q}_{c}(t)=\left(\tau_{c}(t), \boldsymbol{v}_{c}(t), e_{c}(t), \mathbb{B}_{c}(t)\right)$. For a vector or a tensor this should be understood as component-wise. We also use in this work a point-wise velocity field $\boldsymbol{v}_{p}$ which represents the velocity of point $p$, i.e., $\boldsymbol{v}_{p}=\frac{\mathrm{d} \boldsymbol{x}_{p}}{\mathrm{~d} t}$.

From now on we implicitly assume the dependence on time and to lighten the notation we omit it.

\subsection{Discrete velocity gradient operators}

This paragraph aims at presenting a FV approximation of the velocity gradient for the updated Lagrangian representation. This operator is of great importance for deriving moving grid discretization of Lagrangian hydrodynamics, see for instance $[47,42]$. The resulting discrete velocity gradient operator shall be useful for the space discretization of the Geometric Conservation Law which governs the time rate of change of the deformation gradient. Application of the divergence theorem leads to write the updated Lagrangian cell-centered velocity gradient operator

$$
\mathbb{L}_{c}(\boldsymbol{v})=\frac{1}{\left|\omega_{c}\right|} \int_{\partial \Omega_{c}} \boldsymbol{v} \otimes \boldsymbol{n} \mathrm{d} s .
$$

Let us note that the velocity field is defined by its values at the cell nodes denoted by $\boldsymbol{v}_{p}=\boldsymbol{v}\left(\boldsymbol{x}_{p}\right)$. Now, approximating the foregoing integrals thanks to the corner normal vectors (37) and the pointwise velocity field we arrive at the approximation of the discrete Lagrangian 
and Eulerian velocity gradient operators

$$
\mathbb{L}_{c}(\boldsymbol{v})=\frac{1}{\left|\omega_{c}\right|} \sum_{p \in \mathcal{P}(c)} a_{p c} \boldsymbol{v}_{p} \otimes \boldsymbol{n}_{p} .
$$

It is worth pointing out that this discrete gradient operator is exact for affine velocity fields. This property relies on the geometrical identities (38) and

$$
\sum_{p \in \mathcal{P}(c)} a_{p c} \boldsymbol{x}_{p} \otimes \boldsymbol{n}_{p c}=\left|\omega_{c}\right| \mathbb{I}_{\mathrm{d}}
$$

The interested reader might refer for instance to [22] for finding the demonstration of this result.

We end up presenting an important by-product of the discrete Eulerian velocity gradient operator. Taking the trace of (40) leads to

$$
\operatorname{tr}\left[\mathbb{L}_{c}(\boldsymbol{v})\right]=\frac{1}{\left|\omega_{c}\right|} \sum_{p \in \mathcal{P}(c)} a_{p c} \boldsymbol{v}_{p} \cdot \boldsymbol{n}_{p c}
$$

This is nothing but the discrete cell-centered divergence operator. Finally, combining the definition of the Eulerian corner normal vector (37) and the discrete divergence operator leads to rewrite the time rate of change of the cell volume as follows

$$
\frac{1}{\left|\omega_{c}\right|} \frac{\mathrm{d}\left|\omega_{c}\right|}{\mathrm{d} t}=\operatorname{tr}\left[\mathbb{L}_{c}(\boldsymbol{v})\right]
$$

\subsection{Semi-discretization in space}

\subsubsection{Conservation laws - GCL, momentum and total energy}

The geometrical conservation law (GCL) is a fundamental consistency property in Lagrangian framework. Indeed it states that the discrete motion of all the points $p$ of a given cell $\omega_{c}$ with the trajectory equations

$$
\frac{\mathrm{d} \boldsymbol{x}_{p}}{\mathrm{~d} t}=\boldsymbol{v}_{p}
$$

is consistent with the volume conservation law (10a). Since $m_{c} \tau_{c}=\left|\omega_{c}\right|$ and taking into account (41), we readily infer the discrete version of the volume conservation law which is compatible with the GCL

$$
m_{c} \frac{\mathrm{d} \tau_{c}}{\mathrm{~d} t}-\sum_{p \in \mathcal{P}(c)} a_{p c} \boldsymbol{n}_{p c} \cdot \boldsymbol{v}_{p}=0 .
$$

The space discretization of the momentum and the total energy equations is performed introducing the subcell force $\boldsymbol{f}_{p c}$, which is the traction force exerted on the outer boundary of the subcell $\omega_{p c}$. More precisely, starting from (10b) further integrated over cell $\omega_{c}$ and invoking Green theorem we formally can get

$$
m_{c} \frac{\mathrm{d} \boldsymbol{v}_{c}}{\mathrm{~d} t}-\int_{\partial \omega_{c}} \mathbb{T} \boldsymbol{n} \mathrm{d} s=\mathbf{0}
$$


Then, the previous integral over the cell boundary is split over the subcell boundaries and one gets

$$
m_{c} \frac{\mathrm{d} \boldsymbol{v}_{c}}{\mathrm{~d} t}-\sum_{p \in \mathcal{P}(c)} \int_{\partial \omega_{p c} \cap \partial \omega_{c}} \mathbb{T} \boldsymbol{n} \mathrm{d} s=\mathbf{0},
$$

which yields our definition of the subcell force as

$$
\boldsymbol{f}_{p c}=\int_{\partial \omega_{p c} \cap \partial \omega_{c}} \mathbb{T} \boldsymbol{n} \mathrm{d} s
$$

Next, considering the energy equation (10c) and the same algebraic manipulation yields

$$
m_{c} \frac{\mathrm{d} e_{c}}{\mathrm{~d} t}-\sum_{p \in \mathcal{P}(c)} \int_{\partial \omega_{p c} \cap \partial \omega_{c}} \mathbb{T} \boldsymbol{v} \cdot \boldsymbol{n} \mathrm{d} s=0 .
$$

Assuming that the velocity along the faces impinging on point $\boldsymbol{x}_{p}$ is the constant point velocity $\boldsymbol{v}_{p}$, then the previous integral can be approximated as

$$
\int_{\partial \omega_{p c} \cap \partial \omega_{c}} \mathbb{T} \boldsymbol{v} \cdot \boldsymbol{n} \mathrm{d} s \simeq\left(\int_{\partial \omega_{p c} \cap \partial \omega_{c}} \mathbb{T} \boldsymbol{n} \mathrm{d} s\right) \cdot \boldsymbol{v}_{p}=\boldsymbol{f}_{p c} \cdot \boldsymbol{v}_{p}
$$

Therefore, using the subcell force definition, the semi-discrete version of the conservation laws read

$$
\begin{array}{r}
m_{c} \frac{\mathrm{d} \tau_{c}}{\mathrm{~d} t}-\sum_{p \in \mathcal{P}(c)} a_{p c} \boldsymbol{n}_{p c} \cdot \boldsymbol{v}_{p}=0, \\
m_{c} \frac{\mathrm{d} \boldsymbol{v}_{c}}{\mathrm{~d} t}-\sum_{p \in \mathcal{P}(c)} \boldsymbol{f}_{p c}=\mathbf{0}, \\
m_{c} \frac{\mathrm{d} e_{c}}{\mathrm{~d} t}-\sum_{p \in \mathcal{P}(c)} \boldsymbol{f}_{p c} \cdot \boldsymbol{v}_{p}=0 .
\end{array}
$$

This system of semi-discrete physical conservation laws is supplemented with the semidiscrete version of (9) expressing the time rate of change of the left Cauchy Green tensor required for the constitutive law

$$
\frac{\mathrm{d} \mathbb{B}_{c}}{\mathrm{~d} t}-\mathbb{L}_{c} \mathbb{B}_{c}-\mathbb{B}_{c} \mathbb{L}_{c}^{t}=0
$$

Here, $\mathbb{B}_{c}$ and $\mathbb{L}_{c}$ denote respectively the cell-centered values of the left Cauchy-Green and the Eulerian velocity gradient tensors. The discretization of this equation, which relies on geometrical considerations, shall be detailed in section 3.4.2. Let us point out that the remaining unknowns in the foregoing set of semi-discrete equations are the subcell force and the node velocity. We shall determine them invoking:

- The thermodynamic consistency of the semi-discrete scheme;

- The conservation of total energy and momentum.

The first requirement allows us to express the subcell force $\boldsymbol{f}_{p c}$ in terms of the node velocity $\boldsymbol{v}_{p}$. The second one provides a linear system at each node satisfied by the node velocity. The interested reader might refer to $[45,13]$ for a detailed presentation of this particular discretization. 


\subsubsection{The subcell force expression ensuring thermodynamic consistency}

The constitutive law leads to the definition of the following discrete Cauchy stress tensor: $\mathbb{T}_{c}=2 \rho_{c} \frac{\partial \Psi}{\partial \mathbb{B}}\left(\mathbb{B}_{c}, \theta_{c}\right) \mathbb{B}_{c}$. Starting from the Gibbs identity (16) let us compute the time evolution of the entropy within cell $\omega_{c}$

$$
m_{c} \theta_{c} \frac{\mathrm{d} \eta_{c}}{\mathrm{~d} t}=-\frac{1}{2}\left|\omega_{c}\right| \mathbb{T}_{c} \mathbb{B}_{c}^{-1}: \frac{\mathrm{d} \mathbb{B}_{c}}{\mathrm{~d} t}-m_{c} \boldsymbol{v}_{c} \cdot \frac{\mathrm{d} \boldsymbol{v}_{c}}{\mathrm{~d} t}+m_{c} \frac{\mathrm{d} e_{c}}{\mathrm{~d} t}
$$

Each term of the right hand side can be replaced by a more appropriate form for our analysis using (50), (51) and

$$
-\frac{1}{2} \mathbb{T}_{c} \mathbb{B}_{c}^{-1}: \frac{\mathrm{d} \mathbb{B}_{c}}{\mathrm{~d} t}=-\mathbb{T}_{c}: \mathbb{L}_{c}=-\mathbb{T}_{c}: \sum_{p \in \mathcal{P}(c)} a_{p c} \boldsymbol{v}_{p} \otimes \boldsymbol{n}_{p c}
$$

which after substitution yields

$$
\begin{aligned}
m_{c} \theta_{c} \frac{\mathrm{d} \eta_{c}}{\mathrm{~d} t} & =-\mathbb{T}_{c}: \sum_{p \in \mathcal{P}(c)} a_{p c} \boldsymbol{v}_{p} \otimes \boldsymbol{n}_{p c}+\sum_{p \in \mathcal{P}(c)} \boldsymbol{f}_{p c} \cdot\left(\boldsymbol{v}_{p}-\boldsymbol{v}_{c}\right) \\
& =-\sum_{p \in \mathcal{P}(c)}\left(\boldsymbol{v}_{p}-\boldsymbol{v}_{c}\right) \cdot \mathbb{T}_{c} \boldsymbol{n}_{p c}+\sum_{p \in \mathcal{P}(c)} \boldsymbol{f}_{p c} \cdot\left(\boldsymbol{v}_{p}-\boldsymbol{v}_{c}\right) \\
& =\sum_{p \in \mathcal{P}(c)}\left(-a_{p c} \mathbb{T}_{c} \boldsymbol{n}_{p c}+\boldsymbol{f}_{p c}\right) \cdot\left(\boldsymbol{v}_{p}-\boldsymbol{v}_{c}\right) .
\end{aligned}
$$

Therefore, in order to ensure a proper entropy dissipation, we propose to design the subcell force as

$$
\boldsymbol{f}_{p c}=a_{p c} \mathbb{T}_{c} \boldsymbol{n}_{p c}+\mathbb{M}_{p c}\left(\boldsymbol{v}_{p}-\boldsymbol{v}_{c}\right),
$$

where the subcell matrix $\mathbb{M}_{p c}$ is symmetric positive definite. We easily verify that

$$
m_{c} \theta_{c} \frac{\mathrm{d} \eta_{c}}{\mathrm{~d} t}=\sum_{p \in \mathcal{P}(c)} \mathbb{M}_{p c}\left(\boldsymbol{v}_{p}-\boldsymbol{v}_{c}\right) \cdot\left(\boldsymbol{v}_{p}-\boldsymbol{v}_{c}\right) \geq 0,
$$

which expresses the consistency with the second law of thermodynamics at the semi-discrete level. Now, it remains to determine the subcell matrix $\mathbb{M}_{p c}$, which genuinely characterizes the numerical scheme. Several possibilities have already been explored by different authors in $[44,18,52]$.

\subsubsection{The nodal solver ensuring the conservation of total energy and momentum}

Ignoring the boundary conditions, the conservation of total energy and momentum at the semi-discrete level is ensured provided that the sum of the subcell forces impinging at node $\boldsymbol{x}_{p}$ is equal to zero, that is

$$
\sum_{c \in \mathcal{C}(p)} \boldsymbol{f}_{p c}=\mathbf{0}
$$

The interested reader might refer for instance to $[46,45]$ for the justification of this result. Substituting the expression of the subcell force (54) in the foregoing condition leads to the linear system

$$
\mathbb{M}_{p} \boldsymbol{v}_{p}=\sum_{c \in \mathcal{C}(p)} \mathbb{M}_{p c} \boldsymbol{v}_{c}-\sum_{c \in \mathcal{C}(p)} a_{p c} \mathbb{T}_{c} \boldsymbol{n}_{p c}, \text { where } \quad \mathbb{M}_{p}=\sum_{c \in \mathcal{C}(p)} \mathbb{M}_{p c}
$$


Notice that $\mathbb{M}_{p}$ is symmetric positive definite and, thus, invertible. The subcell matrix in the present work writes

$$
\mathbb{M}_{p c}=\sum_{f \in \mathcal{F}(p c)} z_{f} A_{f} \boldsymbol{n}_{f} \otimes \boldsymbol{n}_{f}
$$

where we remind that $A_{f}$ is the surface of any of the three faces $f$ of the neighbor cells sharing point $p, \boldsymbol{n}_{f}$ is its outward unit normal and $z_{f} \equiv z_{c}=\rho_{c} \sqrt{a_{c}^{2}+\frac{4}{3} \frac{\mu_{c}}{\rho_{c}}}$ is an approximation of the swept mass flux, where $a_{c}$ is the local isentropic sound-speed and $\mu_{c}$ the cell shear modulus (31)). Once the node velocity is determined thanks to (57) then the trajectory equation can be invoked to update the node position.

\subsection{Space-Time discretization - ADER methodology}

\subsubsection{Physical conservation laws}

The time interval $[0, T]$ is discretized into time-steps such that $t \in\left[t^{n}, t^{n+1}\right]$,

$$
t=t^{n}+\alpha \Delta t, \quad \alpha \in[0,1]
$$

where $t^{n}$ and $\Delta t$ represent the current time and time-step respectively. For evaluating the magnitude of $\Delta t$ we use a classical CFL condition and a criterion to avoid a too large increase of cell volume in a single time-step [44], refer to section 3.7 where the time step monitoring is described. Knowing the state variable and the geometry at time $t^{n}$ we start by computing the nodal velocity $\boldsymbol{v}_{p}^{*}$ employing the nodal solver

$$
\mathbb{M}_{p} \boldsymbol{v}_{p}^{*}=\sum_{c \in \mathcal{C}(p)} \mathbb{M}_{p c} \boldsymbol{v}_{c}^{*}-a_{p c}^{n} \mathbb{T}_{c}^{*} \boldsymbol{n}_{p c}^{n}
$$

where the ${ }^{*}$ values on the right-hand side are obtained by high-accurate space-time reconstructions detailed below, moreover the discrete subcell matrix $\mathbb{M}_{p c}$ and nodal matrix $\mathbb{M}_{p}$ read

$$
\mathbb{M}_{p c}=\sum_{f \in \mathcal{F}(p c)} z_{f}^{*} A_{f}^{n} \boldsymbol{n}_{f}^{n} \otimes \boldsymbol{n}_{f}^{n}, \quad \mathbb{M}_{p}=\sum_{c \in \mathcal{C}(p)} \mathbb{M}_{p c}
$$

The knowledge of the node velocity allows us to compute the subcell force

$$
\boldsymbol{f}_{p c}^{*}=a_{p c}^{n} \mathbb{T}_{c}^{*} \boldsymbol{n}_{p c}^{n}+\mathbb{M}_{p c}\left(\boldsymbol{v}_{p}^{*}-\boldsymbol{v}_{c}^{*}\right)
$$

Integrating the trajectory equation yields the update node position

$$
\boldsymbol{x}_{p}^{n+1}=\boldsymbol{x}_{p}^{n}+\Delta t \boldsymbol{v}_{p}^{*},
$$

Then, it remains to perform the time explicit integration of (49), (50) and (51) to arrive at the updated values of the physical state variables

$$
\begin{aligned}
\tau_{c}^{n+1} & =\tau_{c}^{n}+\frac{\Delta t}{m_{c}} \sum_{p \in \mathcal{P}(c)} \widetilde{a_{p c} \boldsymbol{n}_{p c}} \cdot \boldsymbol{v}_{p}^{*} \\
\boldsymbol{v}_{c}^{n+1} & =\boldsymbol{v}_{c}^{n}+\frac{\Delta t}{m_{c}} \sum_{p \in \mathcal{P}(c)} \boldsymbol{f}_{p c}^{*} \\
e_{c}^{n+1} & =e_{c}^{n}+\frac{\Delta t}{m_{c}} \sum_{p \in \mathcal{P}(c)} \boldsymbol{f}_{p c}^{*} \cdot \boldsymbol{v}_{p}^{*} .
\end{aligned}
$$


According to (63), the coordinate position $\boldsymbol{x}_{p}$ is a linear function of time, thus the surface term, $\widetilde{a_{p c} \boldsymbol{n}_{p c}}$, in (64) exhibits a quadratic time dependency and, as such, must be exactly integrated in order to ensure the satisfaction of the GCL at the discrete level. Therefore, the time integral of $a_{p c} \boldsymbol{n}_{p c}$ over $\left[t^{n}, t^{n+1}\right]$ is computed at the aid of Simpson rule, which is

$$
\widetilde{a_{p c} \boldsymbol{n}_{p c}}=\frac{1}{\Delta t} \int_{t^{n}}^{t^{n+1}} a_{p c} \boldsymbol{n}_{p c} \mathrm{~d} t \approx \frac{1}{6} a_{p c}^{n} \boldsymbol{n}_{p c}^{n}+\frac{2}{3} a_{p c}^{n+\frac{1}{2}} \boldsymbol{n}_{p c}^{n+\frac{1}{2}}+\frac{1}{6} a_{p c}^{n+1} \boldsymbol{n}_{p c}^{n+1},
$$

that guarantees an exact integration up to third order. Notice that in 2D a midpoint rule is sufficient to ensure the formal accuracy of the method since the term $\widetilde{a_{p c} \boldsymbol{n}_{p c}}$ is linear in time.

The first-order time discretization simply considers $t^{*}=t^{n}$ and the cell-centered values of the state vector $\mathbf{Q}_{c}^{n}=\left(\tau_{c}, \boldsymbol{v}_{c}, e_{c}, \mathbb{B}_{c}\right)^{n}$. To obtain second order of accuracy in space, a piecewise linear reconstruction of the numerical solution $\mathbf{Q}_{c}$ must be carried out, while second-order time stepping demands that $t^{*}=t^{n+1 / 2}=\frac{1}{2}\left(t^{n}+t^{n+1}\right)$, which corresponds to the use of a midpoint rule to perform the time integration. Classically a predictor-corrector [16] or a Generalized-Riemann-Problem (GRP) scheme [44,14] are used for this matter. Contrarily, in this work, the second-order time discretization relies on the concept of the ADER (Arbitrary high order schemes using DERivatives) methodology following [13].

The ADER procedure aims at computing high order space-time polynomials $\mathbf{q}_{h}(\boldsymbol{x}, t)$ which are defined in terms of a nodal basis constituted of piecewise linear space-time basis functions $\theta(\boldsymbol{\xi}, \tau)$, that is

$$
\mathbf{q}_{h}=\sum_{l=1}^{\mathcal{L}} \theta_{l}(\boldsymbol{\xi}, \tau) \widehat{\mathbf{q}}_{l, c},
$$

where $\widehat{\mathbf{q}}_{l, c}$ are the $\mathcal{L}=2 \mathcal{M}$ degrees of freedom with $\mathcal{M}=d+1$. The space-time coordinate vector $(\boldsymbol{\xi}, \tau)=(\xi, \eta, \zeta, \tau)$ is defined in the spatial reference element given by the unit tetrahedron depicted in figure 2 and in the reference time $\tau \in[0 ; 1]$ which maps the time step $\left[t^{n} ; t^{n+1}\right]$. The space-time basis functions explicitly write in $3 \mathrm{D}$ for all $l \in[1 ; \mathcal{L}]$ as follows:

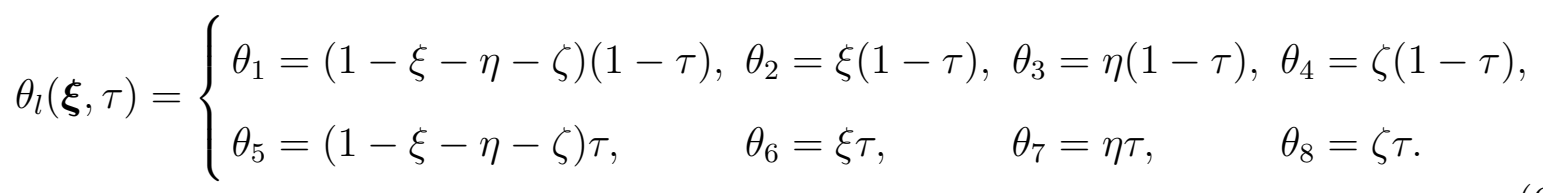

Spatial reconstruction. First, a piecewise linear spatial reconstruction is carried out [13], hence providing a second order polynomial approximation of the current numerical solution, i.e. $\mathbf{q}_{h}(\boldsymbol{\xi}, 0)$. This reconstruction is not limited, thus it can be efficiently obtained following the procedure detailed in [13]. The limiting will be done a posteriori via the MOOD paradigm. A so-called reconstruction stencil $\mathcal{S}_{c}=\bigcup_{j=1}^{n_{e}} \omega_{m(j)}^{n}$ is needed, where $1 \leq j \leq n_{e}$ is a local index that counts the elements belonging to the stencil, while $m(j)$ maps the local index to the global element numbers used in the mesh configuration (34). Notice that neither the stencil nor the element configuration is symmetric on unstructured meshes, thus, to avoid ill-conditioned reconstruction matrices, the stencil contains a total number of $n_{e}=d \mathcal{M}$ elements and is filled by the Voronoi neighbors of $\omega_{c}^{n}$, i.e., the neighbor elements sharing at least one vertex with element $\omega_{c}^{n}$ ). The reconstruction relies on integral conservation for 
each element $\omega_{j}^{n} \in \mathcal{S}_{c}$, that is

$$
\frac{1}{\left|\omega_{j}^{n}\right|} \int_{\omega_{j}^{n}} \sum_{l=1}^{\mathcal{L}} \theta_{l}(\boldsymbol{\xi}, 0) \widehat{\mathbf{q}}_{l, c} \mathrm{~d} v \simeq \mathbf{Q}_{j}^{n}, \quad \forall \omega_{j}^{n} \in \mathcal{S}_{c},
$$

with $\left|\omega_{j}^{n}\right|$ denoting the volume of element $\omega_{j}^{n}$ at time $t^{n}$. To enforce conservation of the reconstruction polynomial for the cell under consideration, the above integral conservation must hold exactly for cell $\omega_{c}^{n}$, hence requiring the additional linear constraint

$$
\frac{1}{\left|\omega_{c}^{n}\right|} \int_{\omega_{c}^{n}} \sum_{l=1}^{\mathcal{M}} \theta_{l}(\boldsymbol{\xi}, 0) \widehat{\mathbf{q}}_{l, c} \mathrm{~d} v=\mathbf{Q}_{c}^{n}
$$

The reconstruction system (70) is solved using a constrained least squares technique [11].

Time reconstruction. Once the spatial reconstruction polynomial $\mathbf{q}_{h}(\boldsymbol{\xi}, 0)$ is known, the ADER methodology performs a local time evolution of the governing equations (10)-(11)(32). To that aim, a weak formulation is derived by multiplying the PDE with a space-time test function $\theta_{k}(\boldsymbol{\xi}, \tau)$ that is

$$
\begin{aligned}
& \int_{t^{n}}^{t^{n+1}} \int_{\omega_{c}(t)} \theta_{k}(\boldsymbol{\xi}, \tau)\left(\frac{\mathrm{d} \mathbf{q}_{h}}{\mathrm{~d} t}-\frac{1}{\rho_{h}} \nabla \cdot \mathbf{f}\left(\mathbf{q}_{h}, \nabla \mathbf{q}_{h}\right)\right) \mathrm{d} v \mathrm{~d} t=0, \\
& \int_{t^{n}}^{t^{n+1}} \int_{\omega_{c}(t)} \theta_{k}(\boldsymbol{\xi}, \tau) \frac{\mathrm{d} \boldsymbol{x}}{\mathrm{d} t} \mathrm{~d} v \mathrm{~d} t=\int_{t^{n}}^{t^{n+1}} \int_{\omega_{c}(t)} \theta_{k}(\boldsymbol{\xi}, \tau) \boldsymbol{v} \mathrm{d} v \mathrm{~d} t
\end{aligned}
$$

with the state vector $\mathbf{q}_{h}=(\tau, \boldsymbol{v}, e, \mathbb{B})_{h}$ and the flux tensor $\mathbf{f}\left(\mathbf{q}_{h}, \nabla \mathbf{q}_{h}\right)=(\boldsymbol{v}, \mathbb{T}, \mathbb{T} \boldsymbol{v}, \mathbb{L} \mathbb{B}-$ $\left.\mathbb{B L}^{t}\right)_{h}$. The trajectory equation (71b) is coupled with the evolution of the governing PDE (71a), and the local element geometry is defined by $\boldsymbol{x}_{h}$ which is expressed in terms of the space-time basis functions as

$$
\boldsymbol{x}_{h}=\sum_{l=1}^{\mathcal{L}} \theta_{l}(\boldsymbol{\xi}, \tau) \widehat{\boldsymbol{x}}_{l, c} .
$$

The first $\mathcal{L} / 2$ degrees of freedom are known, since they correspond to the spatial reconstruction polynomial $\mathbf{q}_{h}(\boldsymbol{\xi}, 0)$ and to the vertex coordinates of the cell $\omega_{c}$ at time $t^{n}$, that is

$$
\widehat{\boldsymbol{x}}_{l, c}=\boldsymbol{x}_{p} \quad \text { such that } \quad p \in \mathcal{P}(c), \quad \forall l \in[1 ; \mathcal{L} / 2] .
$$

The above nonlinear system (71) can be compactly written in matrix-vector notation and then solved iteratively up to convergence for both the numerical solution $\mathbf{q}_{h}$ and the local geometry configuration $\boldsymbol{x}_{h}$, to obtain the remaining unknown $\mathcal{L} / 2$ expansion coefficients for $\tau>0$. All the details can be found in $[10,11,13]$. The result of the ADER predictor is a continuous second order space-time polynomial for both the numerical solution and the element geometry, which easily allows to evaluate any physical or geometric quantity in the space-time control volume $\omega_{c}(t)$ for any $t \in\left[t^{n} ; t^{n+1}\right]$. As a consequence, once the predictor is available, the subcell forces and the node values in (64)-(66) are simply fed with the high order extrapolated values of the predictor, hence for any variable it holds $\mathbf{q}^{*}(\boldsymbol{x})=\mathbf{q}_{h}\left(\boldsymbol{x}, t^{*}\right)$ for any space-time coordinate $(\boldsymbol{x}, t)$. 


\subsubsection{Space-time discretization of the left Cauchy-Green tensor equation}

The closure of the foregoing discrete system of conservation laws relies on the generic constitutive law (15) which requires the knowledge of the cell-centered left Cauchy-Green tensor $\mathbb{B}_{c}$ within the cell $\omega_{c}$. This is achieved by discretizing the geometrical conservation law (9) which is recalled hereafter for the sake of completeness

$$
\frac{\mathrm{d} \mathbb{B}}{\mathrm{d} t}-\mathbb{L} \mathbb{B}-\mathbb{B L}^{t}=0
$$

where $\mathbb{L}=\nabla \boldsymbol{v}$ is the Eulerian velocity gradient. In this paragraph, we aim at describing a second-order time discretization of this equation which is compatible with the grid displacement. Since by definition $\mathbb{B}=\mathbb{F F}^{t}$, let us investigate the approximation of the deformation gradient tensor, $\mathbb{F}$, over the Lagrangian cell $\Omega_{c}$ employing two complementary approaches. The first one relies on the introduction of the Finite Element approximation of the LagrangeEuler mapping at time $t>0$

$$
\begin{aligned}
\boldsymbol{\Phi}_{h}: \Omega_{c} & \rightarrow \omega_{c}(t) \\
\boldsymbol{X} & \mapsto \boldsymbol{x}_{h}(\boldsymbol{X}, t)=\sum_{p \in \mathcal{P}(c)} \lambda_{p}(\boldsymbol{X}) \boldsymbol{x}_{p}(t) .
\end{aligned}
$$

Since $\Omega_{c}$ and $\omega_{c}(t)$ are simplices, this mapping is well defined and the function $\lambda_{p}(\boldsymbol{X})$ is nothing but the barycenter coordinate of $\boldsymbol{X}$ related to point $p$. It is an affine function of $\boldsymbol{X}$ which is determined by solving $\lambda_{p}\left(\boldsymbol{X}_{q}\right)=\delta_{p q}$ for $q \in \mathcal{P}(c)$, where $\delta_{p q}$ is the Kroenecker symbol. The set of $\lambda_{p}$ functions for $p \in \mathcal{P}(c)$ constitutes the classical $\mathrm{P}_{1}$ Finite Element basis related to the simplex $\Omega_{c}$. Moreover, the Eulerian coordinate $\boldsymbol{x}_{p}$ is determined by solving the trajectory equation $\frac{d \boldsymbol{x}_{p}}{d t}=\boldsymbol{v}_{p}$ with $\boldsymbol{x}_{p}(0)=\boldsymbol{X}_{p}$. The deformation gradient associated to this Finite Element mapping is readily obtained

$$
\mathbb{F}_{h}=\frac{\partial \boldsymbol{x}_{h}(\boldsymbol{X}, t)}{\partial \boldsymbol{X}}=\sum_{p \in \mathcal{P}(c)} \boldsymbol{x}_{p}(t) \otimes \nabla_{X} \lambda_{p} .
$$

It is clear that in this framework the compatibility condition $\nabla_{X} \times \mathbb{F}_{h}=0$ is fulfilled by construction. Since this mapping is an affine function with respect to $\boldsymbol{X}$ the resulting deformation gradient is constant over the cell $\Omega_{c}$. Therefore, $\mathbb{F}_{h}$ represents a piecewise constant approximation of the deformation gradient. We observe that $\boldsymbol{x}_{h}(\boldsymbol{X}, 0)=\boldsymbol{X}$, thus $\mathbb{F}_{h}(\boldsymbol{X}, 0)=\mathbb{I}_{\mathrm{d}}$ and $J_{h}(\boldsymbol{X}, 0)=\operatorname{det}\left(\mathbb{F}_{h}(\boldsymbol{X}, 0)\right)=1$. We assume by a continuity argument that $J_{h}(\boldsymbol{X}, t)>0$ for all $t>0$, which ensures the integrity of the moving simplicial grid. Now, time differentiating the Finite Element mapping leads to the velocity field representation

$$
\boldsymbol{v}_{h}(\boldsymbol{X}, t)=\sum_{p \in \mathcal{P}(c)} \lambda_{p}(\boldsymbol{X}) \boldsymbol{v}_{p}(t)
$$

Subtracting the gradient of this velocity field from the time derivative of (73) leads to

$$
\frac{\partial \mathbb{F}_{h}}{\partial t}(\boldsymbol{X}, t)-\sum_{p \in \mathcal{P}(c)} \boldsymbol{v}_{p}(t) \otimes \nabla_{X} \lambda_{p}=0 .
$$

This shows that the foregoing Finite Element mapping provides a consistent spatial discretization of the kinematics which is fully compatible with the Geometrical Conservation 
Law (5).

On the other hand, applying the Green formula leads to introduce a cell-centered approximation of the deformation gradient over $\Omega_{c}$ as follows

$$
\mathbb{F}_{c}=\frac{1}{\left|\Omega_{c}\right|} \int_{\Omega_{c}} \nabla_{X} \boldsymbol{x} \mathrm{d} V=\frac{1}{\left|\Omega_{c}\right|} \int_{\partial \Omega_{c}} \boldsymbol{x} \otimes \boldsymbol{N} \mathrm{d} S .
$$

Approximating the second integral by means of the Lagrangian corner normal vector (39) yields

$$
\mathbb{F}_{c}=\frac{1}{\left|\Omega_{c}\right|} \sum_{p \in \mathcal{P}(c)} A_{p c} \boldsymbol{x}_{p} \otimes \boldsymbol{N}_{p c}
$$

Now, comparing the Finite Element approximation (73) to the Finite Volume approximation (75) shows that the Lagrangian corner normal $A_{p c} \boldsymbol{N}_{p c}$ is nothing but the gradient of the basis function $\lambda_{p}$ times the cell volume:

$$
\nabla_{X} \lambda_{p}(\boldsymbol{X})=\frac{A_{p c}}{\left|\Omega_{c}\right|} \boldsymbol{N}_{p c}
$$

This remarkable property could have been obtained directly differentiating the explicit expression of $\lambda_{p}$ with respect to $\boldsymbol{X}$, refer for instance to $[22,28]$. We claim that for simplicial grids the Finite Element approximation (73) and the Finite Volume one (75) of the deformation gradient coincide. Let us point out that the foregoing space discretization of the deformation gradient are expressed in terms of the Lagrangian grid. This would be relevant if we were working with a total Lagrangian formulation of the solid dynamics conservation laws. In our case, we are dealing with an updated Lagrangian formulation of these equations which requires a space discretization of the deformation gradient over the Eulerian grid. To this end, we shall express the Eulerian corner normal $a_{p c} \boldsymbol{n}_{p c}$ in terms of its Lagrangian counterpart thanks to the foregoing Finite Element mapping. Since the Eulerian corner normal $a_{p c} \boldsymbol{n}_{p c}$ is the gradient of the cell volume $\omega_{c}$ with respect to $\boldsymbol{x}_{p}$, there holds

$$
\mathrm{d}\left|\omega_{c}\right|=\sum_{p \in \mathcal{P}(c)} \frac{\partial\left|\omega_{p c}\right|}{\partial \boldsymbol{x}_{p}} \cdot \mathrm{d} \boldsymbol{x}_{p}=\sum_{p \in \mathcal{P}(c)} a_{p c} \boldsymbol{n}_{p c} \cdot \mathrm{d} \boldsymbol{x}_{p} .
$$

Recalling that $\boldsymbol{x}_{p}=\boldsymbol{x}_{p}(t)$ is the image of $\boldsymbol{X}_{p}$ through the Finite Element mapping, i.e., $\boldsymbol{x}_{p}(t)=\boldsymbol{x}_{h}\left(\boldsymbol{X}_{p}, t\right)$ and applying the chain rule leads to

$$
\mathrm{d} \boldsymbol{x}_{p}=\frac{\partial \boldsymbol{x}_{h}}{\partial \boldsymbol{X}}\left(\boldsymbol{X}_{p}, t\right) \mathrm{d} \boldsymbol{X}=\mathbb{F}_{h} \mathrm{~d} \boldsymbol{X}_{p}
$$

Substituting this expression of the differential of $\boldsymbol{x}_{p}$ into (77) and observing that $\left|\omega_{c}\right|=J_{h}\left|\Omega_{c}\right|$ yields

$$
J_{h} \mathrm{~d}\left|\Omega_{c}\right|=\sum_{p \in \mathcal{P}(c)} \mathbb{F}_{h}^{t} a_{p c} \boldsymbol{n}_{p c} \cdot \mathrm{d} \boldsymbol{X}_{p} .
$$

Knowing that the Lagrangian corner normal $A_{p c} \boldsymbol{N}_{p c}$ is the gradient of the Lagrangian volume $\Omega_{c}$ with respect to $\boldsymbol{X}_{p}$ we arrive at the relationship

$$
A_{p c} \boldsymbol{N}_{p c}=a_{p c} J_{c}^{-1} \mathbb{F}_{c}^{t} \boldsymbol{n}_{p c} .
$$

Here, for the deformation gradient and its Jacobian we have replaced the subscript $h$ by $c$ since $\mathbb{F}_{h}=\mathbb{F}_{c}$. 
Gathering the foregoing results the Lagrangian space discretization of the deformation gradient equation (74) rewrites

$$
\frac{\mathrm{d} \mathbb{F}_{c}}{\mathrm{~d} t}-\frac{1}{\left|\Omega_{c}\right|} \sum_{p \in \mathcal{P}(c)} A_{p c} \boldsymbol{v}_{p} \otimes \boldsymbol{N}_{p c}=0 .
$$

Expressing the Lagrangian corner normal in terms of the Eulerian one thanks to relationship (78) leads to

$$
\frac{\mathrm{d} \mathbb{F}_{c}}{\mathrm{~d} t}-\frac{1}{\left|\omega_{c}\right|} \sum_{p \in \mathcal{P}(c)} a_{p c}\left(\boldsymbol{v}_{p} \otimes \boldsymbol{n}_{p c}\right) \mathbb{F}_{c}=0 .
$$

Here, we have used the identity $\boldsymbol{a} \otimes \mathbb{Q}^{t} \boldsymbol{b}=(\boldsymbol{a} \otimes \boldsymbol{b}) \mathbb{Q}$ valid for any vectors $\boldsymbol{a}, \boldsymbol{b}$ and tensor $\mathbb{Q}$. Finally, recalling the definition of the discrete Eulerian velocity gradient operator, we arrive at the Eulerian space discretization of the deformation gradient equation

$$
\frac{\mathrm{d} \mathbb{F}_{c}}{\mathrm{~d} t}-\mathbb{L}_{c} \mathbb{F}_{c}=0
$$

where the Eulerian discrete velocity gradient operator is given by (40). This equation is the semi-discrete counterpart of the updated Lagrangian form of the deformation gradient equation (7). Let us emphasize the crucial role played by (78). This geometric relation allows us to maintain the consistency of the geometry of the initial grid with the geometry of the current grid. Indeed, thanks to (78), we should be able to construct consistent spatial discretization for both total and updated Lagrangian representations.

We construct the second-order time discretization of (79) employing the classical CrankNicholson integration scheme over the time interval $\left[t^{n}, t^{n+1}\right]$

$$
\mathbb{F}_{c}^{n+1}-\mathbb{F}_{c}^{n}-\frac{\Delta t}{2} \mathbb{L}_{c}^{n+\frac{1}{2}}\left(\mathbb{F}_{c}^{n+1}+\mathbb{F}_{c}^{n}\right)
$$

where $\mathbb{L}_{c}^{n+\frac{1}{2}}$ is the time-centered approximation of the discrete velocity gradient operator defined over $\omega_{c}$, which reads according to (40)

$$
\mathbb{L}_{c}^{n+\frac{1}{2}}=\frac{1}{\left|\omega_{c}^{n+\frac{1}{2}}\right|} \sum_{p \in \mathcal{P}(c)} a_{p c}^{n+\frac{1}{2}} \boldsymbol{v}_{p}^{n+\frac{1}{2}} \otimes \boldsymbol{n}_{p c}^{n+\frac{1}{2}}
$$

Here, the second-order space time discretization of the system of conservation laws achieved in section 3.4.1 thanks to the nodal solver (60) provides us the node velocity $\boldsymbol{v}_{p}^{n+\frac{1}{2}}$. Employing this time-centered kinematic velocity field allows us to integrate the trajectory equation up to second-order (63) and define the corresponding time-centered geometry which is labeled using the superscript $n+\frac{1}{2}$.

Performing the Taylor expansion $\operatorname{det}\left(\mathbb{I}_{\mathrm{d}}-\frac{\Delta t}{2} \mathbb{L}_{c}^{n+\frac{1}{2}}\right)=1-\frac{\Delta t}{2} \operatorname{tr}\left(\mathbb{L}_{c}^{n+\frac{1}{2}}\right)+O\left(\Delta t^{2}\right)$ for $\Delta t \rightarrow 0$ shows that we can find $\Delta t>0$ sufficiently small so that $\operatorname{det}\left(\mathbb{I}_{\mathrm{d}}-\frac{\Delta t}{2} \mathbb{L}_{c}^{n+\frac{1}{2}}\right)>0$ and thus $\mathbb{I}_{\mathrm{d}}-\frac{\Delta t}{2} \mathbb{L}_{c}^{n+\frac{1}{2}}$ is invertible. Under this time step constraint the updated cell-centered value of the deformation gradient within cell $\omega_{c}$ reads

$$
\mathbb{F}_{c}^{n+1}=\left(\mathbb{I}_{\mathrm{d}}-\frac{\Delta t}{2} \mathbb{L}_{c}^{n+\frac{1}{2}}\right)^{-1}\left(\mathbb{I}_{\mathrm{d}}+\frac{\Delta t}{2} \mathbb{L}_{c}^{n+\frac{1}{2}}\right) \mathbb{F}_{c}^{n}
$$


Let us point out that (80) is particularly interesting since it provides the time increment of the cell-centered deformation gradient employing uniquely geometrical quantities attached to the time-centered Eulerian grid. Now, taking the determinant of (80) yields

$$
\operatorname{det}\left(\mathbb{F}_{c}^{n+1}\right)=\frac{\operatorname{det}\left(\mathbb{I}_{\mathrm{d}}+\frac{\Delta t}{2} \mathbb{L}_{c}^{n+\frac{1}{2}}\right)}{\operatorname{det}\left(\mathbb{I}_{\mathrm{d}}-\frac{\Delta t}{2} \mathbb{L}_{c}^{n+\frac{1}{2}}\right)} \operatorname{det}\left(\mathbb{F}_{c}^{n}\right)
$$

Performing a Taylor expansion of the above fraction for $\Delta t \rightarrow 0$ up to second order leads to

$$
J_{c}^{n+1}=\left[1+\Delta t I_{1}\left(\mathbb{L}_{c}^{n+\frac{1}{2}}\right)+\frac{\Delta t^{2}}{2} I_{1}^{2}\left(\mathbb{L}_{c}^{n+\frac{1}{2}}\right)\right] J_{c}^{n}+O\left(\Delta t^{3}\right)
$$

where $I_{1}\left(\mathbb{L}_{c}^{n+\frac{1}{2}}\right)$ is the first invariant of the time-centered Eulerian velocity gradient, i.e., $I_{1}\left(\mathbb{L}_{c}^{n+\frac{1}{2}}\right)=\operatorname{tr}\left(\mathbb{L}_{c}^{n+\frac{1}{2}}\right)$. This formula has been obtained thanks to the use of the Taylor formula

$$
\operatorname{det}\left(\mathbb{I}_{\mathrm{d}}+h \mathbb{A}\right)=1+h I_{1}(\mathbb{A})+h^{2} I_{2}(\mathbb{A})+O\left(h^{3}\right), \text { when } h \rightarrow 0 .
$$

which holds true for any second-order tensor $\mathbb{A}$. Here, $I_{2}(\mathbb{A})$ is the second invariant of $\mathbb{A}$ whose definition is recalled in appendix A. This suggests the alternative time discretization of the Jacobian equation

$$
J_{c}^{n+1}=\left(1+\Delta t I_{1}+\frac{\Delta t^{2}}{2} I_{1}^{2}\right) J_{c}^{n}
$$

This is a positivity preserving time discretization since the quadratic polynomial between parenthesis is always strictly positive regardless $\Delta t$. It is also a second-order accurate approximation of

$$
J_{c}^{n+1}=J_{c}^{n} \exp \left[\Delta t \operatorname{tr}\left(\mathbb{L}_{c}^{n+\frac{1}{2}}\right)\right]
$$

which is nothing but the second-order time discretization of the GCL (8), i.e., $\frac{\mathrm{d} J}{\mathrm{~d} t}=J \operatorname{tr}(\mathbb{L})$. We observe that (81) could be employed in lieu and place of (64).

Finally, substituting (80) into the definition of the left Cauchy-Green tensor, i.e., $\mathbb{B}=\mathbb{F}^{t}$, we arrive at

$$
\mathbb{B}_{c}^{n+1}=\left(\mathbb{I}_{\mathrm{d}}-\frac{\Delta t}{2} \mathbb{L}_{c}^{n+\frac{1}{2}}\right)^{-1}\left(\mathbb{I}_{\mathrm{d}}+\frac{\Delta t}{2} \mathbb{L}_{c}^{n+\frac{1}{2}}\right) \mathbb{B}_{c}^{n}\left(\mathbb{I}_{\mathrm{d}}+\frac{\Delta t}{2} \mathbb{L}_{c}^{n+\frac{1}{2}}\right)^{t}\left(\mathbb{I}_{\mathrm{d}}-\frac{\Delta t}{2} \mathbb{L}_{c}^{n+\frac{1}{2}}\right)^{-t}
$$

Performing a Taylor expansion of the foregoing equation for $\Delta t \rightarrow 0$ allows to show that it constitutes a second-order accurate approximation of the GCL (9). Moreover, (82) is consistent with the discretization of the discrete Jacobian equation (81). The proposed time discretization preserves the symmetry and the positive definiteness of the left Cauchy-Green tensor. Indeed, denoting $\mathbb{Q}=\left(\mathbb{I}_{\mathrm{d}}-\frac{\Delta t}{2} \mathbb{L}_{c}^{n+\frac{1}{2}}\right)^{-1}\left(\mathbb{I}_{\mathrm{d}}+\frac{\Delta t}{2} \mathbb{L}_{c}^{n+\frac{1}{2}}\right)$ we get $\mathbb{B}_{c}^{n+1}=\mathbb{Q B}_{c}^{n} \mathbb{Q}^{t}$. Assuming $\mathbb{B}_{c}^{n}$ is symmetric and positive definite, it is clear that $\mathbb{B}_{c}^{n+1}$ remains symmetric. Regarding its positive definiteness it holds true since

$$
\mathbb{B}_{c}^{n+1} \boldsymbol{a} \cdot \boldsymbol{a}=\left(\mathbb{Q B}_{c}^{n} \mathbb{Q}^{t}\right) \boldsymbol{a} \cdot \boldsymbol{a}=\mathbb{B}_{c}^{n}\left(\mathbb{Q}^{t} \boldsymbol{a}\right) \cdot\left(\mathbb{Q}^{t} \boldsymbol{a}\right) \geq 0, \text { for any vector } \boldsymbol{a} .
$$




\subsection{Limiting: a posteriori MOOD loop}

While in the original ADER schemes the limiting relies on a priori limited WENO reconstructions for all variables [26,25], here we adopt an a posteriori MOOD paradigm, see $[21,13]$. The technique is a posteriori in the sense that we compute a solution at time $t^{n+1}$, and, then, determine if this candidate solution is acceptable, or not and some dissipation is needed. The candidate solution is first computed with a second-order accurate $\mathbb{P}_{1}$ unlimited scheme using a centered reconstruction stencil. Then a detection procedure determines the problematic cells, i.e. the cells where the approximation does not respect some user-given criteria. For those cells the solution is locally recomputed with a lower-order but more robust scheme. In this work we consider three schemes forming a cascade, each of them chosen to comply with one specific objective:

(1) $\mathbb{P}_{1}$ : Accuracy is gained with the unlimited piecewise-linear polynomial reconstruction: maximal second-order of accuracy, possibly oscillating;

(2) $\mathbb{P}_{1}^{\text {lim: }}$ Robustness is gained with the previous reconstruction supplemented with BarthJespersen (BJ) [3] slope limiter: between first- and second-order of accuracy, essentiallynon-oscillatory;

(3) $\mathbb{P}_{0}$ : Fail-safe is gained without any polynomial reconstruction: first-order of accuracy, robust but dissipative.

The cascade is then $\mathbb{P}_{1} \rightarrow \mathbb{P}_{1}^{\text {lim }} \rightarrow \mathbb{P}_{0}$, different from [13] which was reduced to $\mathbb{P}_{1} \rightarrow \mathbb{P}_{0}$. A cell which does not satisfy all detection criteria is recomputed with the next scheme in the cascade. This procedure, called the MOOD loop, is repeated until each cell satisfies all detection criteria or if the latest scheme of the cascade is selected. In this case, the ultrarobust first order FV parachute scheme is employed. Its role is to produce a meaningful solution at the price of an excessive numerical dissipation, but, in practice, it is almost never used. Notice that the BJ slope limiter can be substituted by any other reasonable one. The process of dropping in the cascade is called decrementing and a numerical solution not yet valid is referred to as being a candidate solution, see figure 3 for a sketch.

The efficiency of the a posteriori MOOD paradigm is brought by the fact that usually only few problematic cells need decrementing. In the present implementation, the MOOD loop simply embraces the main evolution routines of the ADER method and iterates to recompute those cells with invalid values, detected by the admissibility criteria. In the worst case scenario all cells in the domain are updated with the parachute scheme, leading to the true first-order accurate and robust numerical solution. On the other hand, in the best case scenario, all cells are admissible at the first MOOD iterate leading to a truly secondorder accurate numerical solution. In any other case, the MOOD loop always converges and produces an acceptable numerical solution, assuming that the parachute scheme does so.

In the case of hyperelasticity the detection/admissible criteria are based on the discrete version of $\mathcal{A}$, see remark 3 , that is, a candidate solution $\mathrm{Q}_{h}^{*, n+1}$ is physically admissible if it belongs to $\mathcal{A}_{h}=\left\{\mathbf{Q}_{c}=\left(\tau_{c}, \boldsymbol{v}_{c}, e_{c}, \mathbb{B}_{c}\right)\right.$ s.t. $\left.\tau_{c}>0, \varepsilon_{c}>0, \theta_{c}>0,\right\}$. Notice that we do not really use the entropy production in each cell, i.e. see (33), because it produces excessive dissipative numerical solutions without any apparent gain.

Moreover to avoid spurious oscillations we also demand that the candidate density fulfills a 
Relaxed Discrete Maximum Principle (RDMP) that is

$$
-\delta_{c}^{n}+m_{c}^{n} \leq \rho_{c}^{*, n+1} \leq M_{c}^{n}+\delta_{c}^{n}, \quad \text { with }\left\{\begin{array}{l}
\delta_{c}^{n}=\max \left(\delta_{0}, \delta_{1}\left|M_{c}^{n}-m_{c}^{n}\right|\right), \\
m_{c}^{n}=\min _{d \in \mathcal{V}_{c}}\left(\rho_{d}^{n}\right), \quad M_{c}^{n}=\max _{d \in \mathcal{V}_{c}}\left(\rho_{d}^{n}\right) .
\end{array}\right.
$$

$\mathcal{V}_{c}$ is the von Neumann neighborhood of cell $c$ used to reconstruct the piecewise polynomials. We fix $\delta_{0}=10^{-4}$ and $\delta_{1}=10^{-3}$. Any cell which does not belong to $\mathcal{A}_{h}$ or does not fulfill (83) is declared troubled, and, sent back to $t^{n}$ along with its neighbors for their re-computation using the next scheme in the cascade, see [13].

\subsection{Internal consistency consideration}

The internal consistency of the numerical scheme is related to its ability to maintain some mathematical equality at a reasonable level of accuracy during the simulation ${ }^{1}$.

The first of such equality is the link between the constant cell mass, the specific volume $\tau$ (or the density $\rho=1 / \tau$ ) and volume $|\omega|: m=\frac{|\omega|}{\tau}$. The discrete constant mass is computed initially from the initial specific volume in the cell as $m_{c} \equiv m_{c}^{0}=\frac{\left|\omega_{c}^{0}\right|}{\tau_{c}^{0}}$. The primary variable is the specific volume: $\tau_{c}^{n+1}$ computed by (64). The new position of the points $\boldsymbol{x}_{p}^{n+1}$ allows to deduce the cell volume $\left|\omega_{c}^{n+1}\right|$ as a secondary variable. However, we could also deduce the cell volume consistently with the PDE (49) as being $\tau_{c}^{n+1} m_{c}$. Therefore, from a consistency point of view, we should monitor that the following equality holds

$$
\epsilon_{\omega}=|| \omega_{c}^{n+1}\left|-\tau_{c}^{n+1} m_{c}\right|
$$

This constraint is nothing but the discrete version of the GCL which is fulfilled by construction for modern cell-centered Lagrangian schemes thanks to the exact integration of the geometry in (67).

For the hyperelasticity model, the identity $\operatorname{det} \mathbb{B}=J^{2}=\left(\frac{\tau^{0}}{\tau}\right)^{2}$ should be fulfilled throughout the simulation. For each cell, numerically, $\tau_{c}^{n+1}$ is a primary variable computed from (64), and, a priori, not directly related to $\tau_{c}^{0} / \sqrt{\operatorname{det} \mathbb{B}_{c}^{n+1}}$. We therefore monitor their difference as an internal consistency criteria as

$$
\epsilon_{\mathbb{B}}=\left|\sqrt{\operatorname{det} \mathbb{B}_{c}^{n+1}}-\frac{\tau_{c}^{0}}{\tau_{c}^{n+1}}\right|
$$

Here $\epsilon_{\mathbb{B}}$ must be small compared to the accuracy of the scheme, and, in fact, the current scheme ensures that $\epsilon_{\mathbb{B}}=\mathcal{O}\left(\Delta t^{3}\right)$ due to the use of Simpson's rule in (67), see section 5.9 for a numerical validation.

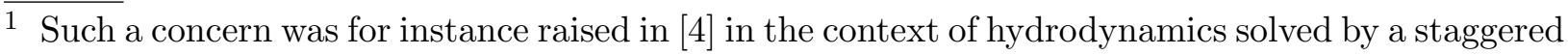
Lagrangian scheme where the cell volume can be computed either from the point coordinates or the PDE for the specific volume $\tau$. The difference between these two "measures" was monitored to assess the internal consistency of the scheme. 


\subsection{Time-step monitoring}

The time-step is restricted by the classical CFL condition in our Lagrangian context [44]

$$
\Delta t=\min \left(\Delta t_{\text {volume }}, \Delta t_{\text {acoustic }}, \Delta t_{\text {increase }}\right)
$$

where we have used a criterion to avoid a too large increase of cell volume in a single time-step

$\Delta t_{\text {vol. }}=C_{v} \min _{c}\left(\frac{\left|\omega_{c}^{n}\right|}{\sum_{p \in \mathcal{P}(c)} a_{p c} \boldsymbol{n}_{p c} \cdot \boldsymbol{v}_{p}}\right), \Delta t_{\text {acoust. }}=C_{\mathrm{CFL}} \min _{c}\left(\frac{L_{c}}{z_{c} / \rho_{c}}\right), \Delta t_{\text {incr. }}=C_{i}\left(t^{n}-t^{n-1}\right)$,

where $L_{c}$ is the cell characteristics length (smallest diameter of the in-spheres) and $z_{c} / \rho_{c}=$ $\sqrt{a_{c}^{2}+\frac{4}{3} \frac{\mu_{c}}{\rho_{c}}}$, and, $\left\{C_{v}, C_{\mathrm{CFL}}, C_{i}\right\} \in[0,1]^{3}$. The last constraint is designed to avoid a too large increase of $\Delta t$. Notice that the a posteriori detection allows to ensure the positivity of the cell volume and the internal energy provided that the parachute first-order scheme does. As such the time-step control must be suited for the parachute scheme. In our simulations we take $C_{v}=0.2, C_{i}=0.1$ and $C_{\mathrm{CFL}}=0.25$ otherwise noticed.

Notice that the a posteriori MOOD loop may also be used to try to exceed the time-step restrictions (86) at the price of creating more troubled cells, for instance by setting $C_{\mathrm{CFL}}$ closer to one.

\subsection{Boundary condition treatments}

The Boundary Conditions (BCs) play a crucial role in the time evolution of the numerical solution. In the context of an hyperelasticity model solved by the Lagrangian numerical scheme we consider several types of BCs, such as free traction, restricted normal/tangential displacement and contact/symmetry plans. These classical BCs are described in appendix B in the context of hyperelastic materials, and all are applied through the nodal solver, differently from other face-based FV schemes.

To enlarge even further the ability of the code to handle complex situations, we have added the possibility for a BCs to change its type during the simulation, for instance transitioning from free-traction to null normal velocity. Generally such $\mathrm{BC}$ type evolution is driven by the nullification of a cost or distance function $\mathcal{D}$. For instance an elastic material balistically flying, impacting onto a wall, spreading and eventually detaching, demands such type of evolving BCs, see for instance the test case 'Rebound of a hollow bar' in section 5.6.

The transition from $\mathrm{BC}$ type $A\left(\mathrm{BC}_{A}\right)$ to $B\left(\mathrm{BC}_{B}\right)$ can be imposed in two different ways:

- at a prescribed instant $t=t_{B C}$ the type of $\mathrm{BCs}$ changes, hence $\mathrm{BC}_{A} \rightarrow \mathrm{BC}_{B}$;

- when the moving medium approaches a prescribed target located at $\boldsymbol{x}_{T}$, i.e. the distance function $\mathcal{D}=\left|\boldsymbol{x}_{p}-\boldsymbol{x}_{T}\right|<\epsilon_{\mathcal{D}}$, where $\epsilon_{\mathcal{D}}$ is a user-given threshold value, and, the velocity vector points in the direction of the target, then $\mathrm{BC}_{A} \rightarrow \mathrm{BC}_{B}$. Later, if the medium happens to detach from the target, then the distance function becomes again greater than the threshold value and the original $\mathrm{BC}$ is restored, that is $\mathrm{BC}_{B} \rightarrow \mathrm{BC}_{A}$.

Finally, from a practical point of view a hierarchy between the type of BCs must be imposed. For instance when two faces sharing the same node must fulfill two different types of BCs, 
then they must be applied in a hierarchical manner, taking into account the most important one first, possibly relaxing the fulfillment of the other ones. Also at a material corner, a wall type BC must prevail compared to free traction BC, in such a way it avoids the boundary node to penetrate into the wall line/plane. Our hierarchy is as follows: 1- wall BC (restricted normal/tangential displacement), 2- space-dependent BC on velocity or pressure, 3- symmetry BC, and 4- free-traction BC.

Although it seems at first glance to be "only" implementation issues, the treatment of BCs is of utmost importance for 3D moving mesh numerical schemes.

\section{Implementation considerations}

\subsection{Algorithm}

In this section we recall the main steps of the MOOD loop applied to this cell-centered Lagrangian scheme sketched in figure 3. First of all, cell-centered unlimited polynomials of degree $d=1$ are reconstructed for any cell $c$ starting from data at $t^{n}, \mathbf{Q}_{c}^{n}$. Then a nodal solver and the ADER methodology allow to compute a candidate solution at $t^{n+1}$, leading to an $\mathbb{P}_{1}$ (unlimited 2nd order accurate) candidate solution. This candidate solution in cell $c$ can be either acceptable or numerically/physically wrong. This is the purpose of the 'Detection' box to determine which cells are troubled, and, on the contrary to accept the admissible ones. For those troubled cells, the 'Decrement' box picks the next scheme in the 'cascade', that is either one employing a $\mathbb{P}_{1}^{\text {lim }}$ piecewise-limited reconstruction (BJ limiter), or, $\mathbb{P}_{0}$ (no reconstruction at all, $d=0$ ), i.e. the parachute scheme. Those troubled cells and their Voronoi neighbors are solely sent back into the Lagrangian FV solver for re-computation. This part of the solution which has been recomputed is re-tested against the detection criteria. New admissible cells are accepted, while troubled ones are again sent back for re-computation with a more robust scheme. Notice that this MOOD loop converges in a finite number of steps because the number of schemes in the cascade is fixed as well as the number of cells.

Once the slope limiter is chosen, the only parameters of the numerical method are the thresholds $\delta_{0}$ and $\delta_{1}$ in (83) and the time-step control parameters (87).

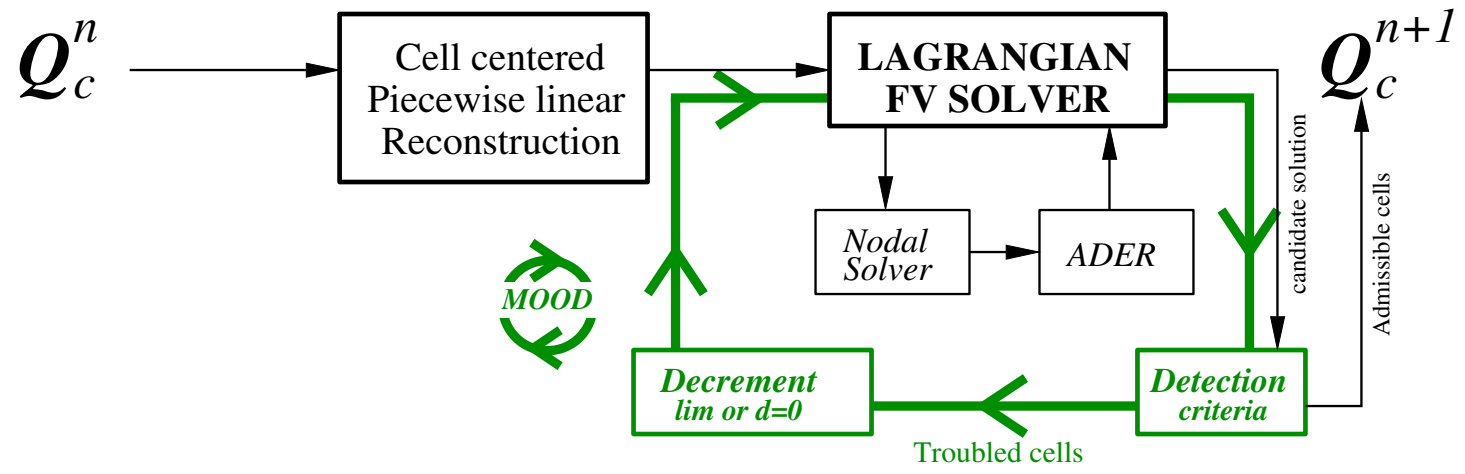

Fig. 3. Sketch of the current Lagrangian numerical method and its associated MOOD loop. 


\subsection{Meshing and parallelization}

The 3D Lagrangian simulation algorithm is fully coded in FORTRAN and relies on MPI protocol for the parallelization and the free graph partitioning software METIS [38]. More precisely the computational domain $\omega \in \mathbb{R}^{3}$ is first meshed with a genuinely coarse mesh made of large tetrahedra, say $N_{C}$, using any classical 3D mesh generator. $N_{C}$ is chosen small enough for the resulting coarse mesh to be handled by one processor without any difficulty. This primary mesh is then partitioned among the total number of threads $N_{\mathrm{CPU}}$, see figure 4right for $N_{\mathrm{CPU}}=4$ in 2D and the coarse mesh in black. Each MPI rank locally refines its portion of the primary mesh by an arbitrary refinement factor $\ell>0$.

$N_{\mathrm{CPU}}$ and $\ell>0$ are given by the user. A local isotropic recursive refinement is further applied. The $\ell=0$ th level corresponds to one of the primary tetrahedron, that is $N_{\ell}=1$ cell. The $\ell=1$ st level consists of its division into eight sub-tetrahedra, see remark 4 , to get $N_{\ell}=8$ sub-tetrahedra. The $\ell$ th level consists of the division of all sub-tetrahedra obtained at level $\ell-1$, leading to $N_{\ell}=8 \ell$ sub-tetrahedra. In $2 \mathrm{D}$ the subdivision of one triangle is made into 4 sub-triangles. Each thread possesses only a portion of the full mesh and writes also its own output files. As such the full mesh is never really assembled on a single thread leading to a reduction of memory storage. Notice that our aim is not to produce a perfectly scaled and massively parallel code, but rather being able to simulate with large enough 3D mesh reasonably fast. Nonetheless, a strong scaling up to 64 CPUs is shown in table 1, where we report the computational time $\tau_{E U}$ needed for performing one element update at second order of accuracy and the corresponding speedup for the L-shaped test case from section 5.8.

Table 1

\begin{tabular}{r|c|cc}
$\mathrm{CPU}$ & $\tau_{E U}$ & Measured speedup & Ideal speedup \\
\hline 8 & $2.30714 \mathrm{E}-05$ & 1.00 & 1 \\
16 & $1.28105 \mathrm{E}-05$ & 1.80 & 2 \\
32 & $6.29876 \mathrm{E}-06$ & 3.66 & 4 \\
64 & $2.99938 \mathrm{E}-06$ & 7.69 & 8
\end{tabular}

Strong scaling computed for the L-shaped block test case presented in section 5.8. The computational time needed to perform an element update with second order of accuracy in space and time is addressed with $\tau_{E U}$.

Remark 4 To split one single tetrahedron we insert new vertices at the midpoints of each edge and connect the vertices together and form four new sub-tetrahedra associated to the vertices. When removed, it remains one central octahedron which can further be split into four more sub-tetrahedra by arbitrarily choosing an octahedron diagonal, see figure 4-left.

\section{$5 \quad 2 \mathrm{D}$ and $3 \mathrm{D}$ test problems}

In the following we present the results for a set of $2 \mathrm{D}$ and $3 \mathrm{D}$ benchmark test cases. We refer to the current Lagrangian ADER-MOOD method with the acronym 'LAM'. Moreover two MOOD cascades are employed: $\mathbb{P}_{1} \rightarrow \mathbb{P}_{0}$, denoted as "LAM $\mathbb{P}_{0}$-lim" and $\mathbb{P}_{1} \rightarrow \mathbb{P}_{1}^{\text {lim }} \rightarrow \mathbb{P}_{0}$ 

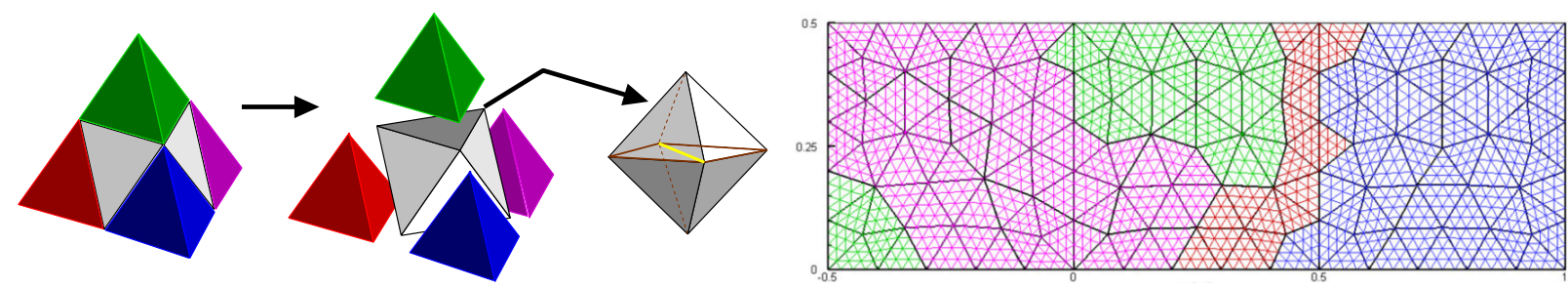

Fig. 4. Left: Split of a tetrahedron into sub-tetrahedra by inserting six new midpoint edge vertices to get four corner sub-tetrahedra (colored ones). After choosing a diagonal (yellow line) to split the remaining central octahedron into fours more sub-tetrahedra, it yields a total of eight sub-tetrahedra - Right: example of $2 \mathrm{D}$ partitioning on $N_{\mathrm{CPU}}=4$ threads (colors), the refinement is performed locally to each thread, only the coarse partition of large black triangles is actually built across the threads.

denoted "LAM $\mathbb{P}_{1}$-lim". The first cascade was employed for the hydrodynamics equations in [13], the second one is tested in this work. For each test problem the CFL stability coefficient is assumed to be 0.4 in $2 \mathrm{D}$ and 0.25 in $3 \mathrm{D}$. The time-dependent computational domain is addressed with $\omega(t)$, while $\mathbf{Q}(\boldsymbol{x}, t=0) \equiv \mathbf{Q}_{0}(\boldsymbol{x})=\left(1 / \rho_{0}, \boldsymbol{v}_{0}, p_{0}, \mathbb{B}_{0}\right)$ denotes the vector of initial conservative variables typically used to setup the test problems. $\mathbb{B}_{0}$ is set to the identity matrix as we only consider initially unloaded materials. The unstructured tetrahedral meshes are obtained by meshing software, such as GMSH [32] which takes a characteristics target length $h$ as input parameter.

According to [36], in order to highlight the advantages of adding a second order limited scheme in the cascade compared to a first order discretization, the numerical dissipation $\boldsymbol{\delta}$ is monitored and here evaluated as

$$
\boldsymbol{\delta}=\frac{\Psi+k-E_{0}}{E_{0}},
$$

with the kinetic and total energy at the initial time defined by

$$
k_{0}=\frac{1}{2} \boldsymbol{v}_{0}^{2}, \quad E_{0}=\Psi_{0}+k_{0}
$$

Finally, if not stated otherwise, the simplified neo-Hookean equation of state (27) is adopted, while in the last test the stiffened gas EOS (28) is used.

\subsection{Swinging plate}

The 2D swinging plate test problem, see $[40,53]$, is employed to evaluate the numerical order of convergence. The computational domain is $\omega=[0,2]^{2}$ and the analytical solution for the velocity is given by

$$
\boldsymbol{v}^{e x}(\boldsymbol{x}, t)=\omega U_{0} \cos (\omega t)\left(\begin{array}{c}
-\sin \left(\frac{\pi}{2} x\right) \cos \left(\frac{\pi}{2} y\right) \\
\cos \left(\frac{\pi}{2} x\right) \sin \left(\frac{\pi}{2} y\right)
\end{array}\right), \quad \omega=\frac{\pi}{2} \sqrt{\frac{2 \mu}{\rho^{0}}}
$$

with $U_{0}=5 \cdot 10^{-4} \mathrm{~m}$. The material under consideration is characterized by $\rho^{0}=1100 \mathrm{~kg} \cdot \mathrm{m}^{-3}$ with Young's modulus $E=1.7 \cdot 10^{7} \mathrm{~Pa}$ and Poisson ratio $\nu=0.45$. The velocity and displacement fields are divergence-free, leading to the exact pressure $p^{e x}=0$. Space-time 
dependent boundary conditions are prescribed for the normal velocities, according to the exact solution (89). Notice that the exact solution is a smooth one and the final time is set to $t_{\text {final }}=\pi / \omega$, and the final displacement corresponds to the initial one. In table 2 we report the $L_{2}$ errors $\epsilon$ at the final time for the horizontal velocity $u$, the first component of the left Cauchy-Green tensor $\mathbb{B}_{11}$ and of the Cauchy stress tensor $\mathbb{T}_{11}$. The unstructured mesh made of triangles is successively refined and the final characteristics length $L_{c}\left(\omega\left(t_{\text {final }}\right)\right)$ is measured and further used to compute the numerical order of convergence $\mathcal{O}$ as reported in table 2, where one can notice that the numerical scheme is able to retrieve the second-order of convergence on this regular solution.

Table 2

\begin{tabular}{|c|cc|cc|cc|}
\hline$L_{c}\left(\omega\left(t_{\text {final }}\right)\right)$ & $\epsilon(u)$ & $\mathcal{O}(u)$ & $\epsilon\left(\mathbb{B}_{11}\right)$ & $\mathcal{O}\left(\mathbb{B}_{11}\right)$ & $\epsilon\left(\mathbb{T}_{11}\right)$ & $\mathcal{O}\left(\mathbb{T}_{11}\right)$ \\
\hline \hline $7.81 \mathrm{E}-02$ & $2.144 \mathrm{E}-03$ & - & $1.581 \mathrm{E}-04$ & - & $9.681 \mathrm{E}+02$ & - \\
$5.21 \mathrm{E}-02$ & $8.206 \mathrm{E}-04$ & 2.37 & $7.072 \mathrm{E}-05$ & 1.98 & $4.258 \mathrm{E}+02$ & 2.03 \\
$3.91 \mathrm{E}-02$ & $4.650 \mathrm{E}-04$ & 1.97 & $3.914 \mathrm{E}-05$ & 2.06 & $2.343 \mathrm{E}+02$ & 2.08 \\
$3.13 \mathrm{E}-02$ & $3.085 \mathrm{E}-04$ & 1.84 & $2.473 \mathrm{E}-05$ & 2.06 & $1.477 \mathrm{E}+02$ & 2.07 \\
$2.60 \mathrm{E}-02$ & $2.212 \mathrm{E}-04$ & 1.82 & $1.699 \mathrm{E}-05$ & 2.06 & $1.015 \mathrm{E}+02$ & 2.06 \\
\hline \multicolumn{2}{|c|}{ Expected orders $\rightarrow$} & 2 & & 2 & & 2 \\
\hline
\end{tabular}

Numerical errors in $L_{2}$ norm and convergence rates for the $2 \mathrm{D}$ swinging plate test computed with second order of accuracy Lagrange ADER scheme at time $t_{\text {final }}=\pi / \omega$. The error norms refer to the variables $u$ (horizontal velocity), $\mathbb{B}_{11}$ (first component of the left Cauchy-Green tensor $\mathbb{B}$ ) and $\mathbb{T}_{11}$ (first component of the Cauchy stress tensor $\mathbb{T}$ ).

\subsection{Elastic vibration of a Beryllium plate}

This test case describes the elastic vibration of a beryllium plate or bar, see $[49,14]$ for instance. Here we consider the $2 \mathrm{D}$ version, that is the vibration of a plate. The computational domain is $\omega(t=0)=[-0.03,0.03] \times[-0.005,0.005]$ of length $L=0.06 \mathrm{~m}$. The material under investigation is characterized by $\rho^{0}=1845 \mathrm{~kg} \cdot \mathrm{m}^{-3}, E=3.1827 \cdot 10^{11} \mathrm{~Pa}$ and $\nu=0.0539$. The material is initially loaded via a perturbed initial velocity field $\boldsymbol{v}^{0}=\left(0, v^{0}(x)\right)$ of the form

$$
v^{0}(x)=A \omega\left[a_{1}\left(\sinh \left(x^{\prime}\right)+\sin \left(x^{\prime}\right)\right)-a_{2}\left(\cosh \left(x^{\prime}\right)+\cos \left(x^{\prime}\right)\right)\right],
$$

where $x^{\prime}=\alpha(x+L / 2), \alpha=78.834 \mathrm{~m}^{-1}, A=4.3369 \times 10^{-5} \mathrm{~m}, \omega=2.3597 \times 10^{5} \mathrm{~s}^{-1}$, $a_{1}=56.6368$ and $a_{2}=57.6455$. The final time is $t_{\text {final }}=3 \cdot 10^{-5} \mathrm{~s}$, see figure 5 for a sketch. Free boundary conditions are applied on the plate faces. The unstructured triangulation is constituted of $N_{c}=5344$ cells. In figure 6 we present the numerical results obtained at different output times for the pressure (left panels) and cell orders (right panels). The pressure field is coherent with results from the literature. On the right panel we plot the cell order, which is equivalent to record which scheme from the cascade is actually employed. Yellow cells are dealt with the unlimited second order scheme (maximal order, prone to oscillation), while the blue ones employ a piecewise reconstruction limited by BJ slope limiter, via the a posteriori MOOD loop. For this relatively mild problem, no cell is updated with 

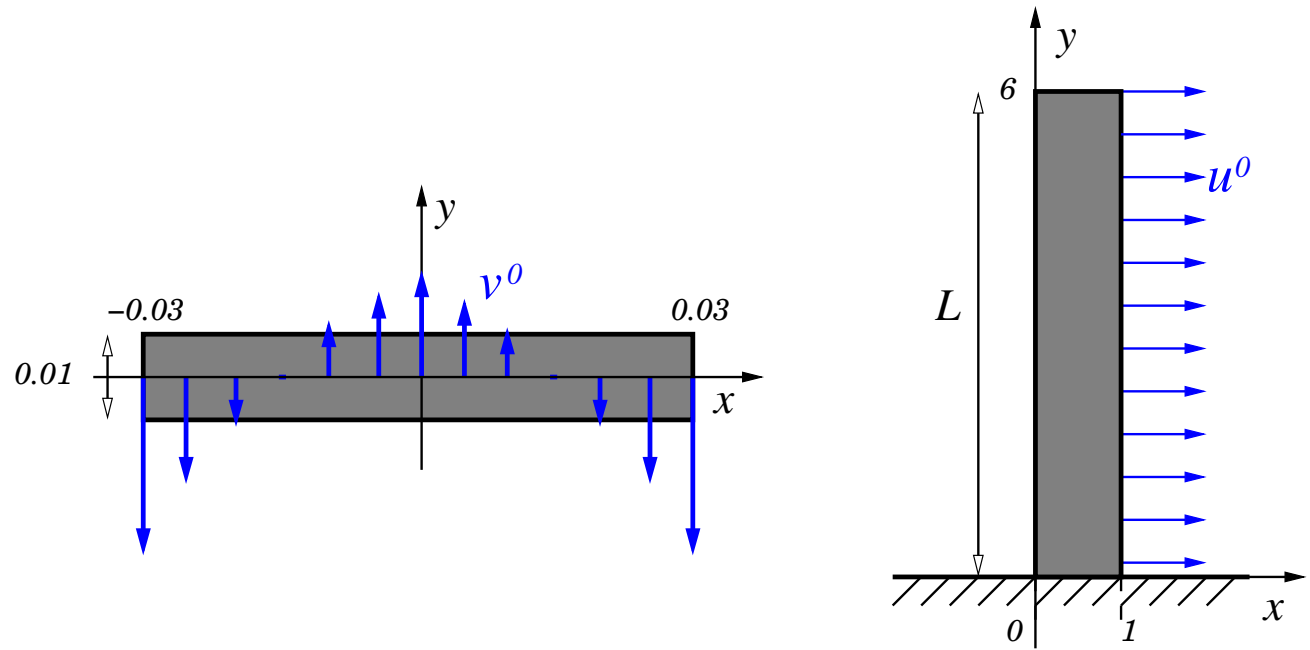

Fig. 5. Sketch for the elastic vibration of a beryllium plate in section 5.2 (left) and the finite deformation of a cantilever thick beam in section 5.3 (right).

the parachute scheme. Moreover no spurious modes nor artificial oscillations are observed.
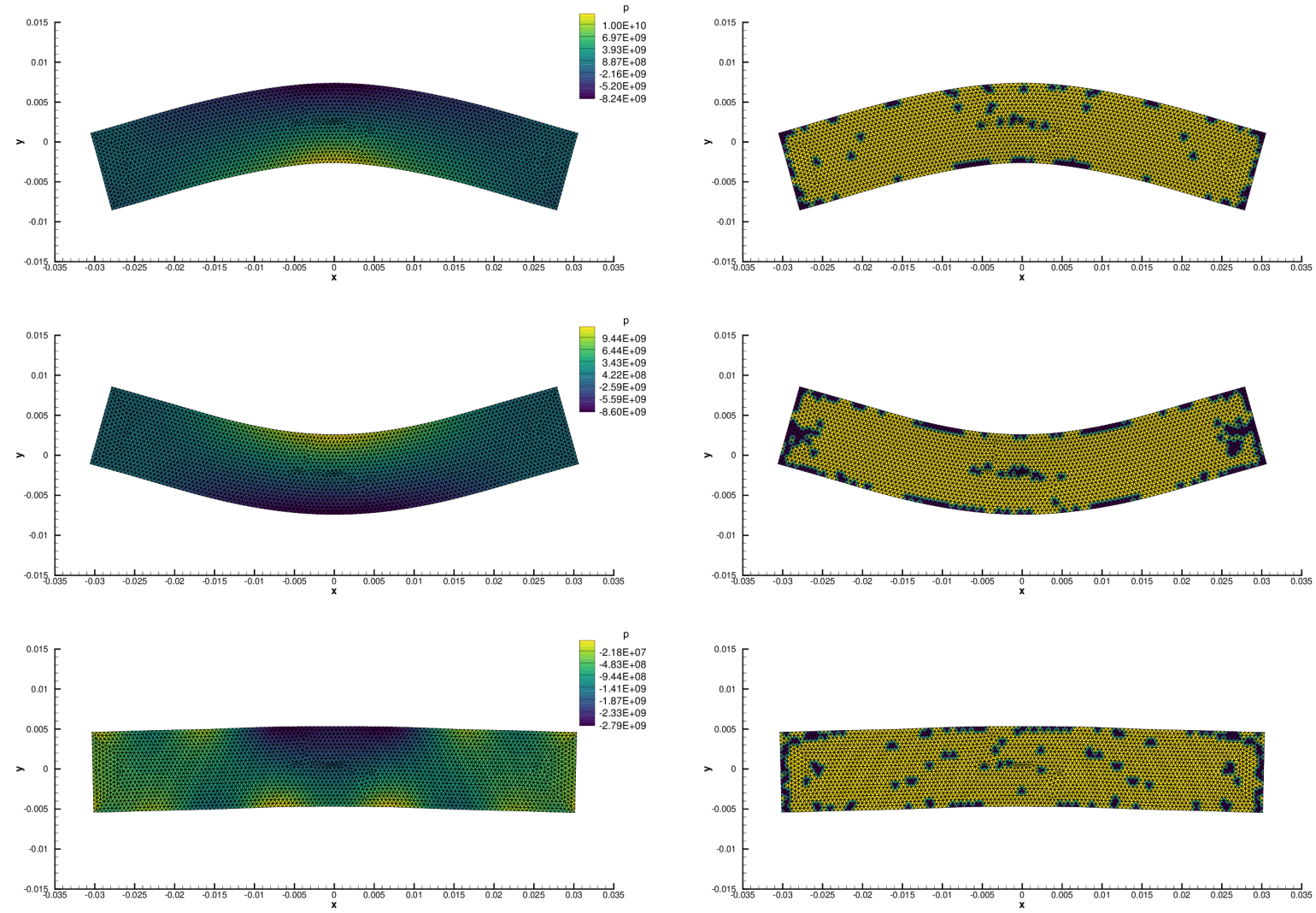

Fig. 6. Elastic vibration of a beryllium plate - Numerical results at output times $t=10^{-5}$ (top), $t=2 \cdot 10^{-5}$ (middle) and $t=3 \cdot 10^{-5}$ (bottom) for pressure (left) and cell order map (right), the cells in yellow are at unlimited order 2 , while the blue ones are the BJ limited ones. No first-order updated cell is observed.

In order to illustrate the reduction of dissipation when the cascade is not $\mathbb{P}_{1} \rightarrow \mathbb{P}_{0}$, like in [13], but $\mathbb{P}_{1} \rightarrow \mathbb{P}_{1}^{\text {lim }} \rightarrow \mathbb{P}_{0}$ instead, we show in figure 7 two diagnostics. First, on the left panel, the vertical displacement at the barycenter of the plate as a function of time is presented for the two cascades. As expected the nominally second order scheme is able 
to follow the barycenter with lower dissipation. On the right panel we enhance the actual numerical dissipation computed with (88) which confirms that a high order scheme reduces the numerical viscosity by about $75 \%$ at final time.
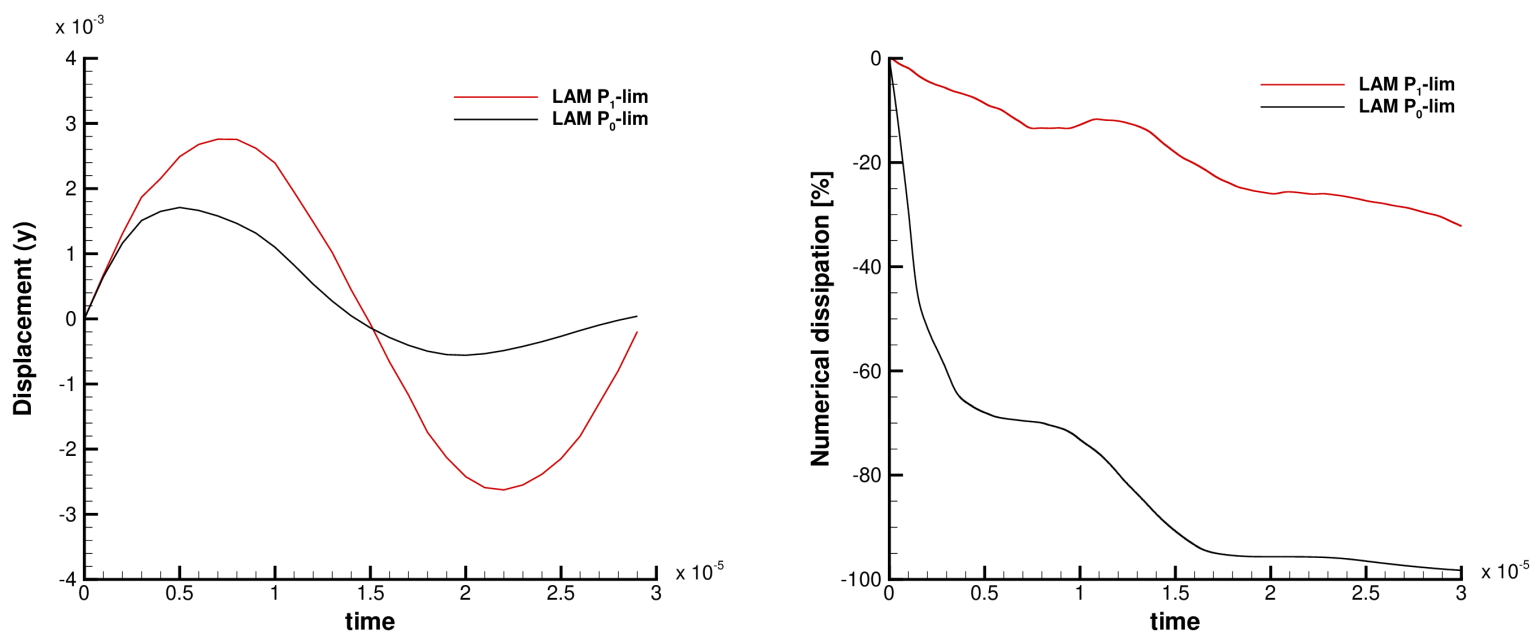

Fig. 7. Elastic vibration of a beryllium plate - Comparison between the MOOD cascades: $\mathbb{P}_{1} \rightarrow \mathbb{P}_{0}$ $\left(\mathrm{LAM} \mathbb{P}_{0}\right.$-lim) and $\mathbb{P}_{1} \rightarrow \mathbb{P}_{1}^{\text {lim }} \rightarrow \mathbb{P}_{0}$ (LAM $\mathbb{P}_{1}$-lim) for the vertical displacement at the barycenter of the plate (left) and the computed numerical dissipation (88) as a function of time (right).

\subsection{Finite deformation of a cantilever thick beam}

In [40] the authors present a test case involving a finite deformation of a $2 \mathrm{D}$ cantilever vertical thick beam of length $L$ having a unit square cross section and initially loaded by a uniform horizontal velocity $u^{0}=10 \mathrm{~m} . \mathrm{s}^{-1}$ whilst the unit width base is maintained fixed, see figure 5 for a sketch. We consider the initial computational domain $\omega(t=0)=[0 ; 1] \times[0 ; 6]$ leading to $L=6 \mathrm{~m}$ and material characteristics $\rho^{0}=1100 \mathrm{~kg} \cdot \mathrm{m}^{-3}, E=1.7 \cdot 10^{7} \mathrm{~Pa}$ and $\nu=0.45$. Free boundary conditions are considered apart from the fixed-wall bottom of the bar. The mesh is made of $N_{c}=5442$ triangles. The simulations are run with the cascade $\mathbb{P}_{1} \rightarrow \mathbb{P}_{1}^{\lim } \rightarrow \mathbb{P}_{0}$. On the left panels of figure 8 , we present the pressure distribution along with the deformed shapes at four different output times. The results are qualitatively in agreement with the published ones from the literature. Moreover we observe on the right panels that the yellow cells (unlimited second-order scheme) are massively represented, while only few of them demand dissipation (blue cells). For comparison purposes we also superimpose in black line the shapes obtained with the simpler cascade $\mathbb{P}_{1} \rightarrow \mathbb{P}_{0}$ from [13]. As can be observed, this latter scheme is genuinely more dissipative, and it numerically justifies the need for using a second order limited reconstruction within the cascade.

Then in figure 9 we show the computed numerical dissipation (88) as a function of time for the two cascades, where about $60 \%$ less dissipation is obtained by the current scheme with $\mathbb{P}_{1} \rightarrow \mathbb{P}_{1}^{\text {lim }} \rightarrow \mathbb{P}_{0}$ cascade. At last the right panel presents the percentage of troubled cells encountered as a function of time. On average about $5 \%$ of cells are re-computed at each time step. 

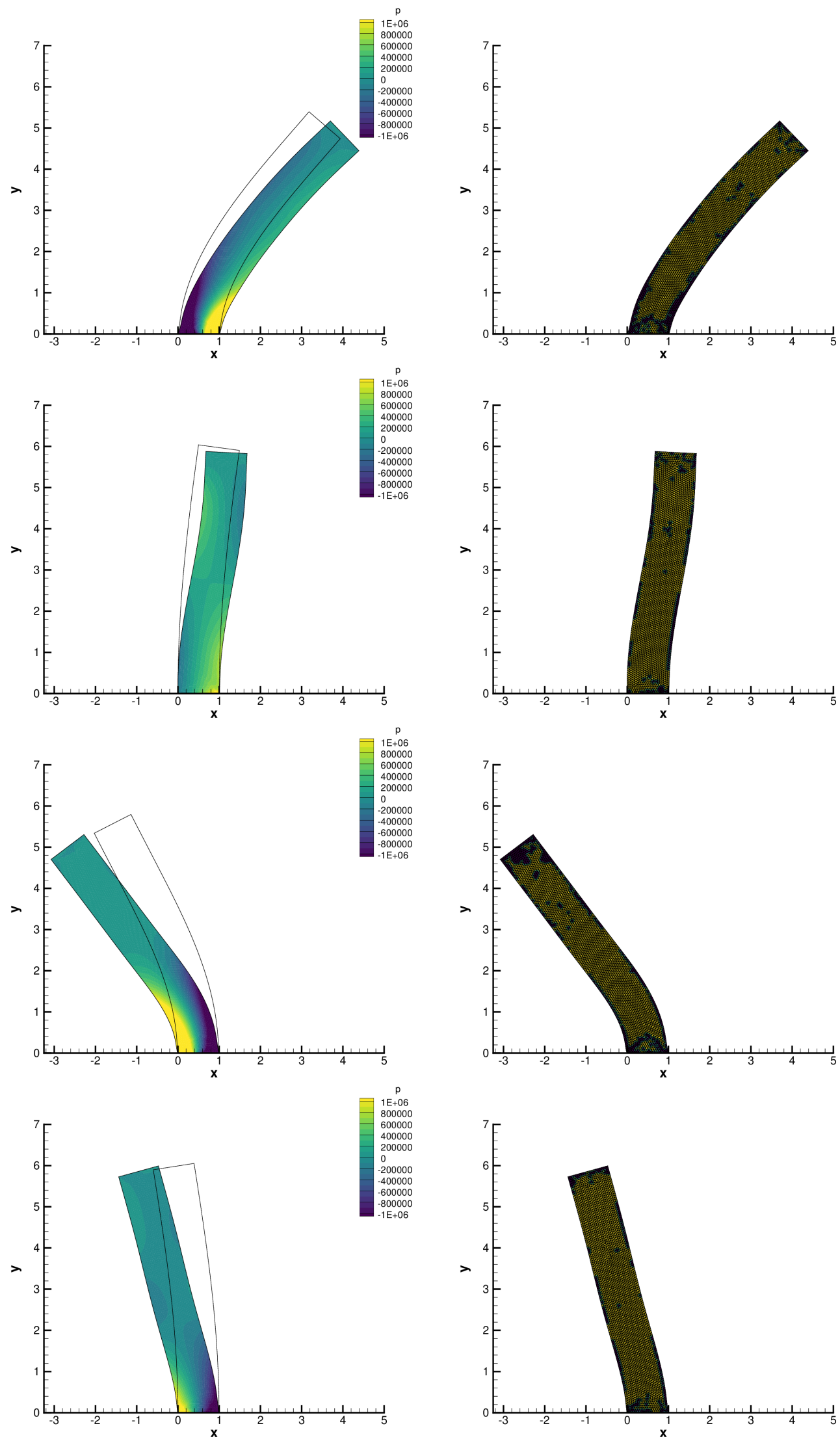

Fig. 8. Cantilever thick beam test case - Pressure distribution with deformed shapes (left column) and cell order map (right column) with the second-order a posteriori limited Lagrangian scheme at output times $t=0.375, t=0.75, t=1.125$ and $t=1.5$ (from top to bottom row) - Comparison of the deformed shape computed using the simpler cascade $\mathbb{P}_{1} \rightarrow \mathbb{P}_{0}$ in black line on the left panels only. 

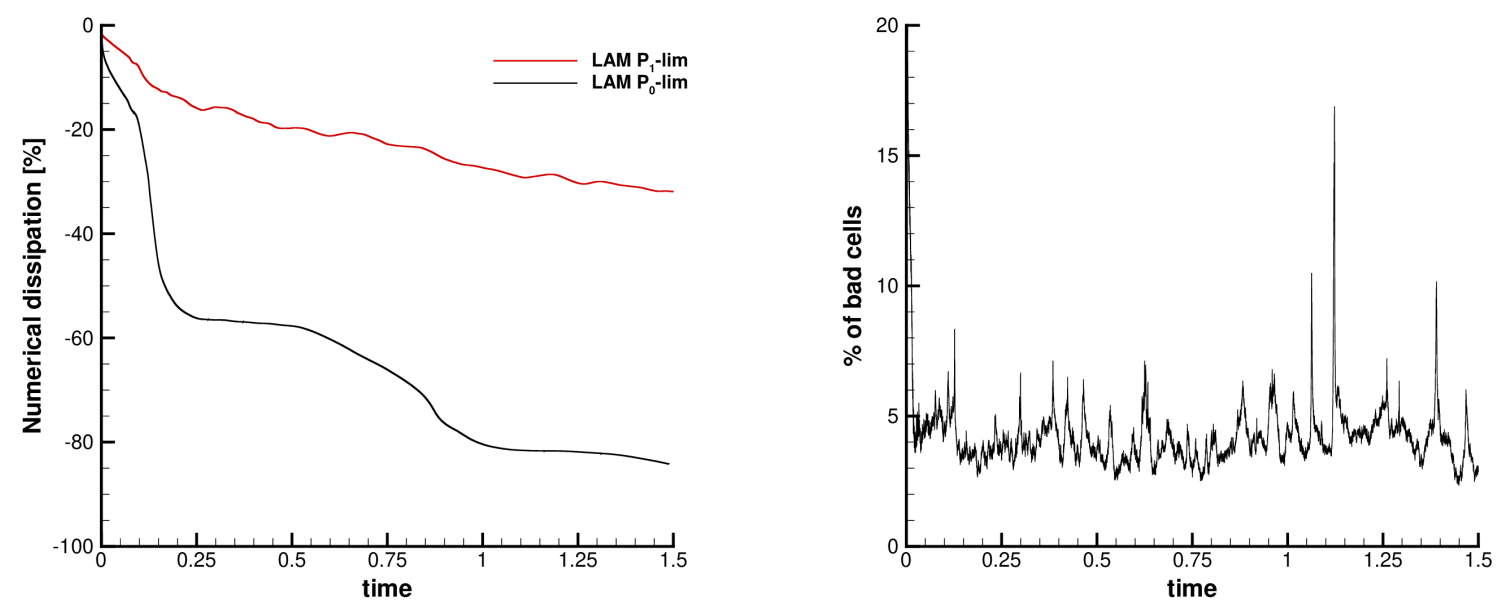

Fig. 9. Cantilever thick beam - Comparison between the MOOD cascades: $\mathbb{P}_{1} \rightarrow \mathbb{P}_{0}\left(\mathrm{LAM} \mathbb{P}_{0}-\mathrm{lim}\right)$ and $\mathbb{P}_{1} \rightarrow \mathbb{P}_{1}^{\lim } \rightarrow \mathbb{P}_{0}$ (LAM $\mathbb{P}_{1}$-lim) for the computed numerical dissipation (88) as a function of time (left) and percentage of bad cells detected at each time step (right).

\subsection{Blake's problem}

Blake's problem is a classical spherical test derived from the small strain linear elasticity theory [37]. The domain is a shell of inner radius $r_{\text {in }}=0.1 \mathrm{~m}$ and outer radius $r_{\text {out }}=1 \mathrm{~m}$. The shell material is isotropic with parameters: $\rho_{0}=3000 \mathrm{~kg} \cdot \mathrm{m}^{-3}$, Young's modulus $E=$ $62.5 \cdot 10^{9} \mathrm{~Pa}$ and Poisson's ratio $\nu=0.25$. The inner face of the shell is driven by a pressure constraint of magnitude $10^{6} \mathrm{~Pa}$ whereas the outer face is a stress free boundary condition. The final time is $t_{\text {final }}=1.6 \cdot 10^{-4} \mathrm{~s}$. In practice, for computational time reasons, the domain is not a complete shell but a needle-like domain of one degree aperture angle. All the boundary faces introduced by this geometrical simplification are then symmetry boundary conditions. As such the computational domain is defined by $\omega=[r, \theta, \phi]=[0.9, \pi / 180, \pi / 180]$ and three meshes with characteristics length $h=1 / N_{s}$ are considered $\left(N_{c} \equiv N_{s}=1000 s\right.$ cells with $s=1,2,3)$. An additional difficulty arises in the context of three-dimensional unstructured meshes, which is related to the spatial discretization of the needle-like computational domain for the Blake problem. In order to avoid ill-conditioned reconstruction matrices due to the high difference in cell size between elements close to the origin of the needle and the ones very far from that location, the entire computational domain has to be mapped onto a reference system $[\bar{r}, \bar{\theta}, \bar{\psi}]$ such that all coordinates are defined within the interval $[0 ; 1]$. This is sufficient to carry out a second order reconstruction on a more uniform tessellation of the domain with tetrahedra. In figure 10 we present the mesh of the needle and the pressure distribution at final time as illustration with $N_{s}=1000$. In order to provide more quantitative analysis, in figure 11 we display the numerical results for the pressure and radial deviatoric stress (and zooms) as a function of radius for a sequence of meshes: $N_{1}=1000, N_{2}=2000$ and $N_{3}=3000$. The solution is then compared against the reference solution. We can observe not only accuracy but also convergence even though some perturbations are present for small radius on pressure variables. 

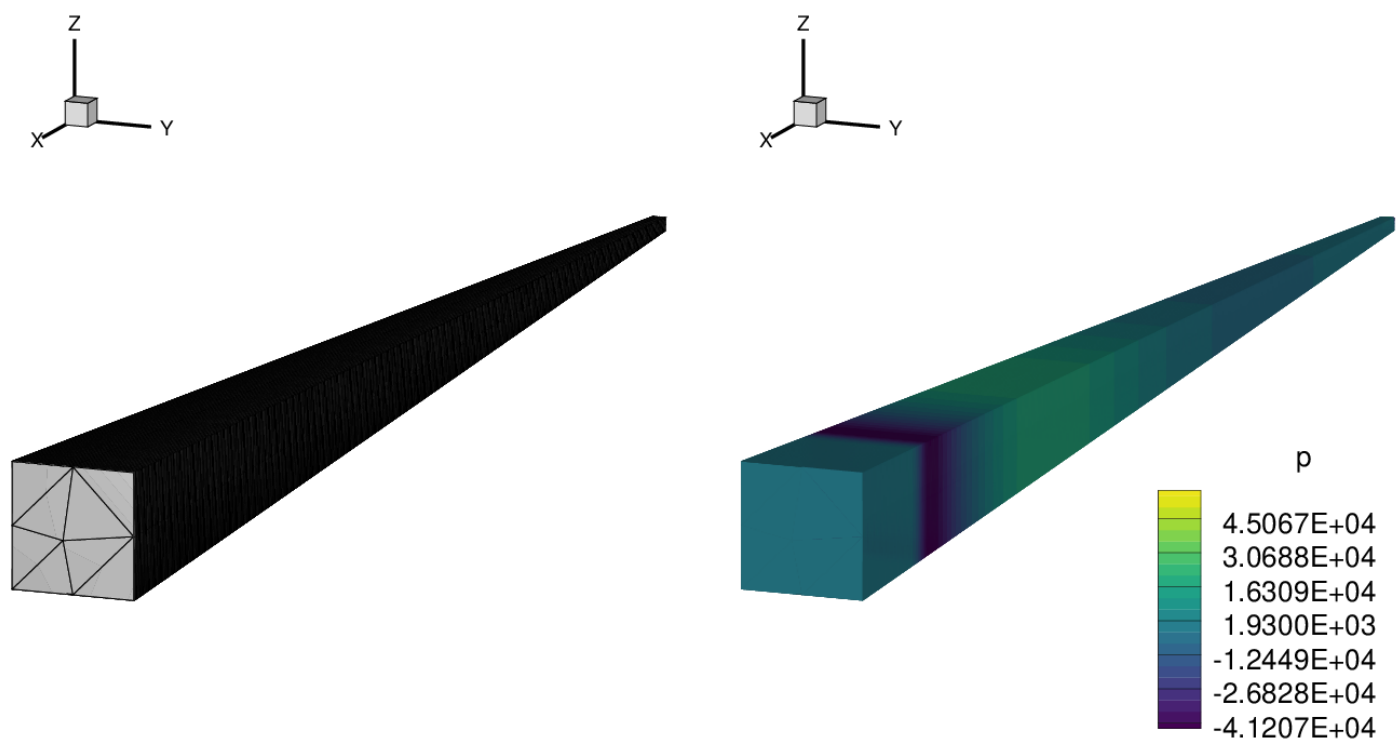

Fig. 10. Blake's problem - Computational mesh of the needle domain $\omega=[r, \theta, \phi]=[0.9, \pi / 180, \pi / 180]$ with $h=1 / 1000$ (left) and pressure distribution at the final time $t_{\text {final }}=1.6 \cdot 10^{-4} \mathrm{~s}$ (right).

\subsection{Twisting column}

A twisting column test case aims at examining the effectiveness of the proposed methodology in highly nonlinear scenarios, see [36] and the reference therein. An initial unit squared cross section column of height $H=6 \mathrm{~m}$ is considered, $\omega=[-0.5 ; 0.5] \times[-0.5 ; 0.5] \times[0 ; 6]$. The $z=0$ face of the column is embedded into a wall. An initial sinusoidal angular velocity field relative to the origin is given by $\boldsymbol{v}_{0}=100 \sin \left(\pi \frac{z}{2 H}\right)(y,-x, 0)^{t} \mathrm{rad} / \mathrm{s}$, see figure 12 . The main objective of this problem is to assess the capability of the proposed methodology to still perform when approaching the limit of incompressibility. A neo-Hookean material is used with material density $\rho_{0}=1100 \mathrm{~kg} \cdot \mathrm{m}^{-3}$, Young's modulus $E=1.7 \cdot 10^{7} \mathrm{~Pa}$ and Poisson's ratio $\nu=0.45$. The simulation is run till time $t_{\text {final }}=0.3 \mathrm{~s}$. Qualitatively one should observe at time $t \sim 0.1 \mathrm{~s}$ a counter-clockwise rotation and a severe twist of the column which returns to its initial position at about $t \sim 0.2 \mathrm{~s}$. Driven by its own inertia, the bar twists clockwise until the final time. The mesh of the column is made of $N_{c}=119092$ tetrahedra with characteristic length of $1 / 80$. Stress free BCs are imposed everywhere apart from the bottom face for which we impose a wall type boundary with zero displacement. In figure 13 we plot the shape of the column colored by the pressure distribution for different output times. The initial column is represented as a hollow bar for comparison purposes. The main behaviors are reproduced by the numerical simulation. Notice that there is no spurious oscillations nor suspicious pressure distribution. In figure 14 we gather several diagnostics of this simulation. First on the left panel we plot the time evolution of dimensionless height of the column measured at the point initially located at $\boldsymbol{x}_{T}=(0,0,6)$. Next, in the middle panel, we plot the numerical dissipation of the second-order scheme computed as the percentage of energy loss computed by means of (88) as a function of time and observe that at final time only $0.5 \%$ is lost. For a numerical simulation recall that the twisting period does not only depend on the material but also on the numerical dissipation of the scheme. Usually first-order schemes 

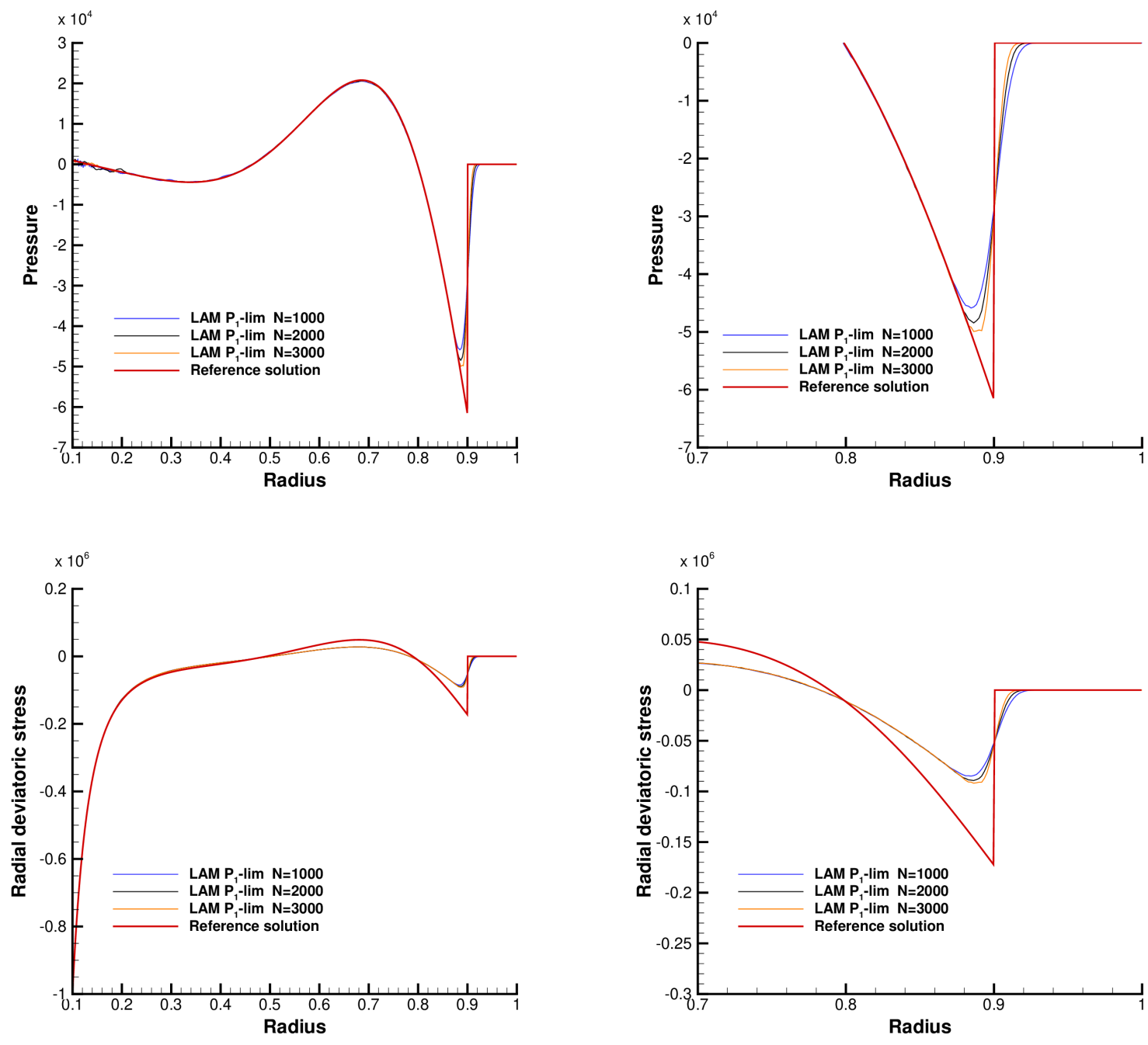

Fig. 11. Blake's problem - Mesh convergence of the second order solution towards the reference solution (red) for the radial pressure (top row) and radial deviatoric stress (bottom row) at time $t_{\text {final }}=1.6 \cdot 10^{-4}$ (left) and zoom across the shock (right). Remind that LAM $\mathbb{P}_{1}$-lim corresponds to cascade: $\mathbb{P}_{1} \rightarrow \mathbb{P}_{1}^{\lim } \rightarrow \mathbb{P}_{0}$.

are extremely dissipative and can not perform adequately, i.e. the column barely twists. At last in the right panel we present the percentage of bad cells detected at each time step by the a posteriori limiting procedure and observe that on average only $2 \%$ of the cells are recomputed due to spurious numerical issues.

\subsection{Rebound of a hollow circular bar}

Taken from [36] as the 3D extension of a 2D contact problem found in [24], the impacting bar test consists in the rebound of a hollow circular bar of outer diameter $6.4 \mathrm{~mm}$, inner diameter $2 \mathrm{~mm}$ and height $H=32.4 \mathrm{~mm}$, see figure 12. The bar impacts against a rigid friction-less wall with an initial velocity of $\boldsymbol{v}_{0}=(0,0,-100)^{t} \mathrm{~m} / \mathrm{s}$ and the separation distance between the bar and wall is $4 \mathrm{~mm}$. Before the impact time at $t=40 \mu \mathrm{s}$ the bar is on a ballistic flight. Upon impact, the bar undergoes large compressive deformation until $t=150 \mu$ s when all the kinetic energy of the bar is converted into internal strain energy. Afterwards, tensile forces develop and a bounce-off motion initiates in such a way that, at approximately $t \simeq 250 \mu \mathrm{s}$, 

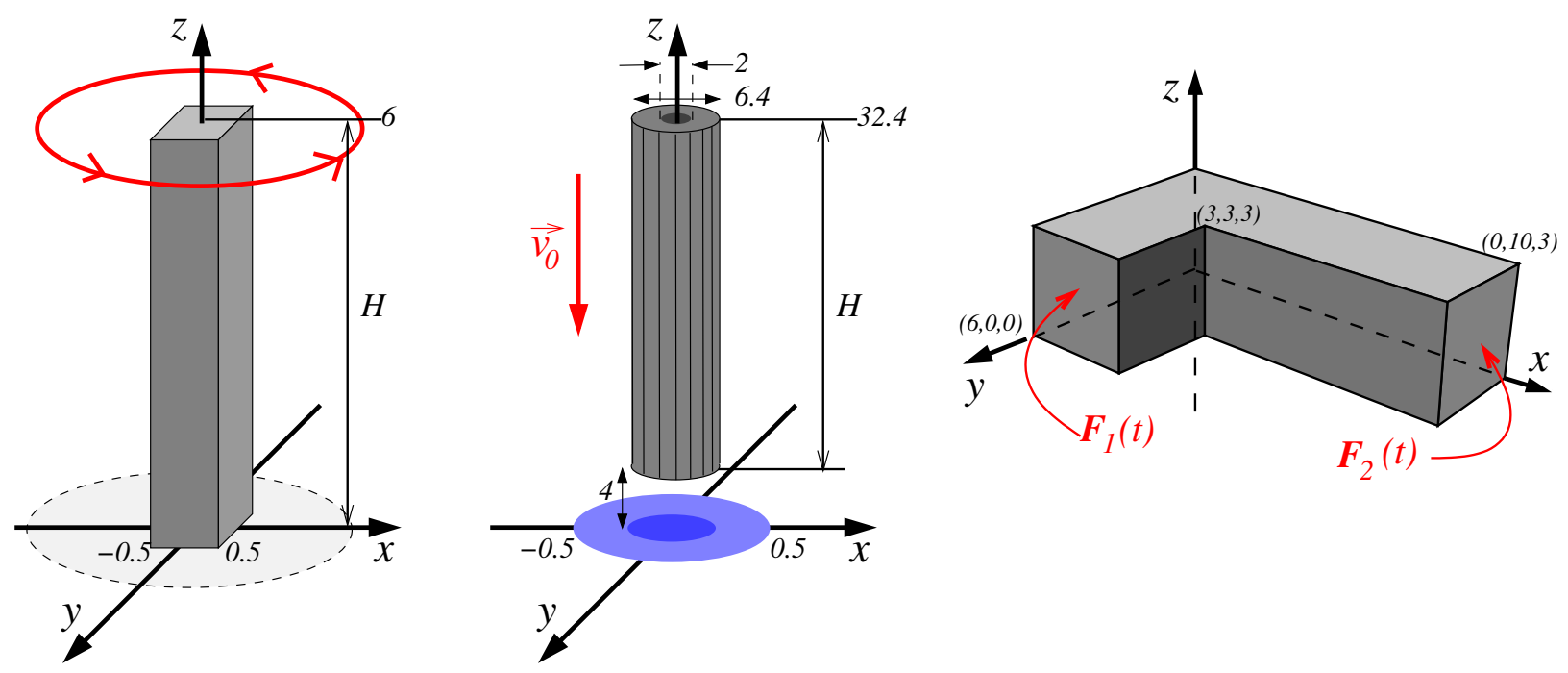

Fig. 12. Sketch for the twisting column in section 5.5 (left) and the rebound of a hollow circular bar from section 5.6 (middle), and, L-shaped block test problem from section 5.8 (right).

the bar completely detaches from the wall and moves upwards, still enduring internal milder deformations. The neo-Hookean constitutive model is chosen with density $\rho^{0}=8930 \mathrm{~kg} \cdot \mathrm{m}^{-3}$, Young's modulus $E=585 \mathrm{MPa}$ and Poisson's ratio $\nu=0.45$ and the final time is set to $325 \mu \mathrm{s}$.

The fixed wall is the $x-y$ plane and is considered as a restricted tangential displacement type BCs. The rest of the material is subject to free-traction BCs. Special care must be paid to the points of the inner circle at the bottom of the bar. Indeed for these points the BCs must evolve from free-traction to slip-wall BCs during the contact time up to detachment. Specifically, free-traction BCs are used until the velocity of the nodes lying on the bottom face is downward pointing and the distance to the wall is greater than a prescribed tolerance of $10^{-12}$. As soon as the new node position would exceed the $z$-coordinate of the wall, i.e. $z=0$, the time step is modified in order to let the bar exactly hit the wall, then the boundary condition switches to slip wall type from the next time step on. Then, when the velocity of the bottom face nodes becomes upward pointing because of the rebound of the bar, as soon as the new node position would detach from the wall, the time step is again modified so that it exactly matches the time of detachment and finally the boundary condition changes again to free-traction for the rest of the simulation. One quarter of the hollow bar is meshed with $N_{c}=12254$ tetrahedra and a characteristics length of $1 / 128$. In figure 15 we present the time evolution of the deformation and pressure distribution (colors) at times $t=50 \mu \mathrm{s}$ then $75,100,125,150,200,300$ and the final time $t=325 \mu \mathrm{s}$. The main behaviors and deformations are captured by the numerical simulations as compared to the results in [36]. Following [36] (see Fig. 27), we present on the left panel of figure 16 the time evolution of vertical displacement of the points on the top $\mathbf{x}_{T}=(1.6,0,32.4) \cdot 10^{-3} \mathrm{~m}$ (black) and bottom $\mathbf{x}_{B}=(1.6,0,4) \cdot 10^{-3} \mathrm{~m}$ (red) planes. The general behavior is again qualitatively reproduced. At last, on the right panel of figure 16, we show the percentage of bad cells detected by the a posteriori limiter and observe that, on average, less than $3 \%$ demands limiting at each iteration. This induces a rather efficient limiting procedure compared to classical a priori slope limiters. 

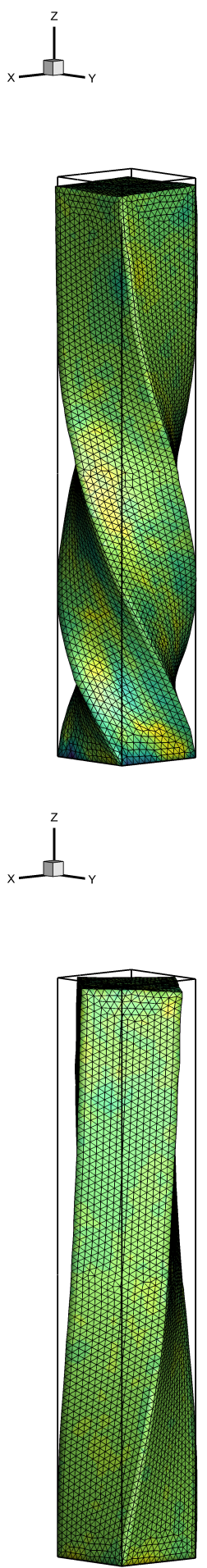
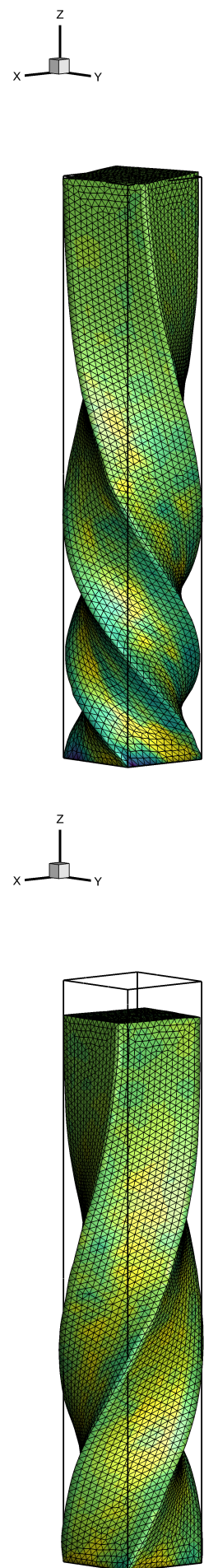
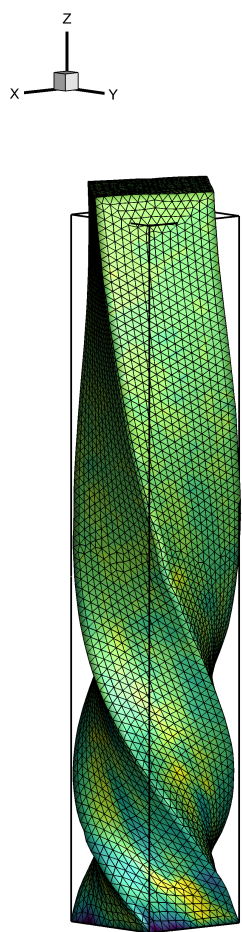

$\mathbb{1}_{x}^{2}$
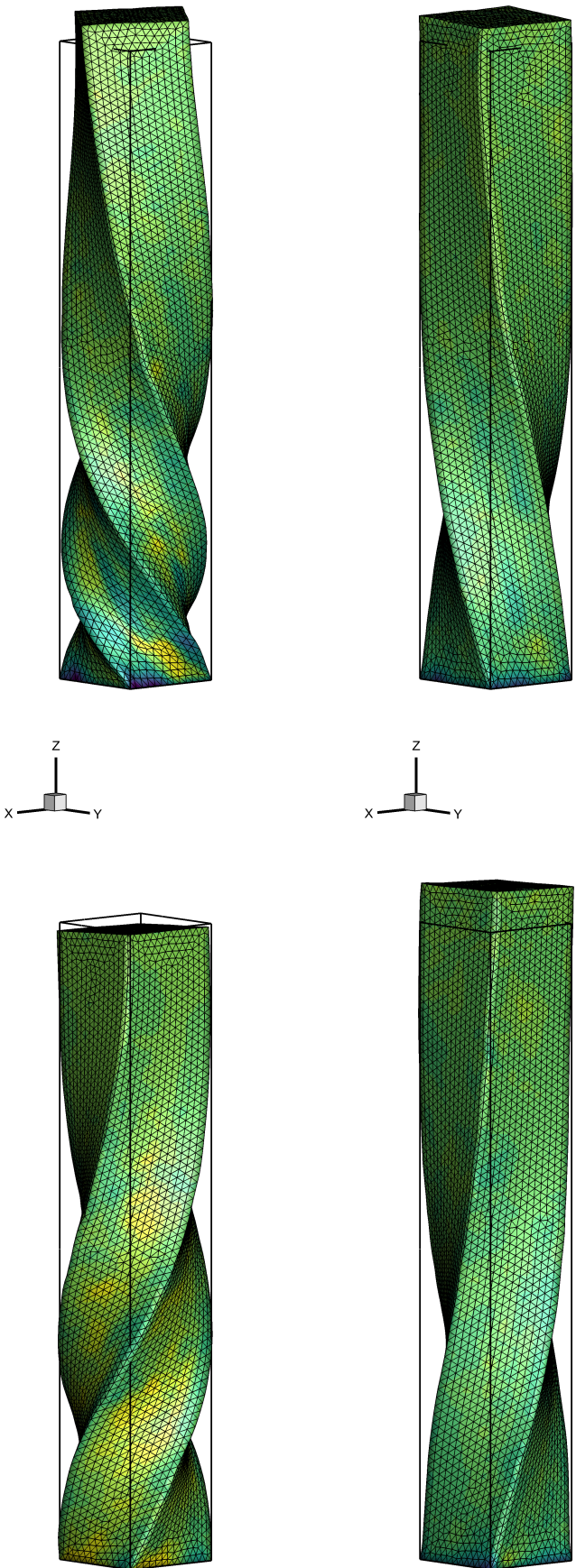

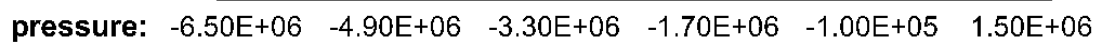

Fig. 13. Twisting column - Beam shape and pressure distribution at output times $t=0.00375$, $t=0.075, t=0.1125, t=0.15, t=0.1875, t=0.225, t=0.2625$ and $t=0.3$ (from top left to bottom right). The shape is compared with respect to the initial configuration (hollow box). 

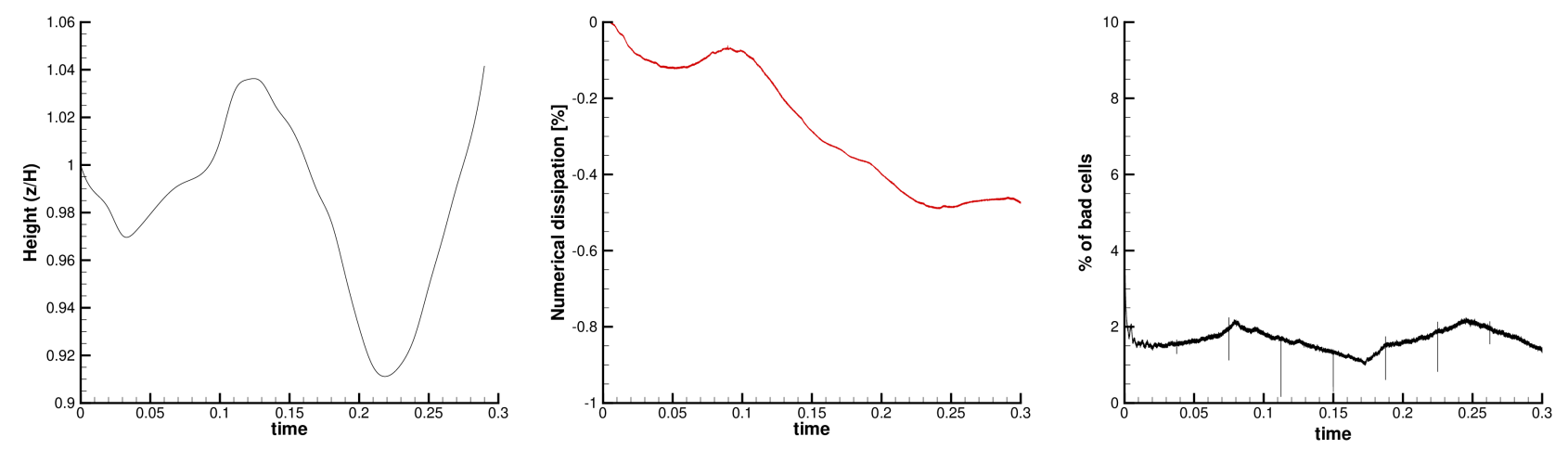

Fig. 14. Twisting column - Time evolution of non-dimensionalised height of the column measured at initial point $\mathbf{x}_{T}=(0,0,6)$ (left) - Numerical dissipation (88) of second order scheme (center) - Percentage of bad cells detected at each time step (right).

\subsection{Impact of a jelly-like droplet}

For this test case we consider the impact of a jelly-like material onto a flat rigid horizontal surface, inspired by the test in [51]. A cylinder of clay (bentonite) of diameter $L_{0}$ and height $h$ moves downward with velocity $\boldsymbol{v}=(0,-v) \mathrm{m} . \mathrm{s}^{-1}$, and material parameters $\gamma=2.2$, $p_{\infty}=10^{6}, \mu=85 \mathrm{~Pa}, \rho_{0}=1020 \mathrm{~kg} \cdot \mathrm{m}^{-3}$. Experiments of such impacts have been carried on in particular in [41] on different types of surface (smooth glass, hydrophobic). In such situation we are interested in the final diameter of the impacting droplet $L$ and the experimental results show a quasi-linear behavior of the maximal spread factor with respect to the impact velocity. Initially $L_{0}=12 \mathrm{~mm}$ and $h=8 \mathrm{~mm}$, and two impact velocities are considered, $v=2 \mathrm{~m} . \mathrm{s}^{-1}$ and $3 \mathrm{~m} \cdot \mathrm{s}^{-1}$. The numerical simulation considers a 3D computational domain constituted by an approximation of $1 / 4$ of the initial bentonite cylinder $\omega^{0}$ and a mesh made of $N_{c}=717396$ tetrahedra with characteristics length $1 / 100$. Two constitutive laws are tested, namely the neo-Hookean model, $a=-1$, and the non-linear one $a=0$, see section 2.2.3 for details. Symmetry BCs are imposed for the $x=0$ and $y=0$ planes, while free-traction BCs are applied on the top and cylinder boundaries and slip wall type is prescribed on the bottom side. Figure 17 depicts the shapes of the material for successive times $t=2 k \times 10^{-3} \mathrm{~s}$ for $0 \leq k \leq 5$ in the case of a $v=3 \mathrm{~m} \cdot \mathrm{s}^{-1}$ impact velocity. The black shape corresponds to the non-linear model $a=0$, while the petroleum shape corresponds to a neo-Hookean one $a=-1$. They are put in respect to each other for comparison purposes.

Regardless of the constitutive model, i.e. the value of $a$, the jelly-like material is compressed after the impact and deforms back and forth due to its elastic behavior. As expected with the neo-Hookean model (petroleum shape) the spread of the droplet is much more pronounced and the droplet retrieves a cylinder-like shape slower compared to the non-linear model (black shape). In order to quantify this behavior we present in figure 18 the maximum spreading of the droplet, $L / L_{0}$, in the case $a=-1$ (black line) and $a=0$ (red line) for the two impact velocities. The neo-Hookean model produces faster and more pronounced elastic behaviors compared to the non-linear model which retrieves a ratio closer to one faster. The experimental results in [41] provide approximate values 2.25 and 2.75 , respectively, while our simulations produce 1.8 and 2.5 in accordance to the numerical results in [51]. 

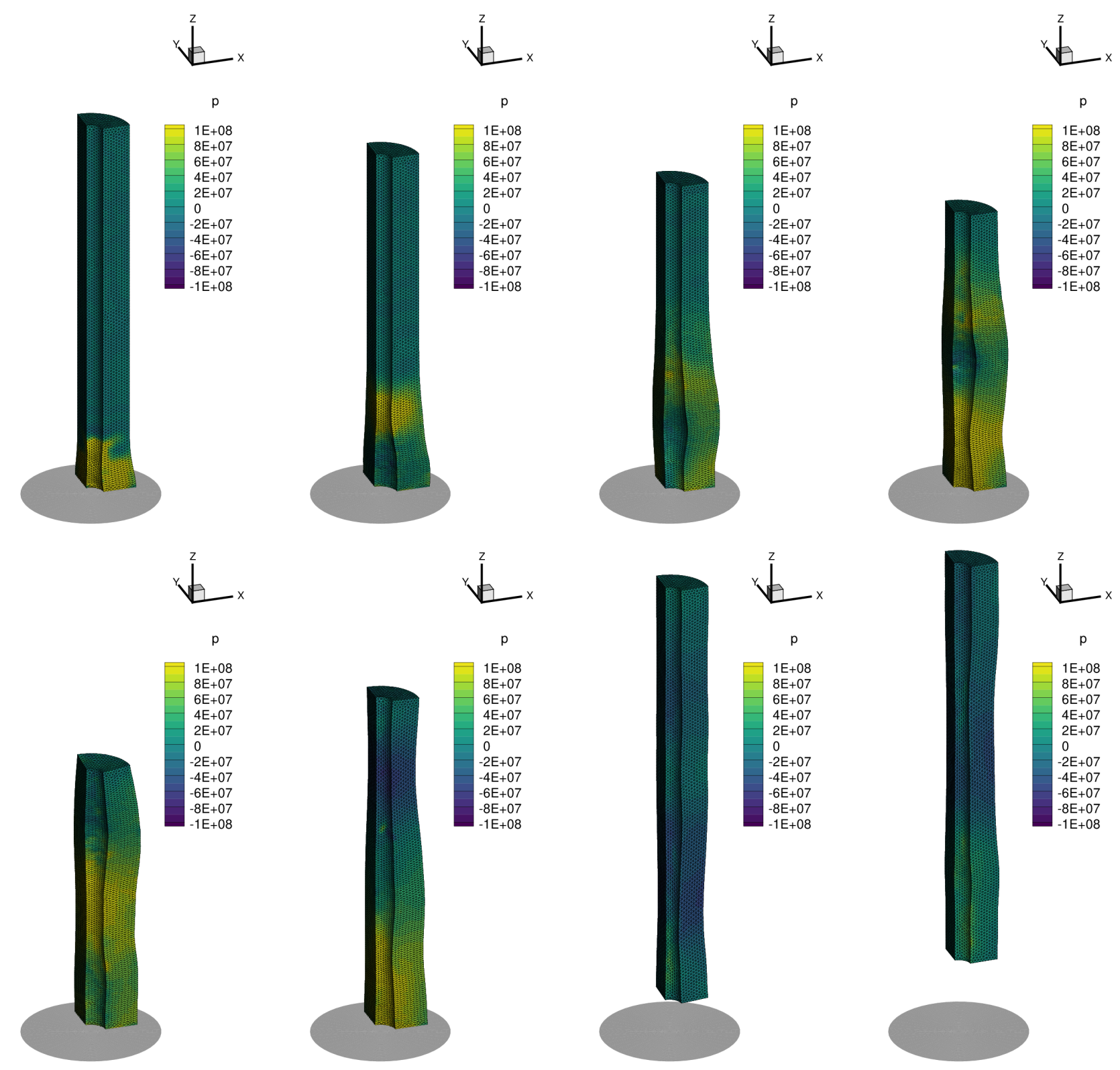

Fig. 15. Rebound of a hollow circular bar - Time evolution of the deformation and pressure distribution at output times at times $t=50 \mu$ s then 75, 100, 125, 150, 200, 300 and the final time $t=325 \mu \mathrm{s}$ (from top left to bottom right).

\subsection{L-shaped block}

To assess the linear and angular momentum preservation, a specific test case has been designed in $[8,33]$. It consists in simulating the motion of a 3D L-shaped block subjected to initial time-dependent impulse traction forces, $\boldsymbol{F}_{1}(t)$ and $\boldsymbol{F}_{2}(t)$, at two of its sides, creating de facto an external torque, see figure 12 . The traction forces are given by

$$
\boldsymbol{F}_{1}(t)=-\boldsymbol{F}_{2}(t)= \begin{cases}t \boldsymbol{e}, & 0 \leq t<2.5, \\ (5-t) \boldsymbol{e}, & 2.5 \leq t<5, \\ 0 \boldsymbol{e}, & t>5,\end{cases}
$$



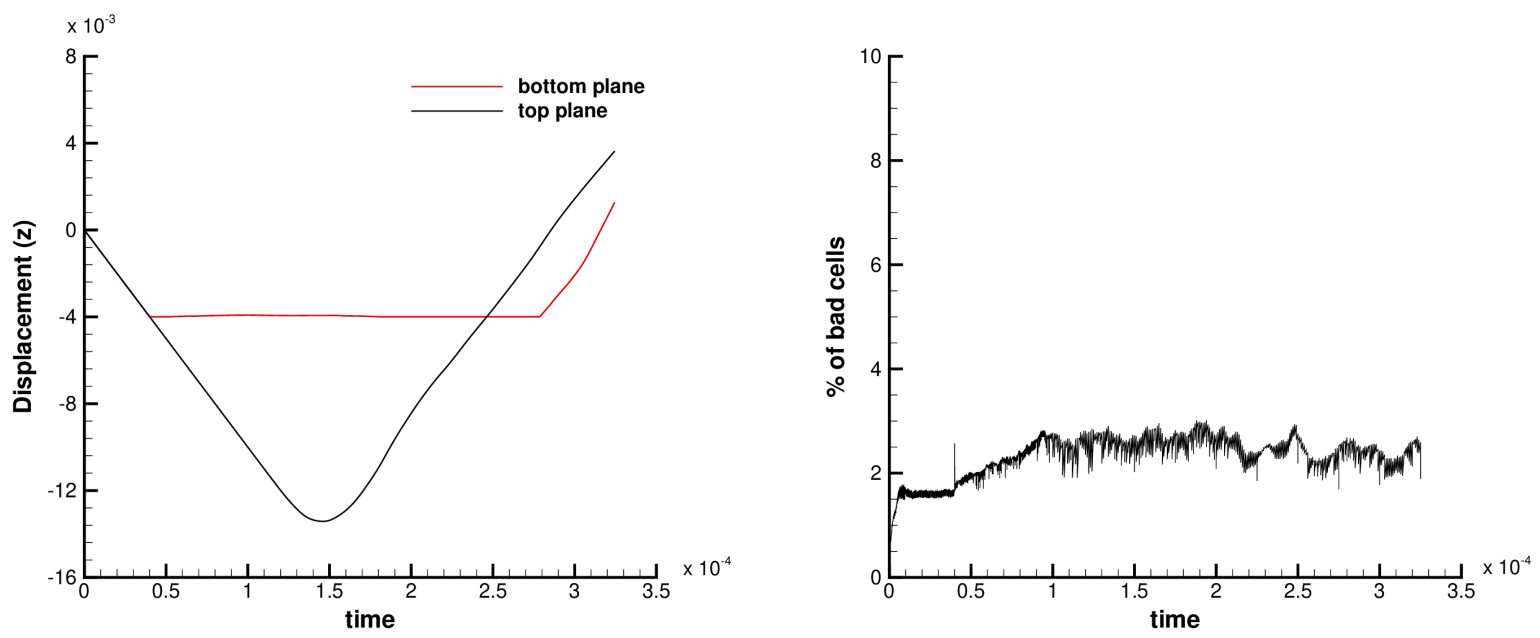

Fig. 16. Rebound of a hollow circular bar - Time evolution of vertical displacement of the points on the top plane $\mathbf{x}_{T}=(1.6,0,32.4) \cdot 10^{-3} \mathrm{~m}$ and on the bottom plane $\mathbf{x}_{B}=(1.6,0,4) \cdot 10^{-3} \mathrm{~m}$ (left) and percentage of bad cells detected at each time step (right).

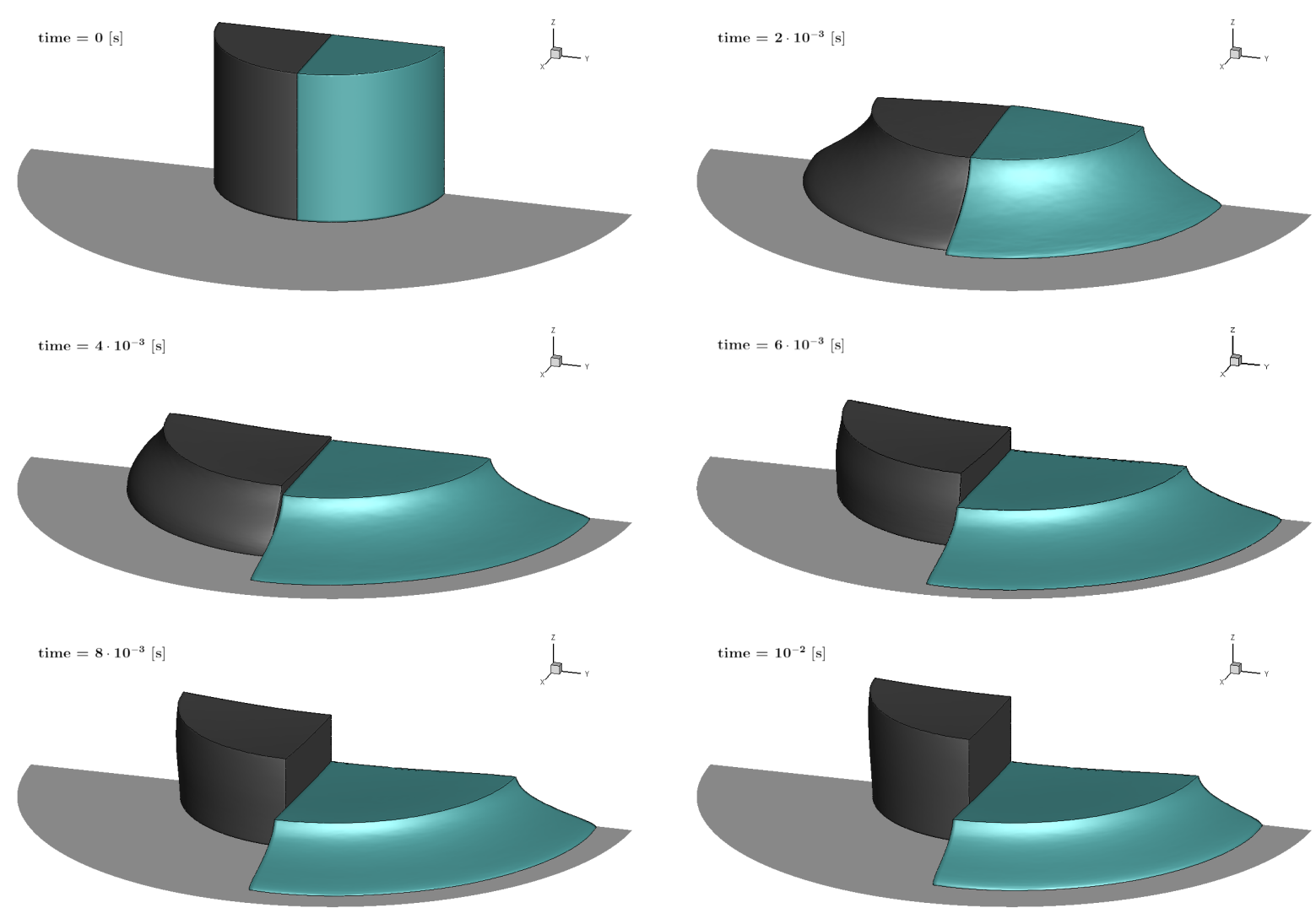

Fig. 17. Impact of a jelly droplet with impact velocity $3 \mathrm{~m} \cdot \mathrm{s}^{-1}$ - Time evolution of the droplet shape at different output times for neo-Hookean model $(a=-1$, petroleum shade) or non-linear one ( $a=0$, black shade).

where $\boldsymbol{e}=(150,300,450)^{t}$. The neo-Hookean material is governed by the following physical properties: $\rho^{0}=1000 \mathrm{~kg} . \mathrm{m}^{-3}$, Young's modulus $E=50046 \mathrm{~Pa}$ and Poisson ratio $\nu=0.3$. The final time is set to $70 \mathrm{~s}$, hence involving a quite long simulation time.

We consider a mesh made of 103811 tetrahedra with characteristic mesh size $h=1 / 50$. In figure 19 we present the numerical pressure and the shape of the block obtained at different 

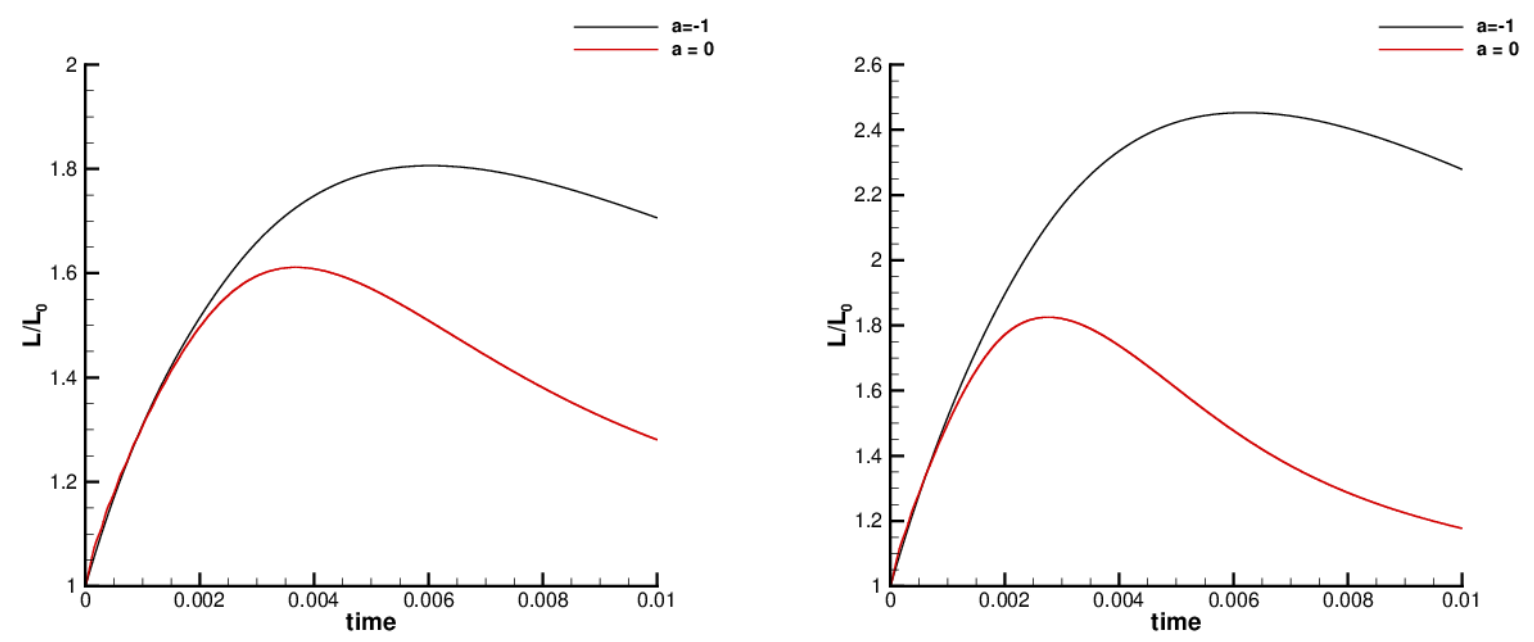

Fig. 18. Impact of a jelly droplet - Time evolution of the maximum spreading of the droplet $L / L_{0}$ in the case neo-Hookean model ( $a=-1$, black line) or non-linear one ( $a=0$, red line) — The impact velocity is $2 \mathrm{~m} \cdot \mathrm{s}^{-1}$ (left) and $3 \mathrm{~m} \cdot \mathrm{s}^{-1}$ (right).

discrete times $t=2.5,5.0,7.5,10.0,12.5$ and $15 \mathrm{~s}$. The results are in agreements with the ones presented for instance in [8,33]. Next in figure 19 we monitor the cell-centered angular momentum $\boldsymbol{A}$ with respect of $x, y$ and $z$ axes, computed as

$$
\boldsymbol{A}=\left(A_{x}, A_{y}, A_{z}\right)=\int_{\omega} \boldsymbol{x} \times m \boldsymbol{v} \mathrm{d} v \approx \sum_{c} \boldsymbol{x}_{c} \times m_{c} \boldsymbol{v}_{c}\left|\omega_{c}\right|=\boldsymbol{A}_{c}
$$

In the figure we clearly see that after $t=5$, when the boundary forces vanish, the angular momenta are nicely preserved even if nothing in the method has been designed to ensure so. Those results are in agreement with the ones from $[8,33]$ for instance. Notice that the linear momentum is preserved up to machine precision and we omit these results.

\subsection{Monitoring of the internal consistency}

At last, as explained in section 3.6, we monitor the internal consistency of the scheme by the computation of $\epsilon_{\mathbb{B}}$ from (85) for the 2D and 3D tests presented in this work. In figure 21 the 2D tests (left panel) and the 3D ones (right panel) are gathered for the first 75000 time-steps. In general, in $2 \mathrm{D}$ or $3 \mathrm{D}$, the value of $\epsilon_{\mathbb{B}}$ remains under a small threshold (always less than $10^{-11}$ ), see table 3 for the maximal values. Moreover for the two $2 \mathrm{D}$ problems we compare our results to the ones given by a genuine first order Lagrangian scheme. As expected the value of $\epsilon_{\mathbb{B}}$ is larger by 7 orders of magnitude, justifying the use of a higher order scheme to maintain the error in internal consistency of the simulation at a negligible level. Table 3 reports the maximum error in time through the entire simulations for both 2D and 3D tests, demonstrating that it remains at machine precision and thus that the internal consistency of the update of $\mathbb{B}$ is satisfied by our second order scheme. 

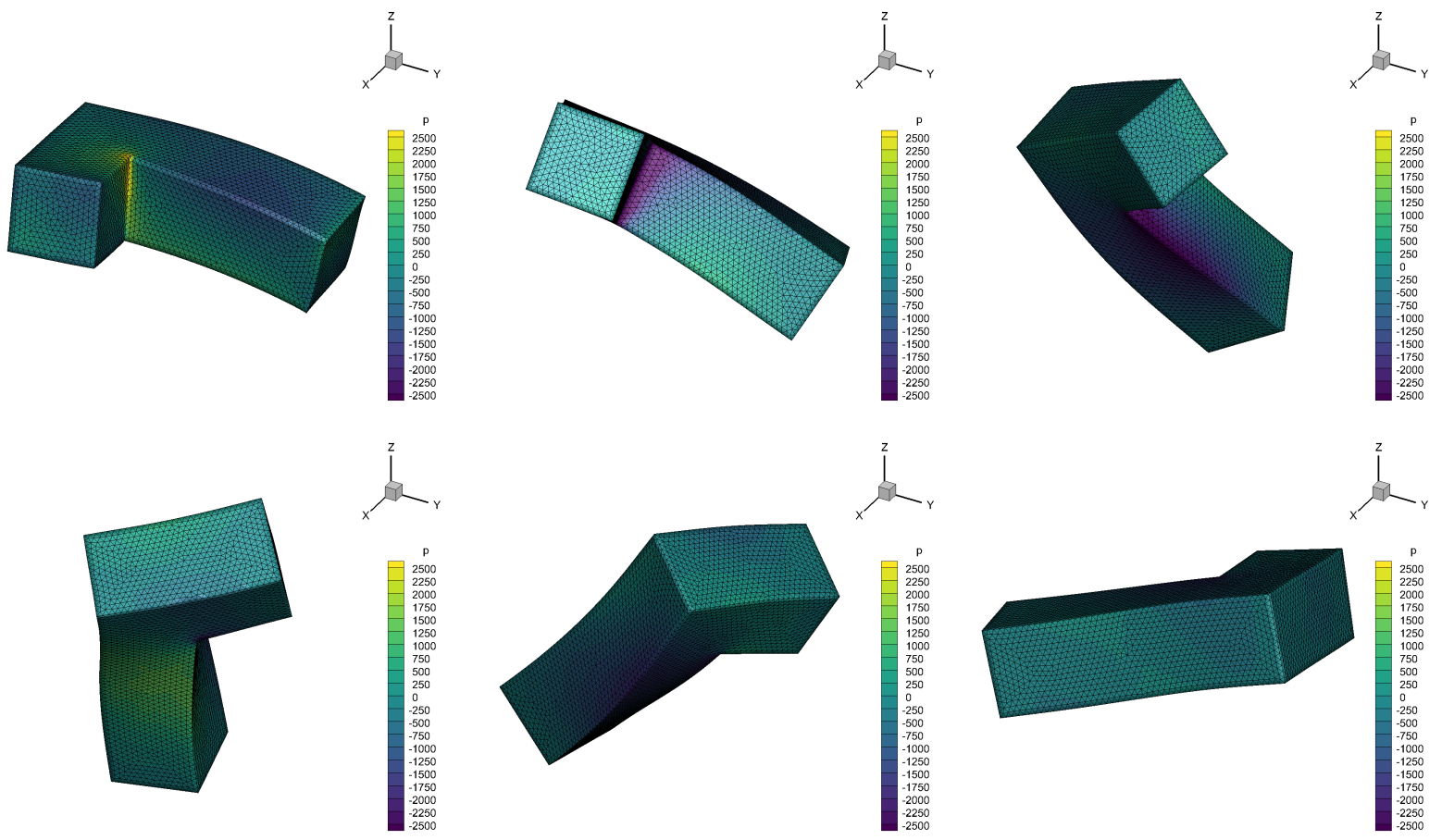

Fig. 19. L-shaped block test problem - From top-left to bottom-right: time $t=2.5,5.0,7.5,10.0$, 12.5 and $15 \mathrm{~s}$.

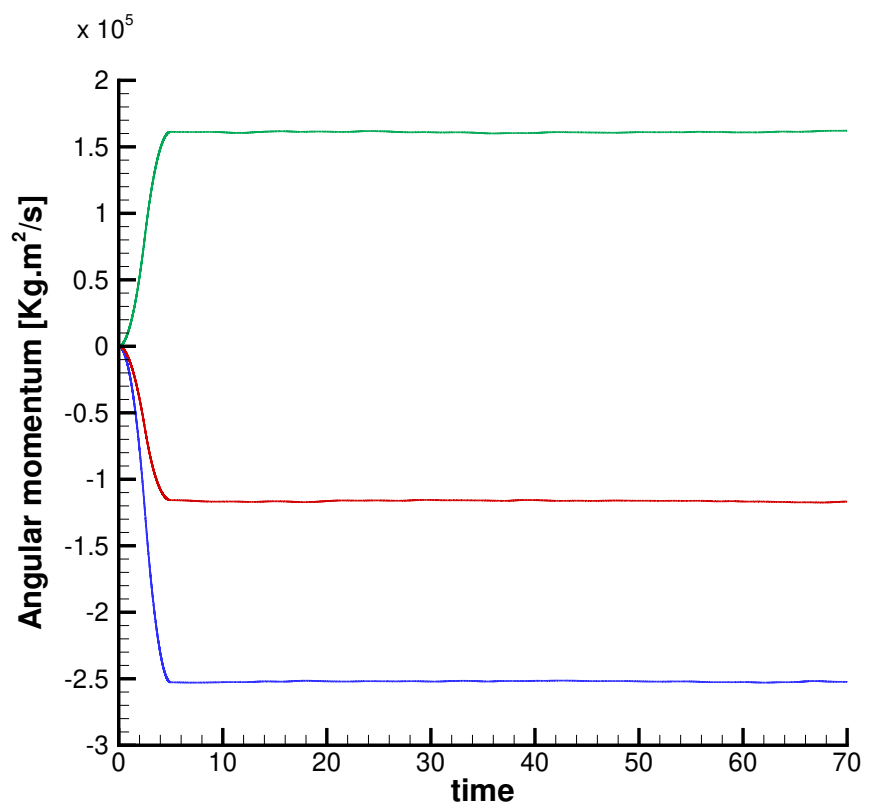

Fig. 20. L-shaped block test problem - Angular momentum $\boldsymbol{A}_{c}$ in $x$ (green), $y$ (red) and $z$ (blue) directions as a function of time.

\begin{tabular}{|c|cccccc|}
\hline Test case & Beryllium plate & Thick beam & Bar rebound & Blake & Jelly impact & Twisting column \\
\hline $\max \left(\epsilon_{\mathbb{B}}\right)$ & $5.86 \times 10^{-13}$ & $3.08 \times 10^{-13}$ & $1.12 \times 10^{-12}$ & $9.85 \times 10^{-12}$ & $4.19 \times 10^{-12}$ & $4.81 \times 10^{-13}$ \\
\hline
\end{tabular}

Table 3

Maximal value of $\epsilon_{\mathbb{B}}$ for the first 75000 time-steps of the 2D and 3D tests cases in this paper. 

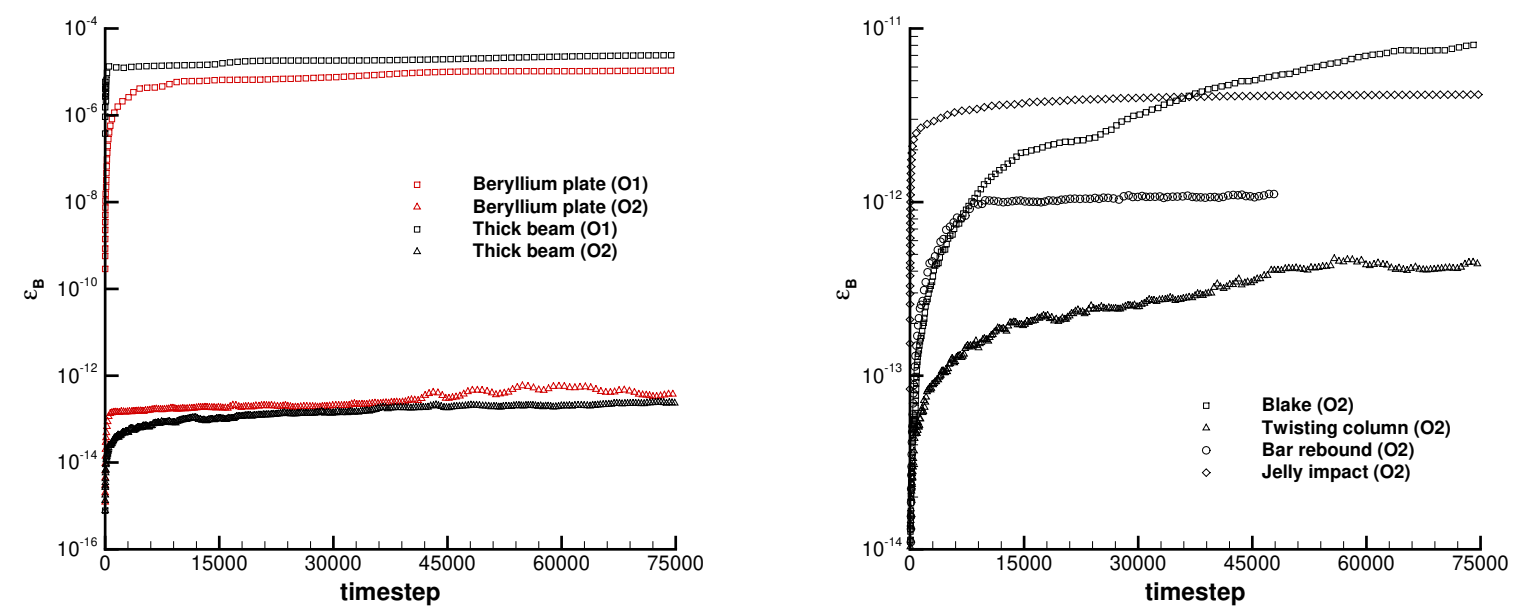

Fig. 21. Time evolution of the internal consistency condition (85): $\epsilon_{\mathbb{B}}=\left|\sqrt{\operatorname{det} \mathbb{B}_{c}^{n+1}}-\frac{\tau_{c}^{0}}{\tau_{c}^{n+1}}\right|$ for the 2D (left) and 3D (right) simulations presented in this article - Only the first 75000 time steps are shown - In 2D a comparison between a classical first order Lagrangian scheme and our current nominally second order one is shown.

\section{Conclusions and perspectives}

This paper considers the second-order accurate cell-centered Lagrangian scheme originally designed for the hydrodynamics system of conservation laws [13], and, extends it to solve the hyperelasticity model for materials in 2D and 3D. We have focused the first part of the paper on presenting the hyperelasticity model and its consistency in the Lagrangian frame. The so-called neo-Hookean model is mostly considered in this work. Then the numerical method based on a conservative Lagrangian formulation in mass, momentum and total energy is presented. It is supplemented with a nodal solver allowing the determination of a vertex velocity used to build a consistent discretization between the trajectory equation and the geometrical conservation law. Second-order of accuracy in space and time is achieved via an ADER procedure which generates a predictor solution that can further be used inside the classical subcell force based Lagrangian scheme with nodal solver. Robustness and stability are gained by the use of an a posteriori MOOD limiting strategy, that is a second-order unlimited candidate solution at $t^{n+1}$ is tested against appropriate detection criteria to determine troubled cells. The solution in those cells is discarded and re-computed starting again from valid data at $t^{n}$ but using a second-order TVD like scheme or, ultimately, the fail-safe first-order parachute scheme. The time evolution of the left-Cauchy Green tensor required for the isotropic hyperelastic constitutive law is discretized with great care to ensure its compatibility with the Geometric Conservation Law (GCL) at the discrete level. Moreover evolving boundary conditions have been implemented to allow for impacting and detaching of materials onto walls.

This numerical scheme has been further implemented in 2D and 3D under MPI protocol for the parallelization. It has been then tested on unstructured simplicial meshes on a large panel of 2D test cases: swinging plate, elastic vibration of a beryllium plate and a finite deformation of a cantilever thick beam. Then, in 3D, we have presented the results for Blake's 
problem, the twisting column, the rebound of a hollow circular bar, the impact of a jelly-like droplet and at last the L-shaped block test to assess the ability of our FV scheme to conserve angular momentum. This test suite covers a large amount of situations involving elastic materials and the current Lagrangian numerical scheme has proven to be robust, essentially non-oscillatory and, at the same time maintains an almost optimal precision by a careful utilization of the high order scheme where appropriate and the low order ones in the vicinity of problematic zones. Moreover its performance in 2D/3D both in terms of robustness, efficiency and compliance with other published results renders this numerical method appealing for future uses and possible coupling with more complex physical models.

A plan for future work involves the introduction of plasticity into this hyperelasticity model. We also plan to investigate the high-order extension over curvilinear simplicial grids of the present FV discretization. Another direction of evolution would be to add some ArbitraryLagrangian-Eulerian capability and the possibility to let two elastic materials interacting with each other, for instance impacting, deforming and further detaching from each others.

\section{Acknowledgments}

The material of this research has been partly built during the SHARK FV workshops in May 2017, 2018 and 2019 in Povoa de Varzim, Portugal www.SHARK-FV.eu/home-shark/. WB would like to thank the Italian Ministry of Instruction, University and Research (MIUR) to support this research with funds coming from PRIN Project 2017 No. 2017KKJP4X entitled "Innovative numerical methods for evolutionary partial differential equations and applications".

\section{A Principal invariants of a tensor}

Let us consider an invertible second order tensor $\mathbb{A}$. Its principal invariants are the coefficients of its characteristic polynomial

$$
\operatorname{det}\left(\mathbb{A}-\lambda \mathbb{I}_{\mathrm{d}}\right)=\lambda^{3}-I_{1}(\mathbb{A}) \lambda^{2}+I_{2}(\mathbb{A}) \lambda-I_{3}(\mathbb{A}) .
$$

They are defined by

$$
\begin{aligned}
& I_{1}(\mathbb{A})=\operatorname{tr}(\mathbb{A}), \\
& I_{2}(\mathbb{A})=\frac{1}{2}\left[\operatorname{tr}^{2}(\mathbb{A})-\operatorname{tr}\left(\mathbb{A}^{2}\right)\right], \\
& I_{3}(\mathbb{A})=\operatorname{det}(\mathbb{A}) .
\end{aligned}
$$

Moreover, the Cayley-Hamilton theorem states that $\mathbb{A}$ satisfies its characteristic equation

$$
\mathbb{A}^{3}-I_{1}(\mathbb{A}) \mathbb{A}^{2}+I_{2}(\mathbb{A}) \mathbb{A}-I_{3}(\mathbb{A}) \mathbb{I}_{\mathrm{d}}=0
$$


The derivative of the principal invariants of $\mathbb{A}$ with respect to itself write

$$
\begin{aligned}
& \frac{\partial I_{1}(\mathbb{A})}{\partial \mathbb{A}}=\mathbb{I}_{\mathrm{d}}, \\
& \frac{\partial I_{2}(\mathbb{A})}{\partial \mathbb{A}}=I_{1}(\mathbb{A}) \mathbb{I}_{\mathrm{d}}-\mathbb{A}, \\
& \frac{\partial I_{3}(\mathbb{A})}{\partial \mathbb{A}}=I_{3}(\mathbb{A}) \mathbb{A}^{-1} .
\end{aligned}
$$

\section{B Boundary conditions (BCs)}

In this cell-centered Lagrangian scheme, boundary conditions are imposed in the nodal solver $(57)$. Let $\mathcal{F}^{B C}(p)$ represent the set of boundary edges (or faces in 3D) surrounding node $p$. Three type of BCs are considered in this work.

- Traction: a prescribed traction $\mathbb{T}_{f}^{B C}$ on a boundary face $f$ is taken into account as an additional term on the right hand side as

$$
\boldsymbol{v}_{p}=\mathbb{M}_{p}^{-1}\left(\sum_{c \in \mathcal{C}(p)} \mathbb{M}_{p c} \boldsymbol{v}_{c}-\sum_{f \in \mathcal{F}(p) / \mathcal{F} B C} \mathbb{T}_{p c f} a_{p c f} \boldsymbol{n}_{p c f}\right)-\sum_{f \in \mathcal{F} B C(p)} \mathbb{T}_{p c f}^{B C} a_{p c f} \boldsymbol{n}_{p c f}
$$

- Velocity: the prescribed velocity $v_{p c f}^{B C}:=\boldsymbol{v}_{p c f}^{B C} \cdot \boldsymbol{n}_{p c f}^{n}$ can be interpreted as a traction BCs. The equivalent traction $\mathbb{T}_{\boldsymbol{v}}^{B C}$ is given by

$$
\mathbb{T}_{\boldsymbol{v}}^{B C}=\frac{\mathbb{M}_{p}^{-1}\left(\sum_{f \in \mathcal{F}(p)} \mathbb{T}_{p c f} a_{p c f} \boldsymbol{n}_{p c f}+\mathbb{M}_{p} \boldsymbol{u}_{p}\right) \cdot \boldsymbol{d}_{p}-\sum_{f \in \mathcal{F}^{B C}(p)} v_{p c f}^{B C} a_{p c f}}{\mathbb{M}_{p}^{-1} \boldsymbol{d}_{p} \cdot \boldsymbol{d}_{p}},
$$

where $\boldsymbol{d}_{p}=\sum_{f \in \mathcal{F}^{B C}(p)} a_{p c f} \boldsymbol{n}_{p c f}$ represents the corner vector associated to the boundary faces. Then, the node velocity is evaluated by considering

$$
\boldsymbol{v}_{p}=\mathbb{M}_{p}^{-1}\left(\sum_{c \in \mathcal{C}(p)} \mathbb{M}_{p c} \boldsymbol{v}_{c}-\sum_{f \in \mathcal{F}(p)} \mathbb{T}_{p c f} a_{p c f} \boldsymbol{n}_{p c f}\right)-\mathbb{T}_{\boldsymbol{v}}^{B C} \boldsymbol{d}_{p}
$$

- Symmetry: symmetry BC involves geometric considerations; either a symmetry plane defined by an orthonormal basis $\left(\boldsymbol{\tau}_{1}, \boldsymbol{\tau}_{2}\right)$, or a symmetry line along a direction vector $\boldsymbol{\tau}_{1}$, or even a symmetry point where we simply set $\boldsymbol{v}_{p}=\mathbf{0}$. In the case of a symmetry plane then the node velocity writes $\boldsymbol{v}_{p}=\alpha_{1} \boldsymbol{\tau}_{1}+\alpha_{2} \boldsymbol{\tau}_{2}$ and the momentum balance equation becomes

$$
\mathbb{M}_{p} \boldsymbol{v}_{p}=\mathbb{M}_{p}\left(\alpha_{1} \boldsymbol{\tau}_{1}+\alpha_{2} \boldsymbol{\tau}_{2}\right)=\left(\mathbb{M}_{p c f} \boldsymbol{v}_{c}-\sum_{f \in \mathcal{F}(p)} \mathbb{T}_{p c f} a_{p c f} \boldsymbol{n}_{p c f}\right)
$$

which is solved by successive projection on $\boldsymbol{\tau}_{1}$ and $\boldsymbol{\tau}_{2}$. On a symmetry line one has $\boldsymbol{u}_{p}=$ $\alpha_{1} \boldsymbol{\tau}_{1}$, that is $\alpha_{2}=0$.

For further details and comments on BCs we refer the reader to [46,31] and [13]. 


\section{References}

[1] M. Aguirre, A.J. Gil, J. Bonnet, and A. Arranz Carre no. A vertex-centred finite volume jameson-schmidt-turkel algorithm for a mixed conservation formulation in solid dynamics. $J$. Comput. Phys., 259:672-699, 2014.

[2] A.J. Barlow, P.-H. Maire, W.J. Rider, R.N. Rieben, and M.J. Shashkov. Arbitrary LagrangianEulerian methods for modeling high-speed compressible multimaterial flows. J. Comput. Phys., 322:603-665, 2016.

[3] T.J. Barth and D.C. Jespersen. The design and application of upwind schemes on unstructured meshes. AIAA Paper 89-0366, pages 1-12, 1989.

[4] A.L. Bauer, D.E. Burton, E.J. Caramana, R. Loubère, M.J. Shashkov, and P.P. Whalen. The internal consistency, stability, and accuracy of the discrete, compatible formulation of Lagrangian hydrodynamics. J. Comput. Phys., 218(2):572-593, 2006.

[5] T. Belytschko, L. Wing Kam, and B. Moran. Nonlinear Finite Elements for Continua and Structures. John Wiley \& Sons, Ltd, 2000.

[6] D. J. Benson. Computational methods in Lagrangian and Eulerian hydrocodes. Computer Methods in Applied Mechanics and Engineering, 99(2):235-394, 1992.

[7] Barry Bernstein. Hypo-elasticity and elasticity. Arch. Rational Mech. Anal., 6:89-104, 1960. https://doi.org/10.1007/BF00276156.

[8] J. Bonet, A. J. Gil, C. Hean Lee, M. Aguirre, and R. Ortigosa. A first order hyperbolic framework for large strain computational solid dynamics. part I: Total Lagrangian isothermal elasticity. Computer Methods in Applied Mechanics and Engineering, 283:689-732, January 2015 .

[9] J. Bonet, C. Hean Lee, A. J. Gil, and A. Ghavamian. A first order hyperbolic framework for large strain computational solid dynamics. part III: Thermo-elasticity. Computer Methods in Applied Mechanics and Engineering, 373:113505, January 2021.

[10] W. Boscheri and M. Dumbser. Arbitrary-Lagrangian-Eulerian One-Step WENO Finite Volume Schemes on Unstructured Triangular Meshes. Communications in Computational Physics, 14:1174-1206, 2013.

[11] W. Boscheri and M. Dumbser. A direct Arbitrary-Lagrangian-Eulerian ADER-WENO finite volume scheme on unstructured tetrahedral meshes for conservative and non-conservative hyperbolic systems in 3D. J. Comput. Phys., 275:484-523, 2014.

[12] W. Boscheri, M. Dumbser, and R. Loubère. Cell centered direct Arbitrary-Lagrangian-Eulerian ADER-WENO finite volume schemes for nonlinear hyperelasticity. Computers and Fluids, 134135:111-129, 2016.

[13] W. Boscheri, M. Dumbser, R. Loubère, and P.-H. Maire. A second-order cell-centered Lagrangian ADER-MOOD finite volume scheme on multidimensional unstructured meshes for hydrodynamics. J. Comput. Phys., 358:103 - 129, 2018.

[14] J. Breil, G. Georges, and P.-H. Maire. 3D cell-centered Lagrangian second order scheme for the numerical modeling of hyperelasticity system. Computer and Fluids, 207, 2020. 
[15] D.E. Burton. Exact conservation of energy and momentum in staggered-grid hydrodynamics with arbitrary connectivity. Advances in the Free Lagrange Method, Springer-Verlag, New-York, 1990.

[16] E.J. Caramana, D.E. Burton, M.J. Shashkov, and P.P. Whalen. The construction of compatible hydrodynamics algorithms utilizing conservation of total energy. Journal of Computational Physics, 146:227-262, 1998.

[17] E.J. Caramana, C.L. Rousculp, and D.E. Burton. A compatible, energy and symmetry preserving Lagrangian hydrodynamics algorithm in three-dimensional Cartesian geometry. Journal of Computational Physics, 157:89 - 119, 2000.

[18] G. Carré, S. Del Pino, B. Després, and E. Labourasse. A cell-centered Lagrangian hydrodynamics scheme on general unstructured meshes in arbitrary dimension. Journal of Computational Physics, 228:5160-5183, 2009.

[19] I. Castañar, J. Baiges, and R. Codina. A stabilized mixed finite element approximation for incompressible finite strain solid dynamics using a total Lagrangian formulation. Computer Methods in Applied Mechanics and Engineering, 368:113164, 2020.

[20] Jun-Bo Cheng, Yueling Jia, Song Jiang, Eleuterio F. Toro, and Ming Yu. A second-order cell-centered Lagrangian method for two-dimensional elastic-plastic flows. Communications in Computational Physics, 22(5):12241257, 2017.

[21] S. Clain, S. Diot, and R. Loubère. A high-order finite volume method for systems of conservation laws with multi-dimensional optimal order detection (MOOD). J. Comput. Phys., 230(10):4028 $-4050,2011$.

[22] B. Després. Numerical Methods for Eulerian and Lagrangian Conservation Laws. Frontiers in Mathematics. Springer International Publishing, 2017.

[23] B. Després and C. Mazeran. Lagrangian gas dynamics in two dimensions and Lagrangian systems. Arch. Rational Mech. Anal., 178:327-372, 2005.

[24] J. Donea and A.Huerta. Finite Element Methods for Flow Problems. Wiley, April 2003.

[25] M. Dumbser, M. Castro, C. Parés, and E.F. Toro. ADER schemes on unstructured meshes for non-conservative hyperbolic systems: Applications to geophysical flows. Computers and Fluids, $38: 1731-1748,2009$.

[26] M. Dumbser and M. Käser. Arbitrary high order non-oscillatory finite volume schemes on unstructured meshes for linear hyperbolic systems. Journal of Computational Physics, 221:693$723,2007$.

[27] D. P. Flanagan and T. Belytschko. A uniform strain hexahedron and quadrilateral with orthogonal hourglass control. International Journal for Numerical Methods in Engineering, 17(5):679-706, 1981.

[28] F.Vilar, P.-H. Maire, and R. Abgrall. A discontinuous Galerkin discretization for solving the two-dimensional gas dynamics equations written under total Lagrangian formulation on general unstructured grids. Journal of Computational Physics, 276:188-234, 2014.

[29] S. Gavrilyuk, S. Ndanou, and S. Hank. An example of a one-parameter family of rank-one convex stored energies for isotropic compressible solids. Journal of Elasticity, 124(1):133-141, December 2015. 
[30] S.L. Gavrilyuk, N. Favrie, and R. Saurel. Modelling wave dynamics of compressible elastic materials. Journal of Computational Physics, 227:2941-2969, 2008.

[31] G. Georges, J. Breil, and P.-H. Maire. A 3D GCL compatible cell-centered Lagrangian scheme for solving gas dynamics equations. Journal of Computational Physics, 305:921-941, 2016.

[32] C. Geuzaine and J.-F. Remacle. Gmsh: A 3-D finite element mesh generator with built-in preand post-processing facilities. International Journal for Numerical Methods in Engineering, 79(11):1309-1331, 2009.

[33] A. J. Gil, C. Hean Lee, J. Bonet, and R. Ortigosa. A first order hyperbolic framework for large strain computational solid dynamics. part II: Total Lagrangian compressible, nearly incompressible and truly incompressible elasticity. Computer Methods in Applied Mechanics and Engineering, 300:146-181, March 2016.

[34] G.L. Goudreau and J.O. Hallquist. Recent developments in large-scale finite element Lagrangian hydrocode technology. Computer Methods in Applied Mechanics and Engineering, 33(1):725-757, 1982.

[35] M.E. Gurtin, E. Fried, and L. Anand. The Mechanics and Thermodynamics of Continua. Cambridge University Press, 2010.

[36] J. Haider, C. Hean Lee, A. J. Gil, A. Huerta, and J. Bonet. An upwind cell centred total Lagrangian finite volume algorithm for nearly incompressible explicit fast solid dynamic applications. Computer Methods in Applied Mechanics and Engineering, 340:684 - 727, 2018.

[37] J.R. Kamm and L. Ankeny. Analysis of the Blake problem with RAGE. Technical Report LA-UR-09-01255, Los Alamos National Laboratory, 2009.

[38] G. Karypis and V. Kumar. Multilevel k-way partitioning scheme for irregular graphs. J. Parallel Distrib. Comput., 48:96-129, 1998.

[39] G. Kluth and B. Després. Discretization of hyperelasticity on unstructured mesh with a cellcentered Lagrangian scheme. Journal of Computational Physics, 229(24):9092 - 9118, 2010.

[40] C.H. Lee, A.J. Gil, and J. Bonet. Development of a stabilised Petrov-Galerkin formulation for conservation laws in Lagrangian fast solid dynamics. Comput. Methods Appl. Mech. Engrg., 268:40-64, 2014.

[41] L. Li-Hua and Y. Forterre. Drop impact of yield-stress fluids. Journal of Fluid Mechanics, 632:301-327, 2009.

[42] R. Loubère, P.-H. Maire, and B. Rebourcet. Chapter 13 - staggered and colocated finite volume schemes for Lagrangian hydrodynamics. In Rémi Abgrall and Chi-Wang Shu, editors, Handbook of Numerical Methods for Hyperbolic ProblemsBasic and Fundamental Issues, volume 17 of Handbook of Numerical Analysis, pages 319 - 352. Elsevier, 2016.

[43] R. Loubère, P.-H. Maire, and P. Váchal. 3D staggered Lagrangian hydrodynamics scheme with cell-centered Riemann solver-based artificial viscosity. International Journal for Numerical Methods in Fluids, 72:22 - 42, 2013.

[44] P.-H. Maire. A high-order cell-centered Lagrangian scheme for two-dimensional compressible fluid flows on unstructured meshes. Journal of Computational Physics, 228:2391-2425, 2009. 
[45] P.-H. Maire. A unified sub-cell force-based discretization for cell-centered Lagrangian hydrodynamics on polygonal grids. Int. J. Numer. Meth. Fluid, 65:1281-1294, 2011.

[46] P.-H. Maire. Contribution to the numerical modeling of Inertial Confinement Fusion. PhD thesis, University of Bordeaux, France, 2011. Habilitation à diriger des recherches, available at https://www.researchgate.net/publication/304111873_Habilitation_Defense_ Contribution_to_the_Numerical_Modeling_of_Inertial_Confinement_Fusion.

[47] P.-H. Maire, R. Abgrall, J. Breil, R. Loubère, and B. Rebourcet. A nominally secondorder cell-centered Lagrangian scheme for simulating elastic-plastic flows on two-dimensional unstructured grids. J. Comput. Phys., 235(C):626-665, February 2013.

[48] D. J. Payen and K.-J. Bathe. A stress improvement procedure. Computers \& Structures, 112-113:311-326, 2012.

[49] I. Peshkov, W. Boscheri, R. Loubère, E. Romenski, and M. Dumbser. Theoretical and numerical comparison of hyperelastic and hypoelastic formulations for Eulerian non-linear elastoplasticity. J. Comput. Phys., 387:481 - 521, 2019.

[50] B. Plohr and J. Plohr. Large deformation constitutive laws for isotropic thermoelastic materials. Technical Report LA-UR-12-23451, Los Alamos National Laboratory, 2012.

[51] J. Massoni S. Hank, N. Favrie. Modeling hyperelasticity in non-equilibrium multiphase flows. Journal of Computational Physics, 330:65-91, 2017.

[52] S.K. Sambasivan, M.J. Shashkov, and D.E. Burton. A finite volume cell-centered Lagrangian hydrodynamics approach for solids in general unstructured grids. Int. J. Numer. Meth. Fluid, 72:770-810, 2013.

[53] G. Scovazzi, B. Carnes, X. Zeng, and S. Rossi. A simple, stable, and accurate linear tetrahedral finite element for transient, nearly, and fully incompressible solid dynamics: a dynamic variational multiscale approach. International Journal for Numerical Methods in Engineering, 106:799-839, 2016.

[54] V.A. Titarev and E.F. Toro. ADER schemes for three-dimensional nonlinear hyperbolic systems. Isaac Newton Institute for Mathematical Sciences Preprint Series, 2003.

[55] J. A. Trangenstein. A second-order Godunov algorithm for two-dimensional solid mechanics. Computational Mechanics, 13(5):343-359, September 1994.

[56] J.A. Trangenstein and P. Colella. A higher-order Godunov method for modeling finite deformation in elastic-plastic solids. Communications on Pure and Applied Mathematics, 44(1):41-100, 1991.

[57] C. Truesdell. Hypo-elasticity. Journal of Rational Mechanics and Analysis, 4:83-1020, 1955.

[58] C. Truesdell. Remarks on hypo-elasticity. Journal of Research of the National Bureau of Standards - B. Mathematics and Mathematical Physics, 67B:141-143, 1963.

[59] M.L. Wilkins. Calculation of elastic plastic flow. In B. Alder, S. Fernbach, and M. Rotenberg, editors, Methods in Computational Physics, volume 3, pages 211-263. Academic Press, New York, 1964. 